\title{
First Semester Calculus Students' Concept Definitions and Concept Images of the Tangent Line and How These Relate to Students' Understandings of the Derivative
}

\author{
Brittany Vincent
}

Follow this and additional works at: https://researchrepository.wvu.edu/etd

\author{
Recommended Citation \\ Vincent, Brittany, "First Semester Calculus Students' Concept Definitions and Concept Images of the \\ Tangent Line and How These Relate to Students' Understandings of the Derivative" (2016). Graduate \\ Theses, Dissertations, and Problem Reports. 6876. \\ https://researchrepository.wvu.edu/etd/6876
}

This Dissertation is protected by copyright and/or related rights. It has been brought to you by the The Research Repository @ WVU with permission from the rights-holder(s). You are free to use this Dissertation in any way that is permitted by the copyright and related rights legislation that applies to your use. For other uses you must obtain permission from the rights-holder(s) directly, unless additional rights are indicated by a Creative Commons license in the record and/ or on the work itself. This Dissertation has been accepted for inclusion in WVU Graduate Theses, Dissertations, and Problem Reports collection by an authorized administrator of The Research Repository @ WVU.

For more information, please contact researchrepository@mail.wvu.edu. 
First Semester Calculus Students' Concept Definitions and Concept Images of the Tangent Line and How These Relate to Students' Understandings of the Derivative

\title{
Brittany Vincent
}

\author{
Dissertation submitted \\ to the Eberly College of Arts and Sciences \\ at West Virginia University \\ in partial fulfillment of the requirements for the degree of
}

Doctor of Philosophy in

Mathematics

\author{
Vicki Sealey, Ph.D., Chair \\ Nicole Engelke Infante, Ph.D. \\ Marjorie Darrah, Ph.D. \\ Harvey Diamond, Ph.D. \\ Scott Myers, Ph.D.
}

Department of Mathematics

\author{
Morgantown, West Virginia \\ 2016
}

Keywords: tangent line, derivative, calculus, concept definition, concept image Copyright 2016 Brittany Vincent 


\section{ABSTRACT \\ First Semester Calculus Students' Concept Definitions and Concept Images of the Tangent Line and How These Relate to Students' Understandings of the Derivative Brittany Vincent}

The purpose of this dissertation is to examine how students define and think about the tangent line in first semester calculus and investigate the influence of these ways of thinking on their understandings of the derivative. Students' conceptions of the tangent line were explored through four primary tasks: defining and constructing tangent lines, sketching the derivative, and graphically interpreting the formal symbolic definition of the derivative. The first two tasks were designed to access students' knowledge of tangent lines, and the second two tasks drew upon their ability to apply this knowledge and connect the tangent line to the derivative. In this dissertation, I describe students' responses in terms of overlap or lack of overlap with how the tangent line and derivative are formally defined. The Tangent Line Framework of this dissertation and the Derivative Framework developed by Zandieh (2000) were used to structure this knowledge. The frameworks present a diagrammatic way to illustrate the understandings evidenced by the students and graphically contrast these for the concepts of tangent line and derivative. The results of this analysis revealed ways in which students' concept images of the tangent line and derivative relate. 


\section{DEDICATION}

This dissertation is dedicated

to my sweet daughters,

Charlotte Love and Mary Grace, and to my Nan. 


\section{ACKNOWLEDGEMENTS}

It would not have been possible to write this dissertation without the help and guidance of the kind people around me, to only some of whom it is possible to give particular mention here.

I am grateful to my advisor, Dr. Vicki Sealey, for supporting this project and giving such thoughtful feedback. I have appreciated her kind way of redirecting me and the encouragement she has offered along the way. Her expertise, insight, and generous guidance have been paramount to the completion of this dissertation.

I would also like to thank the additional members of my dissertation committee, Dr. Marjorie Darrah, Dr. Harvey Diamond, Dr. Nicole Engelke, and Dr. Scott Myers. Thank you for your contributions to this project and for serving on this committee. My deepest appreciations.

To my husband, Patrick, thank you for being my comic relief. Thank you for your emotional support and great patience at all times. You've done a great job entertaining the girls while I have worked, and I thank you for the willingness to do whatever was needed in order for me to finish this dissertation. I am looking forward to joining the fun again! I am thankful for you and love you very much.

A special thank you to my mom, Marcia, who has been a source of encouragement and inspiration to me throughout my life. Thank you for providing me a "writing space" and for nurturing me through the months of writing. Because of you, the difficult days were sprinkled with laughter and kindness and love. Thank you for making yourself endlessly available to help watch the kids and for putting your life on pause as I finished this dissertation. You and Sam bringing me coffee at the library was worth more than gold.

To my sister and best friend, Megan, thank you. You have influenced and encouraged me in so many ways over the years. I could not imagine a better sister than you. My brothers, Luke and Tyler, thank you for the support. Ty, I have enjoyed your company at the library and will miss seeing you there, but will be happy to buy you Starbucks outside of our school work anytime. I would also like to thank my mother-in-law, Pam, who has been a big help over the years as I have worked to complete this dissertation.

To my favorite little girls in the whole world, Charlotte and Mary, thanks for all the play breaks! You girls make my heart so full. Charlotte, thanks for the presents you have made me over the past months. You're the best gift-giver. Mary, your silly little smile has helped keep my burden light. I love you girls.

My friends from WVU, Renee, Kristy, Janet, Celeste, Todd, Will, and many others, I am so glad to have met such a unique group of individuals, and because of you all, I have very fond memories of graduate school. To all the mathematics instructors I have had over the years, both FSU and WVU, thank you for instilling in me a passion for analytic thinking and love of learning.

Thank you to all my family and friends who have been a source of encouragement, grace, and love, I am deeply humbled by all of your support. A hundred times over, thank you.

I would like to thank God, through whom I live, and move, and have my being. 


\section{TABLE OF CONTENTS}

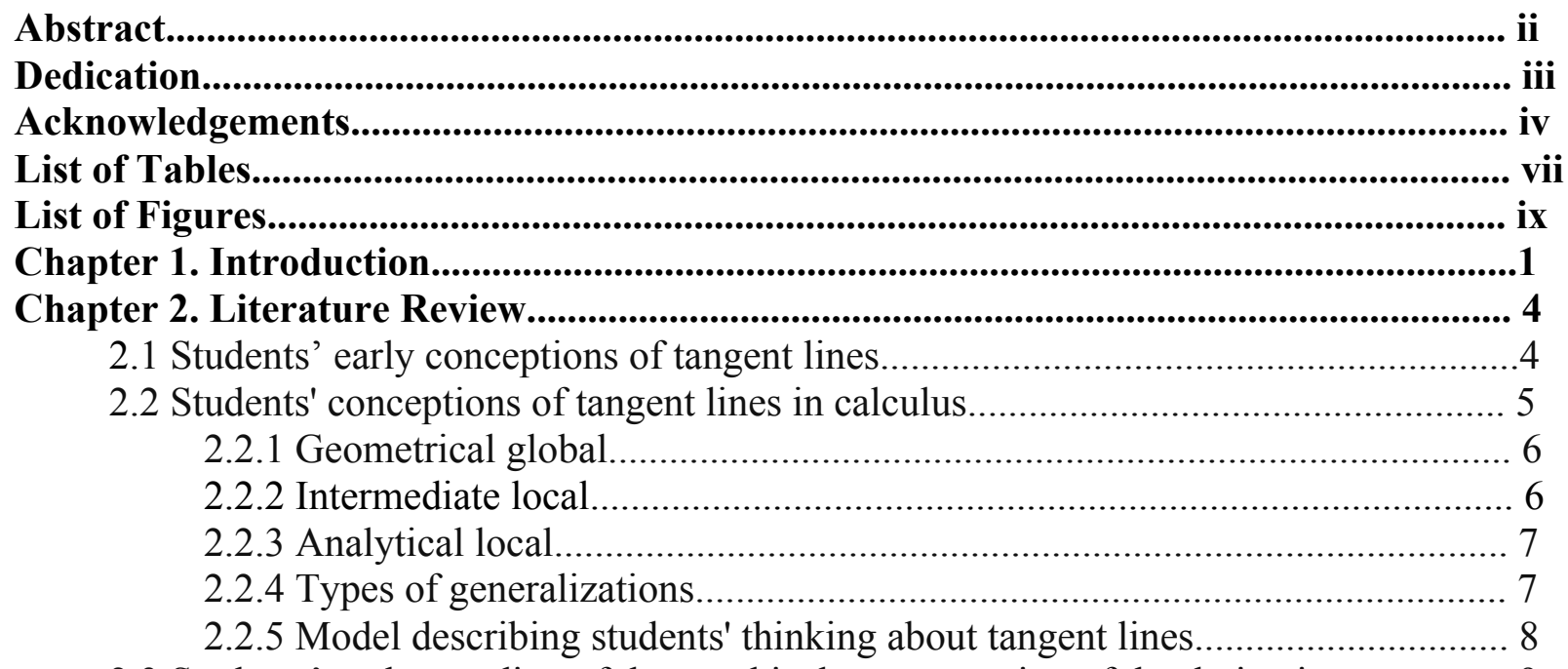

2.3 Students' understanding of the graphical representation of the derivative................ 9

2.4 Role of mathematical definitions................................................................... 12

2.4.1 Meaning students' associate with mathematical definitions.......................... 12

2.4.2 A framework that structures the role of defining.......................................... 14

Chapter 3. Theoretical Perspective................................................................................................... 17

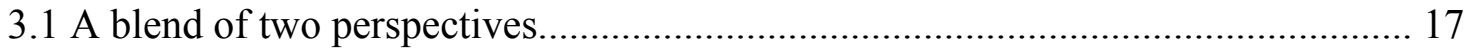

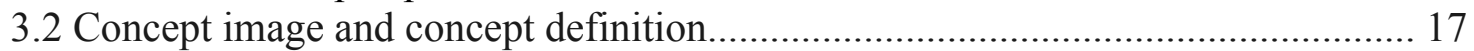

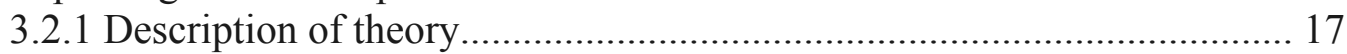

3.2.2 Concept formation and different thought processes................................ 20

3.3 Zandieh's Derivative Framework..................................................................... 24

3.3.1 Layers of process-object pairs............................................................ 25

3.3.2 Sfard's theory of processes and objects.................................................... 29

3.3.3 Tangent Line Framework..................................................................... 30

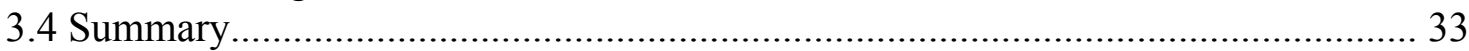

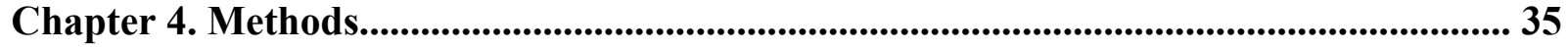

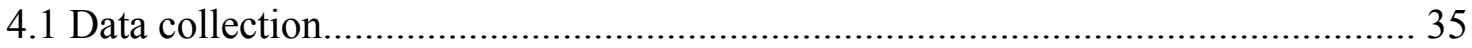

4.1.1 Pilot studies...................................................................................... 35

4.1.2 Overview of dissertation study ................................................................ 36

4.1.3 Sample selection and participant demographics..................................... 36

4.1.4 Interview process.................................................................................. 39

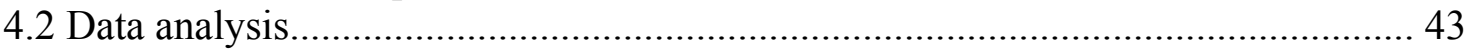

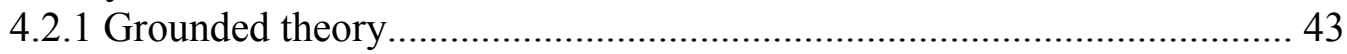

4.2.2 Phase 1- open and axial coding............................................................. 44

4.2.3 Phase 2- selective coding.................................................................... 48

4.2.4 Phase 3- theorizing............................................................................ 52

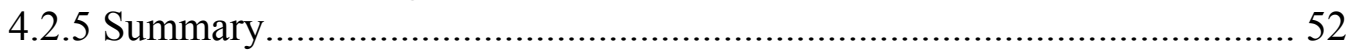

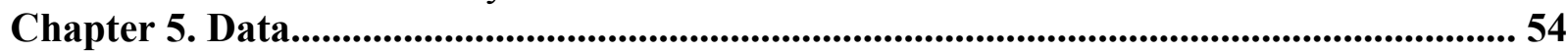

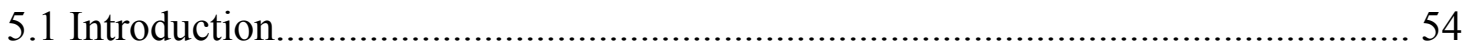

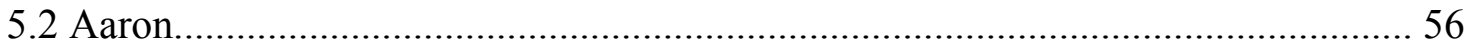

5.2.1 Task 1: Concept definition of the tangent line........................................ 56

5.2.2 Task 2: Construction of tangent lines- six graphs...................................... 57 
5.2.3 Task 3: Sketching the graph of $f^{\prime}(x)$, given the graph of $f(x) \ldots \ldots \ldots \ldots . . . .60$

5.2.4 Task 4: Graphically interpreting the formal symbolic definition

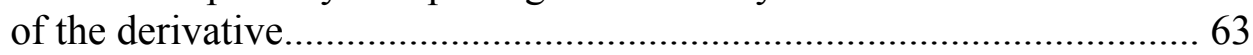

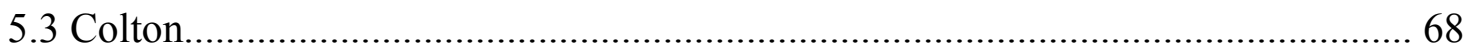

5.3.1 Task 1: Concept definition of the tangent line.......................................... 68

5.3.2 Task 2: Construction of tangent lines- six graphs..................................... 68

5.3.3 Task 3: Sketching the graph of $f^{\prime}(x)$, given the graph of $f(x) \ldots \ldots \ldots \ldots \ldots . .74$

5.3.4 Task 4: Graphically interpreting the formal symbolic definition

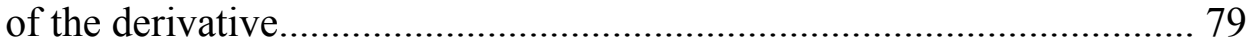

5.4 Jamie 85

5.4.1 Task 1: Concept definition of the tangent line..................................... 85

5.4.2 Task 2: Construction of tangent lines- six graphs................................ 87

5.4.3 Task 3: Sketching the graph of $f^{\prime}(x)$, given the graph of $f(x) \ldots \ldots \ldots \ldots . . . .90$

5.4.4 Task 4: Graphically interpreting the formal symbolic definition

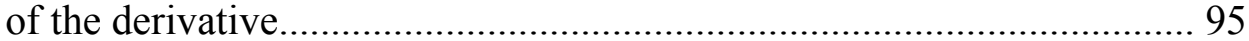

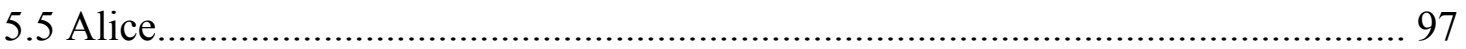

5.5.1 Task 1: Concept definition of the tangent line..................................... 97

5.5.2 Task 2: Construction of tangent lines- six graphs................................. 98

5.5.3 Task 3: Sketching the graph of $f^{\prime}(x)$, given the graph of $f(x) \ldots \ldots \ldots \ldots . . . .102$

5.5.4 Task 4: Graphically interpreting the formal symbolic definition

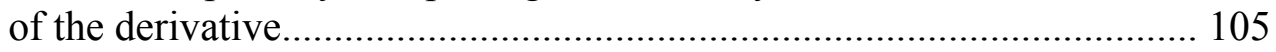

5.6 Tangent Line Framework...................................................................................... 108

Chapter 6. Results............................................................................................................................ 111

6.1 Introduction........................................................................................................ 111

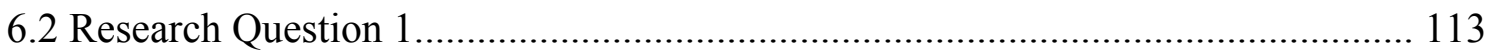

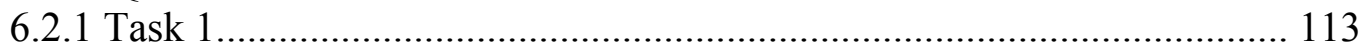

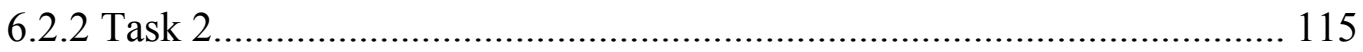

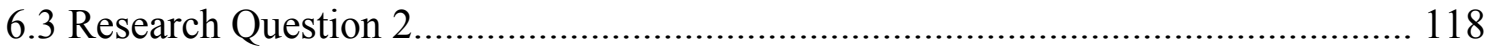

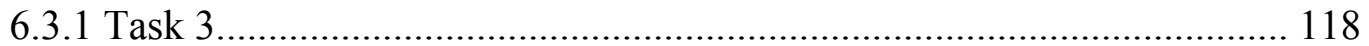

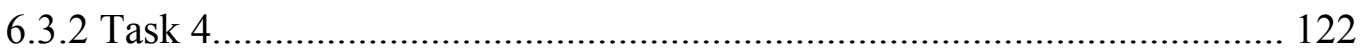

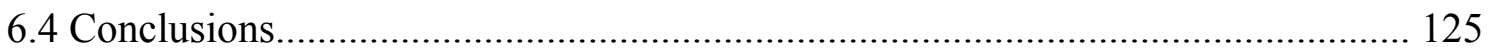

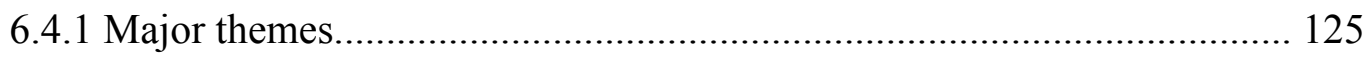

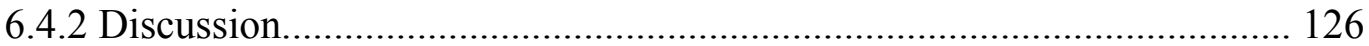

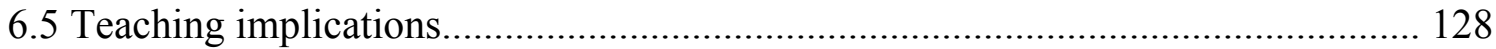

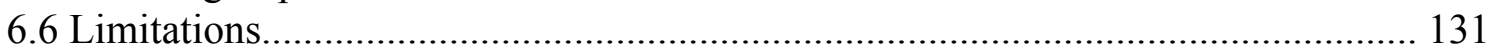

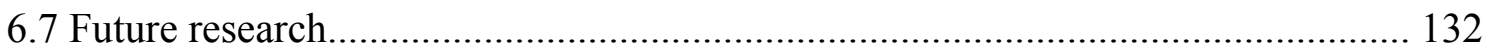

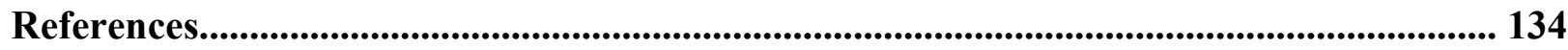

Appendix A. Questionnaire...................................................................................................... 137

Appendix B. Interview 1...................................................................................................... 140

Appendix C. Interview 2............................................................................................................. 142

Appendix D. Interview 3..................................................................................................... 143 


\section{LIST OF TABLES}

Table 1. Seven influential factors on students' thinking about tangent lines....................... 8

Table 2. Influential factors for the three perspectives on tangency.................................... 9

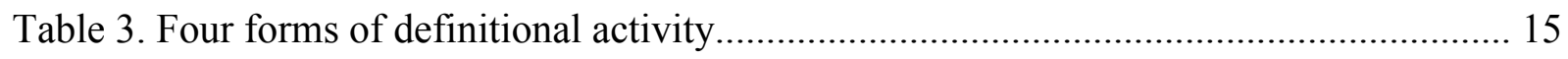

Table 4. Zandieh's three-layer Derivative Framework...................................................... 26

Table 5. Tangent Line Framework, subset of Zandieh's Derivative Framework.................... 31

Table 6. Layers of process-object pairs for the Tangent Line Framework........................... 32

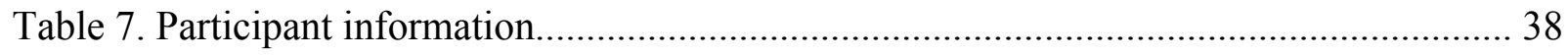

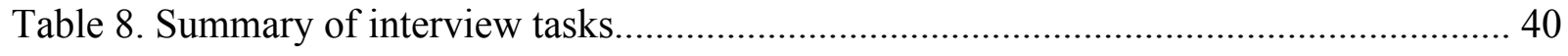

Table 9. Average interview ratings and four core students............................................ 49

Table 10. Tangent Line and Derivative Frameworks............................................... 50

Table 11. Data analysis. Categories, subcategories, and properties................................... 52

Table 12. Aaron. Task 1. Summary chart..................................................................... 56

Table 13. Aaron. Task 2, Interview 3. Summary chart................................................ 58

Table 14. Aaron. Tasks 1 and 2, Interview 3. Concept definition and image of tangent line... 60

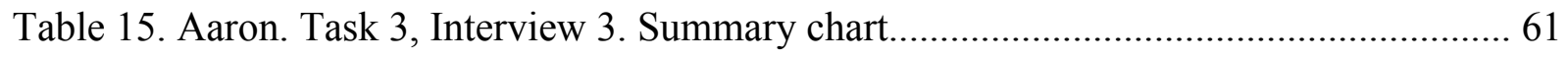

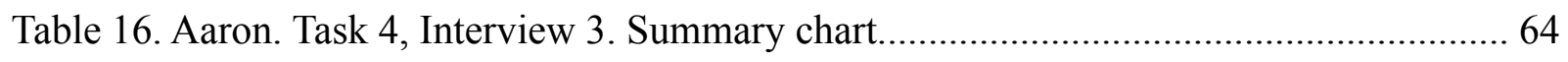

Table 17. Aaron. Tasks 3 and 4, Interview 3. Concept image of the derivative...................... 66

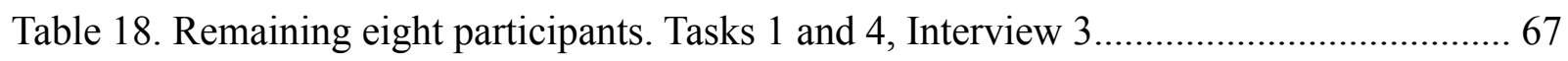

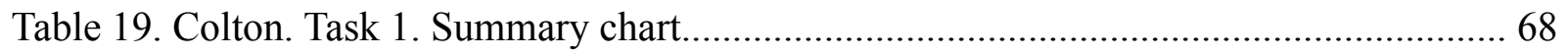

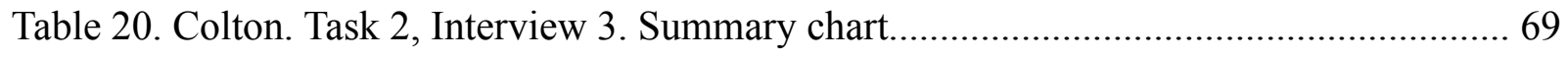

Table 21. Colton. Tasks 1 and 2, Interview 3. Concept definition and image of tangent line... 74

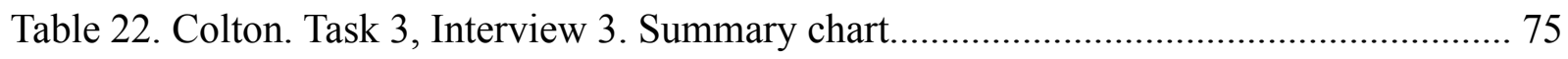


Table 23. Colton. Task 4, Interview 3. Summary chart......................................................... 83

Table 24. Colton. Tasks 3 and 4, Interview 3. Concept image of the derivative....................... 84

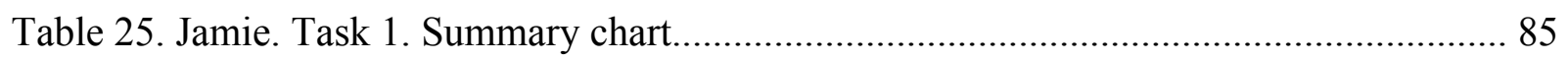

Table 26. Jamie. Task 2, Interview 3. Summary chart.......................................................... 87

Table 27. Jamie. Tasks 1 and 2, Interview 3. Concept definition and image of tangent line.... 90

Table 28. Jamie. Task 3, Interview 3. Summary chart........................................................... 91

Table 29. Jamie. Task 4, Interview 3. Summary chart........................................................... 96

Table 30. Jamie. Tasks 3 and 4, Interview 3. Concept image of the derivative........................ 96

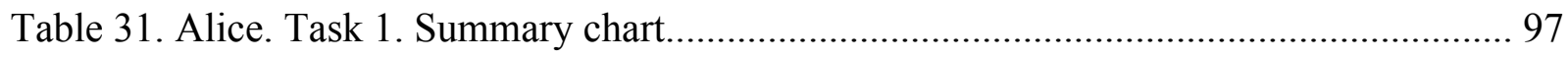

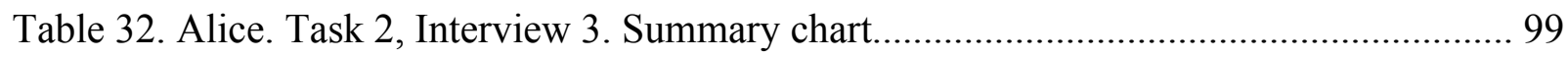

Table 33. Alice. Tasks 1 and 2, Interview 3. Concept definition and image of tangent line.... 102

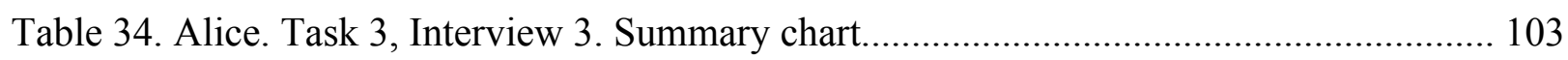

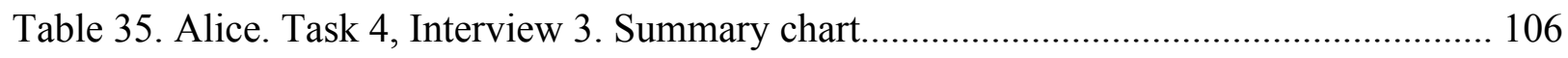

Table 36. Alice. Tasks 3 and 4, Interview 3. Concept image of the derivative......................... 107

Table 37. Tangent Line Framework. Four core students........................................................ 109

Table 38. Tangent Line Framework. Remaining eight participants......................................... 110

Table 39. All twelve participants' concept definitions. Tangent Line Framework................... 113

Table 40. Concept definition, tangent line. Interviews 1-3, Task 1. Four core students........... 116

Table 41. Concept image, tangent line. Interview 3, Task 2. Four core students...................... 116

Table 42. Interview 3, Task 3. Summary chart. Four core students........................................ 119

Table 43. Tasks 1 and 4, Interview 3. Four core students..................................................... 123

Table 44. Tasks 1 and 4. Tangent Line Framework. Four core students.................................. 124

Table 45. Average interview rating compared to previous calculus experience............... 129 


\section{LIST OF FIGURES}

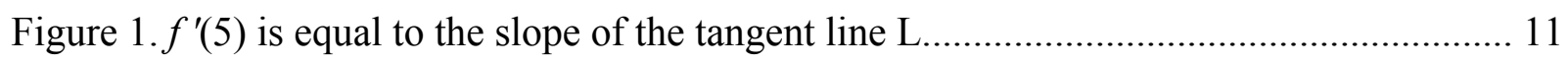

Figure 2. CD and CI inform each other..................................................................... 22

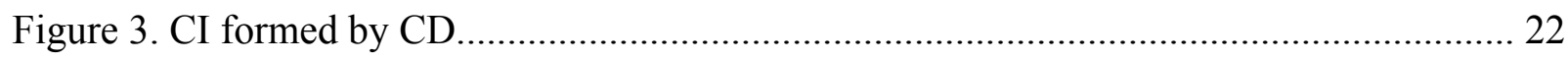

Figure 4. Interplay between definition and image.................................................... 23

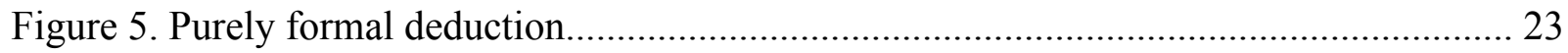

Figure 6. Deduction following intuitive thought........................................................ 23

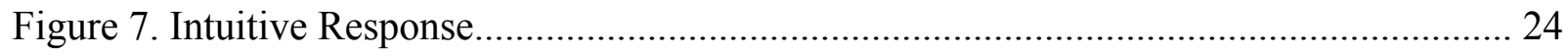

Figure 8. Tangent Line Framework. Chain of process-object pairs................................... 33

Figure 9. StudioCode- movie package and code window............................................... 53

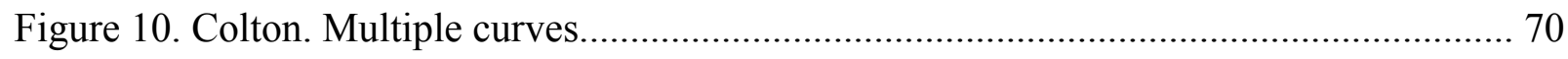

Figure 11. Jamie. Derivative does not exist but tangent line does..................................... 93

Figure 12. Two different thought processes............................................................. 121 


\section{Chapter 1 - Introduction}

The original premise of this dissertation study was to examine how first semester calculus students' understandings of the tangent line transform over the course of a semester. My interest in students' conceptual changes for the tangent line was sparked by contrasting results for two pilot studies. Students of the second pilot study performed significantly better on tangent line construction tasks than their peers in the first pilot study. The variance in results was hypothesized to be the timing of the studies. We hypothesized that students' understandings of the tangent line dim over the course of a semester as they encounter new ideas and work to organize and interpret this information. In order to examine students' understandings of the tangent line over time, my dissertation study included a series of three interviews- one at the beginning, middle, and end of a first semester calculus course. These data did not support the hypothesis of conceptual change suggested by the two pilot studies. Instead, students' scant definitions of the tangent line and the effect of these on their larger understandings of tangency and the derivative was an emergent theme in the data. Accordingly, this dissertation study is motivated by the following questions:

Research Question 1: What are first-semester calculus students' concept definitions and concept images of the tangent line? How do these relate to one another and compare to the formal definition of the tangent line at the first-semester calculus level?

Research Question 2: How do first semester calculus students connect the concepts of the tangent line and the derivative in a graphical representation?

Existing research has identified various student perspectives on tangency (Biza, Christou, \& Zachariades, 2008; Vincent, LaRue, Sealey, \& Engelke, 2015). These studies highlight students' misconceptions about the tangent line, such as it having only one point in common with 
the curve, or it not crossing through the curve at the point of tangency. Existing research has also noted students' difficulties with the graphical representation of the derivative. For example, equating the tangent line at a point with the derivative at that point (as opposed to the slope of the tangent line) or confusing the $y$-coordinate of the point of tangency with the derivative (Amit \& Vinner, 1990; Orton 1983). This dissertation seeks to bridge the gap between these two areas of research by analyzing students' understandings of the tangent line and the derivative using parallel frameworks, and then, examining the role of tangent lines on students' mental blockages associated with the derivative.

Twelve first-semester calculus students enrolled in West Virginia University during the spring 2015 semester took part in this dissertation study. Each student completed a questionnaire and a series of three interviews spaced over the course of the semester. Collectively, the interview tasks included defining a tangent line (Task 1), constructing tangent lines (Task 2), sketching the derivative of a function for which the algebraic representation was unknown (Task 3), and graphically interpreting the formal symbolic definition of the derivative (Task 4). The first two tasks examined students' basic understandings of tangent lines, and the second two tasks explored students' abilities to apply their knowledge of tangent lines in the context of the derivative.

The data was analyzed using a blend of theoretical perspectives. Tall and Vinner's (1981) notions of concept image and concept definition encompass the understandings displayed by the students. The term concept definition is defined to be "a form of words used to specify that concept," and a concept image is regarded as "the total cognitive structure that is associated with the concept, which includes all the mental pictures and associated properties and processes" (Tall \& Vinner, 1981, p. 152). My research describes these internal structures for the concepts of the 
tangent line and the derivative in terms of overlap or lack of overlap with how the concepts are formally defined. These formal definitions are represented by Zandieh's (2000) Derivative Framework and the Tangent Line Framework of this paper.

Findings showed that phrases used by students to define the tangent line were indicative of what they most strongly associated with the concept. In other words, their concept definitions represented the prioritized elements of their concept images. This was problematic given the inaccuracy of their definitions. These insufficient definitions manifested in different ways as students solved problems related to the derivative, such as an inability to geometrically define the derivative as the slope of the tangent line or to connect the limit in the formal definition of the derivative to its graphical meaning.

In general, the participants seemed to form their definitions of the tangent line on the spot, in response to the interview questions. Most participants associated little meaning with their imprecise definitions, and therefore, demonstrated concept-image based ways of reasoning about the tangent line. These patterns of thought, which did not consult definitions, prevented students from connecting the tangent line and the derivative in a meaningful way. Results suggest that calculus instruction should encourage students to reflect on their definitions of tangency, and care should be taken to model concept-definition based reasoning in the classroom. 


\section{Chapter 2 - Literature Review}

\subsection{Students' early conceptions of tangent lines}

Students encounter tangent lines in Euclidean Geometry as tangent to a circle, in Analytic Geometry as tangent of conic sections, and in Analysis as tangent to a function graph (Biza et al., 2008). Their early experiences with tangents in geometry may result in tacit theories about what tangents are, which can influence the way they think about tangents in subsequent settings (Fischbein, 1987). For example, when constructing the tangent to a function graph in calculus, students may apply geometric properties: it keeps the curve in the same semi-plane, it may only meet the curve at one point, and it may not cross the curve at that point (Biza et al., 2008). Thus, students are interpreting properties of tangent lines from Geometry as defining conditions for the more general case of the tangent line to a function graph (Winicki \& Leikin, 2000).

The tangent to a circle and the tangent to a function graph both involve the notion of tangent, but the definition of tangent varies across the different curricular contexts. The notion of tangent line in geometry is characterized by global relations (e.g., a tangent line is the line that has only one point in common with the circle), whereas tangency in analysis is characterized by a local perspective (e.g., the tangent line through point $\mathrm{A}$ is the limiting position of secant lines AB). The two perspectives are epistemologically different, which requires students to reconstruct their definition of tangent as they progress through their mathematical studies. Some students will reconstruct their concept image of tangent, others may just apply geometric properties locally at the point of tangency, and some may hold on to geometric properties of tangent lines and apply them globally to a function graph (Biza et al., 2008).

With all of their past experiences, students' concept image of tangent line becomes very complex (Tall, 1987). They are building their concept image of tangent line as they meet it in 
new contexts. As they encounter various cases, such as the tangent line at an inflection point, tangent lines that coincide with the graph or cross over the graph, or places where the tangent line does not exist, their confusion about the general meaning of a tangent line to a function graph becomes evident (Biza et al., 2008).

The various definitions of tangent line used across different contexts may contribute to students' confusion about tangent lines in calculus. Additionally, students' confusions about the tangent line may be attributed to the "difficulties they often have with the generalization of the definition of a concept, particularly when they have already encountered specific cases of the same concept" (Biza, 2011, p. 128). This generalization requires a student to reconsider all previously met specific cases in the light of the general definition. Consequently, their understanding of the tangent line will need to be restructured to align with the formal definition of the concept.

Since my research seeks to explore how students' concept definitions of the tangent line and their understanding of the derivative are related, the remainder of this chapter focuses on three areas of research:

1. Students' conceptions of tangent lines in calculus.

2. Students understanding of the graphical representation of the derivative.

3. The role of mathematical definitions

\subsection{Students' conceptions of tangent lines in calculus}

The above literature demonstrated the influence of students' initial conceptualizations of a tangent line within a given context (e.g., Euclidean Geometry) on their understanding in subsequent settings (e.g. Calculus). The work of Biza et al. (2008) contributes to the above results by putting forth a model that describes how students, who have met the notion of tangent 
line in different contexts, think about tangents. In their research, they put forth three perspectives on tangent lines that students may hold: Analytical Local, Intermediate Local, and Geometrical Global. This section reviews these three perspectives on tangency and outlines six factors that influence how students' think about tangent lines.

\subsubsection{Geometrical global}

A geometrical global perspective is characterized by a definition of the tangent line as a line that touches the graph only once and does not cross over it. Essentially, students in this group apply the geometrical properties of the tangent line globally to a function graph. Their understandings are influenced by the properties of a tangent line to a circle from geometry, and they tend to think of the tangent line in calculus as having only one common point with the graph. Students with this perspective may reason about tangent lines by looking at properties of the whole graph rather than looking at a neighborhood of the common point. Because of this, they often reject tangent lines at inflection points. They also incorrectly accept tangent lines at corner points.

\subsubsection{Intermediate local}

An intermediate local perspective is characterized by applying the geometrical properties of tangent lines locally at the point of tangency. For example, the tangent line may have only one point in common with the curve locally and must stay in the same semi-plane as the curve locally (cannot cross through the curve at the point of tangency). Unlike the geometrical global group, students with an intermediate local perspective would accept a tangent line that had more than one point in common with the curve- as long as this other point was not in a neighborhood of the tangency point. By applying geometrical properties locally, students may reject tangent lines that coincide with the curve near the point of tangency. They may also reject the tangent line at an 
inflection point, since locally the curve does not remain in the same semi-plane as the tangent line. Students classified as intermediate local are more likely to not accept tangent lines at corner points compared to the geometrical global group. Essentially, students in this group have elaborated the properties of the circle tangent (e.g. one common point or remaining in the same semi-plane) in order to apply them in a neighborhood of the point of tangency. "These elaborated images of the tangent remain inadequate for the general case of the function graph, as they do not include cases such as the tangent line at an inflection point or the coincidence of the tangent and the curve" (Biza et al., 2008, p. 63).

\subsubsection{Analytical local}

In general, students with an analytical local perspective on tangency have a more sophisticated concept image of tangent lines. Their concept images typically involve cases for which the tangent line has more than one common point with the curve, intersects with the curve, and coincides with the curve. However, students in this group are more likely to accept tangent lines at corner points compared to students with an intermediate local perspective. Biza et al. (2008) reported this may be due to the fact that students in this group consider the limiting position of secant lines from both the left and right side of the point of tangency. This sometimes resulted in them having two tangent lines constructed at a corner point. Overall, students classified as analytical local demonstrate a concept image built on the local approach to tangency that characterizes its use in Analysis.

\subsubsection{Types of generalizations}

These three different perspectives on tangency may be described using two types of generalization outlined by Harel and Tall (1991). Expansive generalization is an expansion of a students' existing cognitive structure without requiring changes in their current schemes. 
Reconstructive generalization requires a radical change in their old concept image, so that the concept can be more broadly applied. Reconstructive generalization is required for students to transition from a global to a local perspective of tangency, especially in the case of the general function graph (Biza et al., 2008). Students in the intermediate local group expanded their concept image of tangent by applying global properties locally at the point of tangency, whereas students in the analytical local group reconstructed their concept image of the tangent line. On the other hand, the geometrical global group neither expanded nor reconstructed their concept images, but instead, persisted in applying geometric properties of the tangent line to the general case of a function graph. Acquisition of the general meaning of tangency requires students to reconstruct their mathematical knowledge of this concept (Biza et al., 2008).

\subsubsection{Model describing students' thinking about tangent lines}

As a result of their study, Biza et al. (2008) identified seven factors that are influential on students' thinking about tangent lines (F1-F7). Using Confirmatory Factor Analysis, they confirmed that six of these factors (F2-F7) constitute a model describing students thinking about tangent lines (Table 1).

\begin{tabular}{|c|l|}
\hline Factors & Description \\
\hline F1 & The tangent line could have only one common point with the curve. \\
\hline F2 & $\begin{array}{l}\text { The tangent line could have only one common point in a neighborhood of the } \\
\text { tangency point. }\end{array}$ \\
\hline F3 & $\begin{array}{l}\text { In any neighborhood of the tangency point the tangent line could have an } \\
\text { infinite number of common points with the curve. }\end{array}$ \\
\hline F4 & There exists a tangent line at an inflection point. \\
\hline F5 & There is no tangent line at an edge point. \\
\hline F6 & Symbolic manipulation of the tangent line. \\
\hline F7 & Tangent of conic sections. \\
\hline
\end{tabular}

Table 1. Seven influential factors on students' thinking about tangent lines. 
By associating the factors shown in Table 1 with the students' performance on the questionnaire, they deduced that the analytical local group performed satisfactorily in terms of all factors; the intermediate local group in terms of factors F1, F2, F5, F6, and F7; and the geometrical global group in terms of factors F1 and F7. Table 2 below demonstrates, for each perspective, their dominant concept images as well as corresponding influential factors (Biza et al., 2008).

\begin{tabular}{|l|l|l|}
\hline Perspective & Dominant Concept Image & Factors \\
\hline $\begin{array}{l}\text { Analytical } \\
\text { Local }\end{array}$ & $\begin{array}{l}\text { The tangent line: } \\
\text { could have more than one point with the curve; exists at an inflection } \\
\text { point; could coincide with the curve; and does not exist at an edge point. } \\
\text { Satisfactory performance in symbolic manipulation. }\end{array}$ & $\begin{array}{l}\text { F1, F2, F3, } \\
\text { F4, F5, F6, } \\
\text { F7 }\end{array}$ \\
\hline $\begin{array}{l}\text { Intermediate } \\
\text { Local }\end{array}$ & $\begin{array}{l}\text { The tangent line: } \\
\text { could have more than one common point with the curve but there is a } \\
\text { neighbourhood around the tangency point where there is no other common } \\
\text { point between the line and the curve; does not exist at an inflection point; } \\
\text { and, does not exist at an edge point. } \\
\text { Satisfactory performance in symbolic manipulation. }\end{array}$ & F6, F7, F5, \\
\hline $\begin{array}{l}\text { Geometrical } \\
\text { Global }\end{array}$ & $\begin{array}{l}\text { The tangent line: } \\
\text { has only one common point with the curve and leaves the curve in the } \\
\text { same semi-place, or has only one common point with the curve and could } \\
\text { exist at an inflection point; could exist at an edge point. } \\
\text { Unsatisfactory performance in symbolic manipulation. }\end{array}$ & F1, F7 \\
\hline
\end{tabular}

Table 2. Influential factors for the three perspectives on tangency.

\subsection{Students' understanding of the graphical representation of the derivative}

The formal definition of the derivative is given symbolically as the limit of the difference quotient (LDQ), $f^{\prime}(x)=\lim _{h \rightarrow 0} \frac{f(x+h)-f(x)}{h}$. It is possible for students to successfully use LDQ or apply derivative rules without understanding the graphical connection. Skills in visualization are critical for maiking these graphical connections. In fact, Zimmerman (1991) considered skills in visualization to be the core of the calculus reform movement: "Conceptually, the role of visual thinking is so fundamental to the understanding of calculus that it is difficult to imagine a 
successful calculus course which does not emphasize the visual elements of the subject" (p. 136). Tangent lines play a key role in the visual aspect of the derivative concept.

Emphasizing the visual aspects of calculus concepts differs from the traditional belief that doing calculus is equivalent to acquiring skills in the manipulation of symbols. Hahkioniemi (2006) points out that $\lim _{x \rightarrow a} \frac{x^{2}-a^{2}}{x-a}=\lim _{x \rightarrow a} x+a=2 a$ can be solved by manipulating symbols without considering a limiting process, and that even the limit expression, $\lim _{x \rightarrow a}$, could be left out, as some participants in his study did, and still be solved successfully. Research has demonstrated that, in general, students' understandings are typically algebraic and not visual (e.g., Habre \& Abboud 2006, Asiala, Cottrill, Dubinsky, \& Schwingendorf, 1997).

In studying students' graphical understanding of the derivative concept, Asiala et al. (1997) provided participants with the graph shown in Figure 1 on the next page and asked them to determine $f^{\prime}(5)$. The authors noted that an "overpowering idea" among some of the participants was the need for an algebraic expression that could be differentiated (p. 415). Other students found $f^{\prime}(5)$ by computing the equation of the tangent line (finding its slope in the process) and then differentiating this expression! Others confused the equation of the tangent line with that of the original function, and in some cases, with the derivative of the function. These results demonstrate students' preferences toward algebraic techniques. They also evidence a general lack of knowledge on the part of students concerning the role of tangent line for the concept of derivative. 


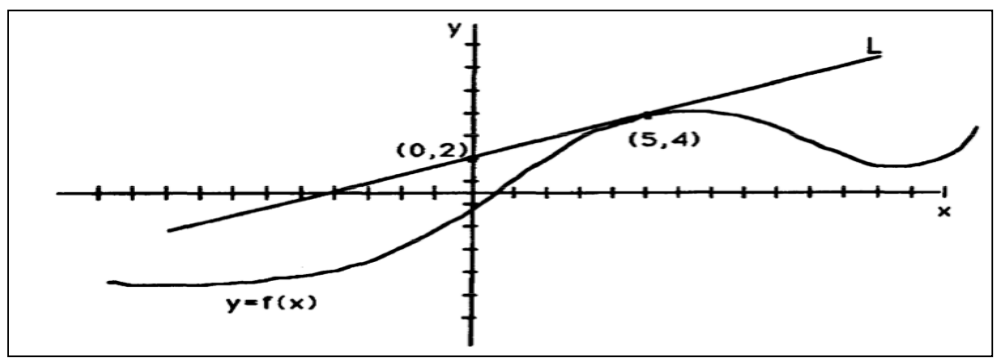

Figure 1. $f^{\prime}(5)$ is equal to the slope of the tangent line L.

Similar results were found in the research of Habre and Abboud (2006). The participants (89 Calculus I students) were given the graph of a parabola (resembling $f(x)=x^{2}$ ) and were asked to deduce whether its derivative would be increasing or decreasing. Fifteen percent of the students stated that a formula must be assigned to the graph in order to make this conclusion. When asked to provide a geometric definition of the derivative, $47.5 \%$ were unsuccessful in their definition (Habre \& Abboud, 2006). The same group was asked to explain geometrically why both $f(x)=x^{2}$ and $g(x)=x^{2}+1$ have a derivative of $2 x$. The expected response was for students to observe that $g(x)$ is a vertical translation of $f(x)$, and therefore, the tangent lines at a given point remain parallel; consequently, the derivatives are equal. Results showed that only about $13 \%$ of the students gave the desired justification (Habre \& Abboud, 2006).

Given the graph of a function, students may struggle to determine the value of the derivative at a given $x$-value by reasoning in terms of the slope of the tangent line. Research has shown that students also have difficulty interpreting what the formal definition of the derivative means graphically. In Hahkioniemi's (2006) study with five eleventh grade differential calculus students, only one of the participants could interpret the difference quotient from the definition of the derivative as the slope of a secant line. Two of the five could not draw tangent lines. Zandieh (2000) explained that a student may understand LDQ as a pseudo-object without having knowledge of the underlying limiting process. Students can be very successful at applying 
derivative rules or solving problems involving instantaneous rate of change without understanding the underlying processes of the derivative concept. It is important to consider how students' misconceptions concerning the tangent line contribute to their lack of conceptual understanding of the derivative. This is a primary goal of this dissertation study.

Overall, these results indicate that students' struggle with the meaning of tangent lines in relation to the derivative concept. Their preferences toward algebraic techniques reveal a lack of understanding of the graphical meaning of the derivative concept. This dissertation study contributes to these results by illustrating in a diagrammatic way how students' concept definitions of the tangent line translate to their conceptual understandings of the derivative. In particular, the case is made that students' concept definitions of the tangent line have the potential to create mental blockages and impede their ability to develop a robust understanding of the derivative.

\subsection{Role of mathematical definitions}

\subsubsection{Meaning students' associate with mathematical definitions}

Edwards and Ward (2004) conducted interviews with undergraduate mathematics majors enrolled in an abstract algebra course to examine their understandings of the role of formal definitions in mathematics. Their research was influenced by the results of Edwards's dissertation (1997), which examined the same topic but with undergraduate majors enrolled in real analysis. Edwards (1997) found that tasks involving the use of a formal definition were problematic for some students. For example, participants seemed to place higher priority on their memories or previously seen examples than on the formal definition of a concept. Students in her study also demonstrated negative beliefs towards definitions. For example, one student referred to mathematical definitions as a "lot of jargon" and stated: "After about the first day in calculus, we 
didn't care about this [formal definition] ... if you had the concept right, not really the definition, that was all that really mattered" (Edwards \& Ward, 2008, p. 227-228).

Vinner explains, "It seems to us that many teachers at the secondary and the collegiate levels expect a one-way process for the concept formation... they expect that the concept image will be formed by means of the concept definition and will be completely controlled by it" (1991, p. 71). Ward admitted to having similar expectations at the beginning of their study with abstract algebra students (Edward \& Ward, 2004). He believed students difficulties with mathematical definitions in real analysis could be contributed to their flawed concept images formed by prior experience, but in the case of abstract algebra, where students are meeting terms such as 'coset' or 'group' for the first time, he expected different results. He expected, just as Vinner (1991) demonstrated, that students' concept images would be formed and controlled by their concept definitions. This was not the case. The participants did not use the concept definition in the way that "an experienced mathematician would use it" (p. 418). Edwards and Ward (2004) found that even though students were encountering new mathematical notions and definitions, there were preexisting concept images that competed with or may even have replaced the concept definition as the controlling force of the concept image.

Edwards and Ward (2004) suggest that students' difficulties with mathematical definitions do not necessarily arise from the content of the definition; rather their difficulties are related to the very nature of mathematical definitions. Comparing both studies, Edwards's dissertation with real analysis students (1997) and their joint work with abstract algebra students (2004), they identified three "surprises" concerning students' understanding of the role of mathematical definitions as well as three implications for teaching: 
Surprise 1. Many students do not categorize mathematical definitions the way mathematicians do.

Surprise 2. Many students do not use definitions the way mathematicians do, even when the students can correctly state and explain the definitions.

Surprise 3. Many students do not use definitions the way mathematicians do, even in the apparent absence of any other course of action.

Implication 1. The special nature of mathematical definitions should be treated as a concept in its own right, one that should be understood at some level by all college mathematics students.

Implication 2. Transitioning from concept-image-based reasoning to concept-definitionbased reasoning can be difficult and puzzling for many students.

Implication 3. The results of this research should be considered in the mathematical preparation of future teachers.

In light of this, they put forth the concern that, although textbooks and many calculus instructors do not emphasize formal definitions, we must begin to consider the implications of these pedagogical decisions on students' mathematical understandings (Edwards \& Ward, 2008).

\subsubsection{A framework that structures the role of defining}

The defining as a mathematical activity framework (DMA) described in the work of Zandieh and Rasmussen (2010) integrates two lines of research: "the instructional design theory of Realistic Mathematics Education (RME) and distinctions between concept image and concept definition" (p. 57). In short, RME focuses on sequences of tasks that allow students to transition from models-of their mathematical activity to models-for more advanced mathematical reasoning (Zandieh \& Rasmussen, 2010). Zandieh and Rasmussen (2010) demonstrate that, in a similar 
way, definitions can first be definitions-of students' previous activity and later become definitions-for, serving as a tool for further mathematical reasoning. Four layers of activity are inherent in this transition from definitions-of to definitions-for: Situational, Referential, General, and Formal (Gravemeijer, 1999). These four layers, along with distinctions between concept definition and concept image, are central in the DMA framework.

The following forms of definitional activity are present throughout the framework: creating a concept definition, using a concept definition, creating a concept image, and using a concept image. The DMA framework includes Gravemeijer's (1999) four levels of activity, with each level emphasizing two of the four forms of definitional activity (Table 3). To some degree, all four forms of definitional activity are used at each layer, but two of them stand out more than the others at each layer.

\begin{tabular}{|l|l|}
\hline Levels of Activity & Forms of Definitional Activity \\
\hline Situational & Creating a concept definition; Using a concept image \\
\hline Referential & Using a concept definition; Creating a concept image \\
\hline General & Creating a concept definition; Creating a concept image \\
\hline Formal & Using a concept definition; Using a concept image \\
\hline
\end{tabular}

Table 3. Four forms of definitional activity.

Rather than pointing to the discontinuities between a student's concept image and concept definition, the DMA framework describes a trajectory that allows students to make greater and richer connections between their concept image and concept definition.

In situational activity, students use their concept image to create a concept definition. This involves examining prior knowledge as well as taking in new information. These actions involve consideration and refinement of any existing definitions. Thus, to an extent, students are also using concept definitions, and in turn, creating concept images during situational activity. However, at this level, these actions occur much less compared to other levels. 
In referential and general activity, the focus is on creating a concept image. Referential activity involves using a concept definition to create a concept image. This can occur as students interpret a familiar definition in an unfamiliar setting. In general activity, the student is creating (or refining) their concept definition as well as their concept image. Thus, general activity is twofold: students are making generalizations within their concept image based on properties they've noted during referential activity and they are creating their concept definition in light of the new information. As the concept image develops through new situations and experiences, the concept definition should also undergo change. Referential and general activity are important levels of the framework, because at these levels, students are working to create new mathematical realities.

The fourth level of the DMA framework, formal activity, is when the new mathematical reality is largely established and students begin using concept definitions and concept images of the new reality to service other goals. Formal activity involves using concept definitions and concept images that students are familiar with. This is similar to situational activity in which students are also using a familiar concept definition. On the other hand, during referential and general activity, students are in the process of creating new mathematical realities, and do so by using and interpreting a concept definition in a new setting.

Overall, these four types of definitional activity accentuate the interplay between one's concept image and concept definition. These activities evidence that creating a coherent concept image and concept definition is not a trivial matter. Accordingly, a student must be willing to engage in these activities in order to construct well developed understandings. 


\section{Chapter 3 - Theoretical Perspectives}

\subsection{A blend of two perspectives}

A blend of two primary theoretical perspectives were used to analyze and interpret the data for this dissertation study. The first is the theory of concept image and concept definition by Tall and Vinner (1981), which serves as the overarching theoretical perspective for my study, and the second is Zandieh's (2000) derivative framework. A modified version of her framework was also used to analyze data related to tangent lines. The Derivative and Tangent Line Frameworks consist of layers of process-object pairs. Process and object are used in the sense that Sfard (1991) used the terms and these will be formally defined in this chapter. Sfard's notion of pseudostructural conception will also be discussed in this chapter as this was influential to Zandieh's idea of pseudo-objects, which play a key role in the results discussed in this dissertation.

\subsection{Concept image and concept definition}

\subsubsection{Description of theory}

The human brain functions in a complex manner and is not a purely logical entity (Tall \& Vinner, 1981). This type of functioning differs from the logic of mathematics. As Tall and Vinner (1981) explain, "It is not always pure logic which gives us insight, nor is it chance that causes us to make mistakes" (p. 151). Thus, in understanding how students' think and reason mathematically, it is important to make a distinction between formally defined mathematical concepts and the cognitive processes by which they are conceived. For this, I will refer to the theoretical framework of Tall and Vinner (1981) of concept image and concept definition.

Tall and Vinner (1981) use the term concept image to describe "the total cognitive structure that is associated with the concept, which includes all the mental pictures and 
associated properties and processes" (p. 152). An individual's concept image is built up over time. It changes and develops as the individual matures and encounters various experiences. For instance, the concept of tangent line is usually first met in geometry as a tangent to a circle. At this stage, the learner may observe that a tangent line has only one point in common with the shape. This observation becomes part of their concept image for tangent lines and may cause problems later on should they encounter tangent lines to a function graph. All mental attributes associated with a concept belong to the concept image. These associations may be conscious or unconscious and may contain the seeds of future conflict (Tall \& Vinner, 1981).

An individual's concept image need not be globally coherent. Sensory input may prompt certain neuronal pathways while blocking others. In this sense, different stimuli can activate different areas of the concept image. Thus, development of different areas of the concept image can proceed in ways that do not make a coherent whole.

The "portion of the concept image which is activated at a particular time" is called the evoked concept image (Tall \& Vinner, 1981, p. 152). The evoked concept image can be considered a proper subset of the concept image (Meel, 2003). This distinction between the concept image and the evoked concept image enables one to explain how students can respond inconsistently, providing evidence of understanding in one situation and not in another (Meel, 2003). The concept image represents the overall cognitive structure constructed by the learner, but in different contexts, distinct components of the concept image come to the foreground (Meel, 2003). Because one's concept image is not necessarily globally coherent, there may be areas of the concept image that conflict with other areas. These conflicting areas may be evoked at different times due to interaction with different stimuli. It is only when the conflicting areas 
are evoked simultaneously, that the individual would experience a sense of conflict or confusion (Tall \& Vinner, 1981).

It should be evident that one's concept image has the potential to be quite different from a concept's formal definition, if one exists, since a concept image depicts the way the concept is viewed by an individual (Davis \& Vinner, 1986). The concept image includes the various connections of the concept to other associated knowledge structures, prototypical examples, and processes (Meel, 2003). Any concept image has a related concept definition, which is a learner's description of his or her understandings (Meel, 2003). Essentially, the concept definition is regarded as "a form of words used to specify that concept" (Tall \& Vinner, 1981, p. 152). An individual's concept definition may change from time to time. In this sense, a personal concept definition can differ from a formal concept definition, the former being an individualized characterization of the concept and the latter being a definition accepted by the mathematical community at large (Meel, 2003). The concept definition generates its own concept image, the concept definition image, and this is part of the individual's concept image (Tall \& Vinner, 1981).

Tall and Vinner (1981) describe a potential conflict factor as an area of one's concept image or concept definition that is in disagreement with another part of the concept image or concept definition. The individual is most likely unaware of these factors until they are simultaneously evoked and cause a sense of confusion. When this happens, the evoked portions of the concept image are called cognitive conflict factors. Cognitive conflict factors can be evoked subconsciously and demonstrated by a vague sense of unease. It may be a while, if at all, before the learner consciously understands the reason for the conflict. Tall \& Vinner (1981) describe a more serious type of potential conflict factor as one that is at odds not with another 
part of the concept image but with the formal definition of the concept. These factors can seriously obstruct the learning of a formal theory. Students having such a potential conflict factor in their concept image may be secure in their own understandings and regard the formal theory as unnecessary (Tall \& Vinner, 1981). In this case, the learner may never experience cognitive conflict since elements of the formal theory do not belong to their concept image. Cognitive conflict is crucial to the learning process because learners become aware of their misconceptions and can work to reconstruct their understanding (Tall \& Vinner, 1981).

\subsubsection{Concept formation and different thought processes}

Vinner (1991) discusses the difference between the role of definitions in technical and non-technical contexts. For example, one does not consult the definition of "car", "parking lot", or "green" in order to understand the sentence, "among all the cars at the parking lot my green car is the nicest" (p. 67). However, it is necessary to consult definitions when trying to understand the sentence: "among all rectangles with the same perimeter the square is the one which has the maximal area" (pp. 67-68). In technical contexts, people should consult definitions for the technical terms involved. However, Vinner's (1991) work showed that many people ignore definitions in technical settings, just as they are accustomed to doing in non-technical settings. In response to this, Vinner asks the question: "So what do people consult when dealing with technical terms in technical settings?" (p. 68). The remainder of this section summarizes his findings on this issue.

Vinner uses the "scaffolding metaphor" to describe the role of definitions in the formation of the concept image. Definitions help to form the concept image but then become dispensable, similar to the scaffolding being taken away the moment the building construction is complete. When a concept name is seen or heard, it acts as a stimulus and evokes something in 
our memory. Usually what is evoked is not the concept definition but the concept image, or rather an area of the concept image (the evoked portion). It is important to note that the concept image can be translated into verbal forms, but the verbal forms come later and are not what was initially evoked in the memory.

In technical settings, the definition is very valuable (not dispensable!) and has the potential of saving one from traps set up by the concept image. Thus, technical settings impose thought habits on students that are very different from those used in everyday life. Everyday life reasoning flows from one's concept image. These patterns of everyday thinking tend to dominate in technical settings as well, even though this is undesirable from a mathematical standpoint.

For the remainder of this section, assume the existence of two different "cells" in our cognitive structure- one for the definition(s) of the concept and the other for the concept image. It is possible for one or both of the cells to be empty. The concept image cell is considered empty when the learner does not associate anything with the concept name or when the concept definition was learned in a rote and meaningless way. The two cells can develop independently of each other. They also can interact and influence one another.

Sometimes the concept image cell develops first, such as when one is exposed to various examples of a concept prior to knowing its definition. Once the definition is encountered and a concept definition is formed, different types of constructions may take place: i) the concept image may change and be influenced by the concept definition, ii) the concept image may remain the same; the definition cell will contain the memorized definition which will later be forgotten or distorted, iii) the concept image and concept definition may function independently of each other- the learner can provide the definition when needed but in all other instances uses his or her concept image. A similar process might occur when the concept definition develops first. In this 
case, the concept image cell starts out empty and is gradually filled through examples and explanations. The concept image, however, will not necessarily reflect all the aspects of the concept definition. Figure 2 (Vinner, 1991) below represents the interplay between the concept image and concept definition.

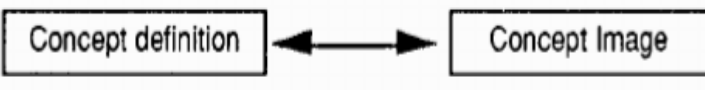

Figure 2. CD and CI inform each other.

Vinner points out that many instructors at the secondary and collegiate level expect a one way process for the concept formation (Figure 3; Vinner, 1991). They expect the concept image to be formed and controlled by the concept definition.

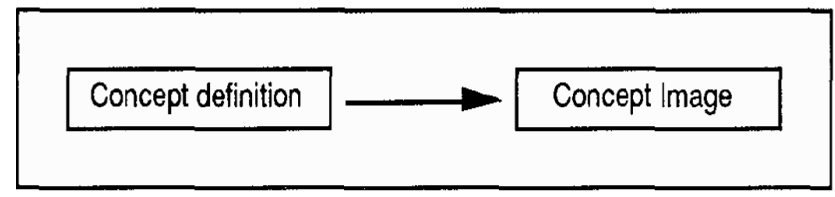

Figure 3. CI formed by CD.

Figures 2 and 3 represent processes of concept formation. In addition, there are also the processes of problem solving and performing tasks (Figures 4, 5, and 6; Vinner, 1991). “Again, it seems to us that many teachers at the secondary and the collegiate level expect that the intellectual processes involved with the performance of a given intellectual task should be schematically expressed by one of the three following figures (the figures represent only the aspect of concept image and concept definition involved in the process)" (p. 71). 


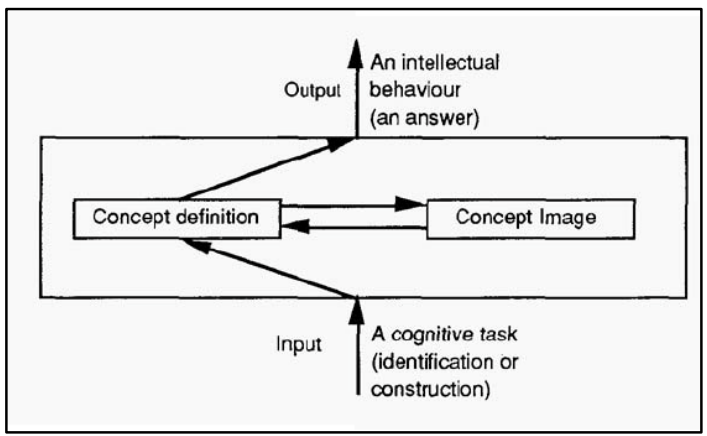

Figure 4. Interplay between definition and image.

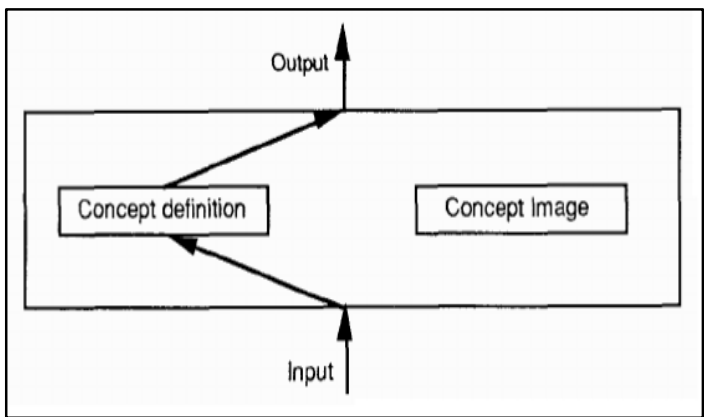

Figure 5. Purely formal deduction.

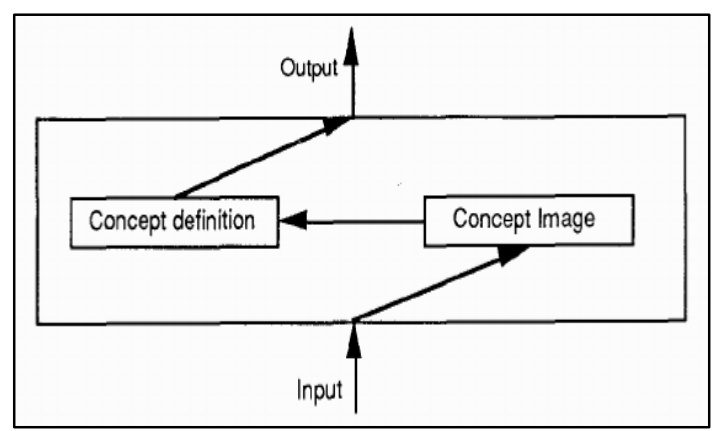

Figure 6. Deduction following intuitive thought.

Each of the above processes consults the concept definition prior to formulating the solution. This is the desirable process and what educators expect and suppose their students are doing. However, Vinner suggests that the reality of it is quite different. He points to the difficulty of training a cognitive system to "act against its nature" by forcing it "to consult definitions either when forming a concept image or when working on a cognitive task" (p. 73). Figure 7 
represents what Vinner calls, "a more appropriate model for the processes that really occur in practice" (p. 73).

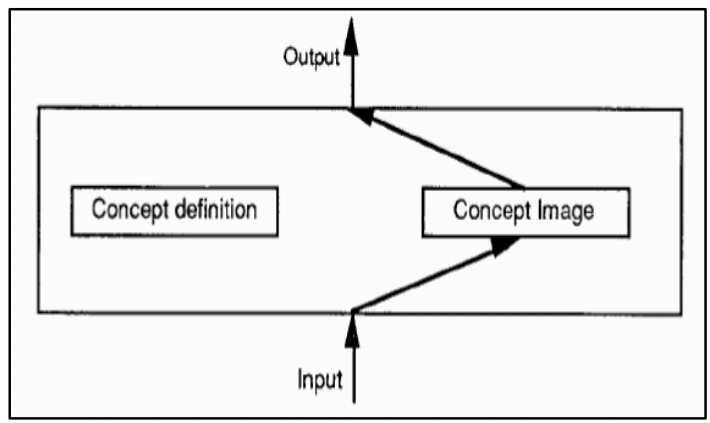

Figure 7. Intuitive Response

In this case, the concept definition cell is not consulted by the learner during the problem solving process. Vinner suggests that the learner is unaware of the need to consult the formal definition because the thinking patterns they use in everyday life do not require them to check formal definitions. Thus, the thinking patterns used in non-technical settings tend to take over in technical settings as well. However, students can still be quite successful (in terms of their grade) in mathematics courses by only referencing their concept image. Vinner points out that only nonroutine problems are capable of unveiling students' incomplete concept images and encouraging them to consult the concept definition. But, students tend to consider these types of problems unfair, and consequently, they are rarely given.

\subsection{Zandieh's Derivative Framework}

My research goals aim to describe students' concept definitions and concept images of the tangent line and consider how these affect students' understanding of the derivative. In order to describe and compare these internal structures, I used a combination of Zandieh's (2000) Derivative Framework and a modified version of her framework for the tangent line. This section outlines these frameworks. 
The frameworks were a means for defining a student's understanding of the tangent line and the derivative in terms of how these understandings align with the formal definitions of the concepts. Additionally, the frameworks served as tools for comparing which aspects of the formal definitions were available to students in different contexts. These understandings represented by the frameworks were analyzed to identify relationships between students' concept definitions of the tangent line and concept images of the derivative. A similar comparative analysis was conducted to analyze students' concept definitions and concept images of the tangent line to see how these internal structures relate.

The next section introduces the three layered Derivative Framework developed by Zandieh (2000), and continues with a description of Sfard's (1991) framework that in part influenced Zandieh's work. The section concludes with details concerning the development of the Tangent Line Framework.

\subsubsection{Layers of process-object pairs}

Zandieh's Derivative Framework has two main components: multiple representations or contexts (the columns) and layers of process-object pairs (the rows). The following quote by Sfard (1991) captures the meaning of object (or structural conception): "Seeing a mathematical entity as an object means being capable of referring to it as if it was a real thing- a static structure, existing somewhere in space and time. It also means being able to recognize the idea at a glance and to manipulate it as a whole, without going into details" (p. 4). Processes, on the other hand, are operations performed on previously established objects. Processes can be conceptually condensed into objects and then acted on by other processes. This forms the chain of process-object pairs described by Zandieh (2000). Essentially, a process-object pair is two ways of looking at the same thing. For instance, the first layer of the Derivative Framework, the 
ratio layer, can be viewed both as a dynamic process- the calculation of the rise over the run of a secant line- and as a static object- the slope of a secant line. Table 4 below shows Zandieh's (2000) diagrammatic representation of her derivative framework, belonging to each cell is a process-object pair. These will be discussed in more detail as this chapter continues.

\begin{tabular}{|c|c|c|c|c|c|}
\hline $\begin{array}{c}\text { Process-object } \\
\text { layer }\end{array}$ & $\begin{array}{c}\text { Graphical } \\
\text { (Slope) }\end{array}$ & $\begin{array}{c}\text { Verbal } \\
\text { (Rate) }\end{array}$ & $\begin{array}{c}\text { Physical } \\
\text { (Velocity) }\end{array}$ & $\begin{array}{c}\text { Symbolic } \\
\text { (Diff. Quotient) }\end{array}$ & Other \\
\hline Ratio & & & & & \\
\hline Limit & & & & & \\
\hline Function & & & & & \\
\hline
\end{tabular}

Table 4. Zandieh's three-layer Derivative Framework.

Her framework describes the derivative concept within four different representations: Graphical (Slope), Verbal (Rate), Paradigmatic Physical (Velocity), and Symbolic (Difference Quotient), and also includes the possibility of other representations. Multiple contexts were incorporated in the design of her framework since research has noted that a person's concept image of the derivative often includes a number of different representations of the concept (e.g. Vinner \& Dreyfus, 1989; Tall \& Vinner, 1981).

This paper focuses on the graphical representation due to the graphical context of this dissertation study. That being said, the remainder of this section considers the graphical column of the above table and outlines the layers and process-object pairs of the framework.

To begin, consider the formal symbolic definition of the derivative:

$$
f^{\prime}(x)=\lim _{h \rightarrow 0} \frac{f(x+h)-f(x)}{h}
$$

"The derivative of $f, f$ ', is a function whose value at any point is defined as the limit of a ratio" (Zandieh, 2000; p. 106). These underlined aspects (Ratio, Limit, and Function) make up the three layers of the derivative concept described by the framework. Each of these layers contains a process-object pair. These pairs form a chain such that the object of one layer is used 
by the process of the next layer. The chain of process-object pairs for the graphical representation are the following:

Layer One (Ratio):

Process: The calculation of the rise over run of a secant line.

Object: The slope of a secant line

Layer Two (Limit):

Process: The limit of a sequence of slope values (these slope values are slopes of secant lines all going through one common point).

Object: The slope of the tangent line at that point.

\section{Layer Three (Function):}

Process: The function process is the notion of running through every point on the original curve and extracting the slope value for the tangent line at that point. (This amounts to running through layers one and two for every point on the function graph.)

Object: The derivative function.

Mathematicians consider the above processes essential for understanding the concept of derivative. These processes are surely not dispensable. Unfortunately, it is noted as "difficult" to elicit student explanations of the ratio and limit processes (Zandieh, 2000; p. 123). Zandieh pointed out that this difficulty may indicate a lack of knowledge or appreciation of these processes on the part of the students. She added that ability to solve problems in calculus may be accomplished without thinking about processes.

The Derivative Framework is meant to describe the mathematical community's notion of understanding the concept of derivative at the first-year calculus level. In turn, it may be used to 
describe aspects of a student's concept image that coincide with the formal definition. More specifically, the framework may be used to note whether a student has developed the processobject aspects of the concept of derivative.

If a student has not developed an understanding of the processes which formally define the concept, their understanding is considered pseudostructural. In these cases, students think about the derivative in terms of pseudo-objects. A pseudo-object is defined as "an intuitive understanding that does not involve an understanding of the process underlying the object" (Zandieh, 2000; p. 124). Zandieh points out that a person may use a pseudo-object because he or she does not know the underlying process or may do so because there is no need to refer to the underlying process in the context in which the person is working. The latter is a natural, and often necessary way, to work with mathematical objects, whereas the former is problematic and often representative of an underdeveloped understanding. It is suitable to point out that the participants of my study were encouraged to fully describe their understandings. Therefore, the context in which they were working was not one in which there was "no need" for them to refer to the underlying processes. By prioritizing the processes underlying the derivative concept, Zandieh's framework assisted in considering pseudostructural aspects of students' concept images.

As illustrated in the above review, Zandieh's framework puts forth the notion of describing student understanding of the concept of derivative in terms of three process-object pairs. In this way, the Derivative Framework, and the framework for tangent lines (outlined later in this chapter), were useful guides for identifying elements of students' concept images and labeling these elements as processes or potential pseudo-objects. 
In addition to serving as a tool for describing student understanding, Zandieh (2000) points out that the process-object framework may be used to note whether a textbook or a set of curriculum materials provides students opportunities to learn the underlying processes in multiple contexts. Additionally, it may be used to analyze classroom discourse for which aspects of the concept of derivative are involved in teacher questions and descriptions. In these ways, the framework is broader than a tool for characterizing students' concept images.

Mathematically, it is clear that the derivative concept involves a ratio, a limit, and a function. Zandieh's idea of these being layers developed from her thinking about Sfard's (1991) framework of processes acting on previously established objects. The next section briefly describes Sfard's (1991) framework. The review does not discuss the details of Sfard's theory of concept development: interiorization, condensation, and reification, but focuses only on her notions of processes, objects, and pseudostructural conceptions, which are key elements of Zandieh's framework.

\subsubsection{Sfard's theory of processes and objects}

If a student has not developed a structural conception of one of the layers of the framework, the question arises as to how they will consider the next process in the framework without an object on which to operate. One solution is that they will proceed by means of a pseudostructural conception. As previously mentioned, Zandieh termed “objects” associated with this type of conception as pseudo-objects. Pseudo-objects may be thought of as objects with no internal structure. However, it should be pointed out that pseudo-objects are not meant to always carry a negative connotation. For example, it is often convenient to describe a process by having it operate on a pseudo-object. This way, one does not have to go into detail concerning all the internal processes of the object. However, this way of thinking should be only for 
convenience and not represent the extent of one's understanding. Sfard (1991) refers to both operational and pseudostructural conceptions as "deviations from the official structural approach" (p. 77). She explains that while operational conceptions are "a healthy, natural stage in concept development," pseudostructural conceptions should be considered "unsatisfactory and potentially harmful" (p. 77).

The terms operational and structural are not to be thought of as separate ideas, as if mathematical notions are either operational or structural. Rather, they are to be viewed as "inseparable, though dramatically different, facets of the same thing" (1991, p. 9). In this sense, the process-object pairs of the layers of the frameworks (derivative and tangent line) represent different ways of looking at the same thing. Each of the layers- Ratio, Limit, and Function- are considered to be both dynamic processes and static objects.

An important aspect of Sfard's theory as it relates to Zandieh's framework, is Sfard's suggested model of concept formation implies that "certain mathematical notions should be regarded as fully developed only if they can be conceived both operationally and structurally" (1991, p. 23). Thus, a student's understanding of the derivative concept (or the tangent line, described next) is not regarded as fully developed unless they are able to conceive of each layer of the framework both operationally and structurally.

\subsubsection{Tangent Line Framework}

The Tangent Line Framework of this paper is a subset of Zandieh's (2000) Derivative Framework. This is represented by the highlighted region of Table 5 on the next page. 


\begin{tabular}{|c|c|c|c|c|c|}
\hline $\begin{array}{c}\text { Process-object } \\
\text { layer }\end{array}$ & $\begin{array}{c}\text { Graphical } \\
\text { (Slope) }\end{array}$ & $\begin{array}{c}\text { Verbal } \\
\text { (Rate) }\end{array}$ & $\begin{array}{c}\text { Physical } \\
\text { (Velocity) }\end{array}$ & $\begin{array}{c}\text { Symbolic } \\
\text { (Diff. Quotient) }\end{array}$ & Other \\
\hline Ratio & & & & & \\
\hline Limit & & & & & \\
\hline Function & & & & & \\
\hline
\end{tabular}

Table 5. Tangent Line Framework, subset of Zandieh's Derivative Framework.

Whereas the Derivative Framework is made up of three layers- Ratio, Limit, and Function, the Tangent Line Framework is made up of two- Ratio and Limit. It was developed by integrating the formal definition of the tangent line at the first-year calculus level into the layered structure of Zandieh's Derivative Framework. The definition of the tangent line fits naturally into the layered structure given the mathematical relationship of these two concepts. The processobject pairs parallel those in the derivative framework. The similar structures of the frameworks provided a meaningful way to compare students' understandings of the tangent line to that of the derivative. The development of the Tangent Line Framework is demonstrated next.

To begin, consider the formal definition of the tangent line:

Definition: The tangent line to the curve $y=f(x)$ at the point $P(a, f(a))$ is the line through $P$ with slope

$$
m=\lim _{x \rightarrow a} \frac{f(x)-f(a)}{x-a}
$$

provided that this limit exists. (Stewart, 2015, p. 141)

The first layer is the ratio process. To start, the process uses two objects- two differences, $f(x)-f(a)$ and $x-a$, and acts by division. The division action produces a slope. This slope is the object of the first layer, and it graphically represents the slope of a secant line through the curve. This object of the first layer is used by the limiting process in the next layer. The limiting process "passes through" infinitely many of the slopes approaching a particular 
value. This value, $m$, if it exists, is the object of the second layer and is graphically understood to be the slope of the tangent line to the curve at the point $(a, f(a))$. Thus, the formal definition of the tangent line, like the derivative concept, can be thought of as a chain of process-object pairs. Table 6 outlines the details of the layers of the Tangent Line Framework.

\begin{tabular}{|l|l|}
\hline \multicolumn{1}{|l|}{ Tangent Line Framework, Graphical Representation } \\
\hline $\begin{array}{l}\text { If a curve } C \text { has equation } y=f(x) \text { and we want to find the tangent line to } C \text { at the point } P(a, f(a)) \ldots \\
\text { Layer One } \\
\text { Ratio) }\end{array}$ & $\begin{array}{l}\text { Process: consider a point } Q(x, f(x)) \text {, where } x \neq a \text {. The slope of the secant line } P Q \\
\text { will be }\end{array}$ \\
$\qquad m_{P Q}=\frac{f(x)-f(a)}{x-a}$ \\
$\begin{array}{l}\text { Layer Two } \\
\text { (Limit) }\end{array}$ & $\begin{array}{l}\text { Object: The secant line through points } P \text { and } Q \text { with slope } m_{\mathrm{PQ}} . \\
\text { the tangent } T \text { to be the line through } P \text { with slope }\end{array}$ \\
& $\begin{array}{l}\text { Oby letting } x \text { approach } a \text {. We define } \\
\text { Object: The tangent line with slope } \mathbf{m} \text { at the point } P(a, f(a)) .\end{array}$ \\
\hline
\end{tabular}

Table 6. Layers of process-object pairs for the Tangent Line Framework.

The phrases shown in bold font are considered the objects of the respective layers.

Whereas Zandieh's framework considered slope to be the object of layers one and two, the Tangent Line Framework incorporates the secant line, the tangent line, and one point as objects of the layers. This feature of the framework differs from what Zandieh described in her Derivative Framework. Since the definition of the tangent line amounts to saying, "the tangent line is the limiting position of secant lines (Stewart, 2015, p. 141)" it makes sense to consider the tangent line the resulting object of the limiting process of secant lines, as well as one point the resulting object of the limiting process of one point approaching another point.

Similar to Zandieh's Derivative Framework, the Tangent Line Framework also functions as a chain- the object of one layer used by the process of the next layer. Figure 8 on the next page illustrates this feature of the framework. 


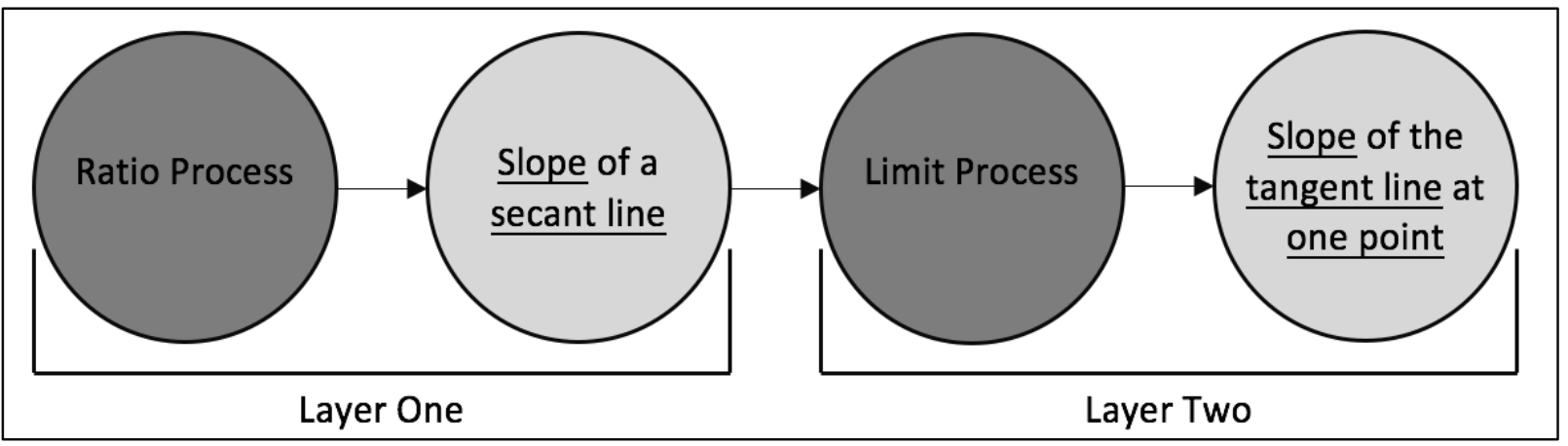

Figure 8. Tangent Line Framework. Chain of process-object pairs.

Zandieh outlines a procedure of open and shaded circles for coding the understanding demonstrated by a student into the matrix for the framework (Table 4). Open circles are used to illustrate a student's mention of the object for a layer (represented by the lightly shaded circles in the above figure), and shaded circles are used to denote a student's explanation of the process for a layer (represented by the darker shaded circles in the above figure). Thus, if a student mentions only the object of a layer without describing the underlying process, then the open circle of their coded matrix represents a potential pseudo-object. This is indicative of a pseudostructural understanding for that aspect of the framework. This feature of the analysis is captured in the above diagram.

Since the Derivative and Tangent Line Frameworks describe the mathematical community's notions of understanding the concepts, these were useful for describing students' access to aspects of the formal definitions. Also, since the Tangent Line Framework is a subset of the Derivative Framework, these were convenient for compare students' understandings across concepts. Additionally, the Tangent Line Framework was useful for comparing students' concept definitions and images of the tangent line.

\subsection{Summary}

My research project incorporates a blend of theoretical perspectives. Tall and Vinner's (1981) theory of concept image and concept definition serve as the overarching explanation for 
the understandings demonstrated by the students. These understandings were further characterized using Zandieh's (2000) Derivative Framework and my modified version of it for tangent lines. 


\section{Chapter 4-Methods}

\subsection{Data collection}

A series of semi-structured task-based clinical interviews (Goldin, 2000) were conducted over the course of the spring 2015 semester with twelve West Virginia University first-semester calculus students. This section looks at details concerning the design and implementation of the study as well as demographics of the involved participants.

\subsubsection{Pilot studies}

As referenced in the introductory chapter, two pilot studies were conducted during the spring 2013 and fall 2014 semesters with significantly different results in terms of students' abilities to correctly construct tangent lines. The first of these studies examined students' understanding of Newton's Method, which involves applications of tangent lines. These students struggled to comprehend Newton's Method because of their difficulties with the tangent line. The 2014 study was a follow-up study and examined how first-semester calculus students think about the tangent line. The students of this study did not experience the same difficulties.

This gap in understanding led us to consider the dynamics of each study and identify factors that may have played a role in the contrasting results. We hypothesized that the differences were due to the timing of the studies. The first study took place closer to the middle of the semester, when the students were learning applications of tangent lines, whereas the second study took place closer to the beginning of the semester, immediately following instruction on tangent lines. We hypothesized that the students in the mid-semester study struggled with tangent lines because they were working to construct understandings of applications of tangent lines, which presented extra layers of information. Thus, their preexisting knowledge for constructing tangent lines became somewhat clouded by these efforts. In 
other words, their efforts to understand and organize numerous layers of information had a negative effect on their previous understandings. In contrast, the students in the beginningsemester study who were at the initial phase of the learning process and were unencumbered by the extraneous information.

\subsubsection{Overview of dissertation study}

In response to these results, this dissertation study was designed to examine first-semester calculus students' conceptions of the tangent line over the course of a semester. Where the pilot studies provided a single snapshot of a student's understanding at a given point in time, this study was designed to document several snapshots of a student's understanding so that comparisons could be made across time.

To collect relevant data, the design of this study focused on two primary factors affecting students' understandings of the tangent line: time and applications. To address these two factors, a series of three interviews was conducted over the course of a semester and the types of tasks evolved for each interview, beginning with tasks related only to tangent lines and progressing to more complicated tasks involving applications of tangent lines. In this way, Interview 1 modeled the 2014 pilot study since it was at the beginning of the semester and focused on tangent lines, and Interviews 2 and 3 were intended to model the 2013 pilot study since they occurred later in the semester and incorporated tasks involving applications of tangent lines.

\subsubsection{Sample selection and participant demographics}

To recruit participants for the study, I attended an evening class mandatory to all of the non-engineering sections of first-semester calculus and talked to the students about the research project, asking for volunteers. I explained that the study consisted of a series of interviews and emphasized that students should agree to participate only if committed to completing the entire 
series. Students were informed that if they missed one of the interviews, they would not be permitted to take part in any of the remaining interviews. Since students were required to participate in not just one but several interviews, a monetary incentive was offered to encourage student participation: $\$ 10$ for each interview and a $\$ 20$ bonus for completing the entire series. Choosing to participate in the study had no impact on students' grades in their calculus course.

Those interested were given a date, time, and location to complete a background questionnaire. The questionnaire consisted of fourteen questions, half of which concerned tangent lines and half of which were demographic or background questions, such as their major, previous math courses taken, or their confidence level as a mathematics student. Thirty students participated in the questionnaire, and soon after, were contacted via email to sign up for the first interview of the series by way of an online calendar. Of those thirty students, twenty attended the first interview. Those twenty students were again contacted via email and asked to use the same process to sign up for the next interview. Of those twenty, twelve participated in the second interview. These final twelve students also completed the third interview of the series.

The twelve subjects of this dissertation study consisted of six males and six females. To protect their identities, I will use the names Aaron, Colton, Corey, Jacob, Kenneth, and Kevin for the six males and the names Alice, Amanda, Anita, Carly, Jamie, and Mindy for the six females. All twelve students' data contributed to the results discussed in this dissertation, but four of these students were analyzed in greater detail. These are referred to as the core students of this study, namely, Aaron, Alice, Colton, and Jamie (The process for selecting these four out of the twelve is reviewed in the Data Analysis section of this chapter.) Table 7 summarizes results from the questionnaire related to the twelve participants' mathematics background, year, and major. 


\begin{tabular}{|c|c|c|c|c|c|}
\hline Student & $\begin{array}{l}\text { Confidence } \\
\text { level }\end{array}$ & Year & Major & $\begin{array}{l}\text { Time since } \\
\text { passed math } \\
\text { course with C } \\
\text { or higher }\end{array}$ & $\begin{array}{l}\text { Previous calculus } \\
\text { experience }\end{array}$ \\
\hline Aaron & 3 & Freshman & Biology & $1-2$ Years & $\begin{array}{l}\text {-Calculus } 1 \text { (WVU) } \\
\text {-Calculus course in high } \\
\text { school (not AP) }\end{array}$ \\
\hline Alice & 3 & Freshman & Forensics & $\begin{array}{l}\text { Less than one } \\
\text { year ago }\end{array}$ & First time taking calculus \\
\hline Colton & 2 & Sophomore & $\begin{array}{l}\text { Mechanical } \\
\text { Engineering }\end{array}$ & $\begin{array}{l}\text { Less than one } \\
\text { year ago }\end{array}$ & $\begin{array}{l}\text {-Calculus 1a with Pre- } \\
\text { calculus (WVU) } \\
\text {-Calculus } 1 \text { (WVU) }\end{array}$ \\
\hline Jamie & 2 & Freshman & Psychology & $\begin{array}{l}\text { Less than one } \\
\text { year ago }\end{array}$ & First time taking calculus \\
\hline Amanda & 3 & Sophomore & Psychology & $\begin{array}{l}\text { Less than one } \\
\text { year ago }\end{array}$ & AP Calculus in high school \\
\hline Anita & 4 & Freshman & $\begin{array}{l}\text { Animal and } \\
\text { Nutritional } \\
\text { Science } \\
\end{array}$ & $\begin{array}{l}\text { Less than one } \\
\text { year ago }\end{array}$ & First time taking calculus \\
\hline Carly & 2 & Senior & Biology & $1-2$ years ago & AP Calculus in high school \\
\hline Corey & 4 & $\begin{array}{l}\text { Graduate } \\
\text { Student }\end{array}$ & $\begin{array}{l}\text { Mathematics } \\
\text { Education }\end{array}$ & $2-5$ years ago & Applied Calculus (WVU) \\
\hline Jacob & 3 & Senior & Accounting & $2-5$ years ago & Applied Calculus (WVU) \\
\hline Kenneth & 4 & Freshman & $\begin{array}{l}\text { Mathematics } \\
\text { Education }\end{array}$ & $\begin{array}{l}\text { More than } 5 \\
\text { years ago }\end{array}$ & Calculus 1 (WVU) \\
\hline Kevin & 1 & Senior & Psychology & $1-2$ years ago & $\begin{array}{l}\text {-Calculus 1a with Pre- } \\
\text { calculus (WVU) } \\
\text {-Calculus 1 (WVU) }\end{array}$ \\
\hline Mindy & 2 & Freshman & Biology & $1-2$ years ago & First time taking calculus \\
\hline
\end{tabular}

Table 7. Participant information.

Four of the twelve participants were taking calculus for the first time, while the remaining eight had previously taken calculus in high school or were repeating the course for reasons not addressed by the questionnaire. The average confidence level in mathematics of the twelve students was a 2.75 on a 4 point scale with 4 being very confident.

Each of the twelve subjects were enrolled in non-engineering first-semester calculus at West Virginia University during the spring 2015 semester, the time of the study. The students were not all from the same calculus class but were divided among five calculus instructors. However, the calculus courses in which the students were enrolled were coordinated in that the same material was covered among all sections and was covered around the same time frame. 


\subsubsection{Interview process}

The following description outlines how the questionnaire and series of interviews were spaced over the course of the semester (spring 2015):

1. Questionnaire: February $3^{\text {rd }}, 2015$. Retrieve background information, prior to classroom instruction on tangent lines.

2. Interview 1 : February $9^{\text {th }}-13^{\text {th }}, 2015$. Immediately following in-class instruction on tangent lines.

3. Interview 2: March $16^{\text {th }}-19^{\text {th }}, 2015$. Following in-class instruction on applications of tangent lines.

4. Interview 3: April $27^{\text {th }}-30^{\text {th }}, 2015$. End of semester, prior to final examination.

All interviews were video recorded and lasted approximately twenty-five minutes. Those present during the interviews were the student, the interviewer, and a video camera operator. For some of the interviews, the interviewer managed the video camera, and so a third person was not present. Two graduate students were recruited to assist with data collection. Typically, I was the interviewer, and one of the two graduate students operated the video camera, but the graduate students also conducted interviews. In this way, switching interviewers may have impacted the performance of the students. They may have felt more comfortable with me since I was leading the project and more involved in the interview process. Or, quite possibly, they may have felt more comfortable with the two graduate students assisting with my research project since their role was perhaps less intimidating. Either way, the alternating approach to conducting the interviews may have affected the responses provided by the participants. The interviews took place in either a small conference room or an average sized classroom on campus. In either case, the room was free from distractions, and the students did not have access to textbooks or other 
materials to assist in answering interview questions. They were provided handouts relevant to the interview protocol, scrap paper, pencils, pens, and rulers (to assist with constructing tangent lines).

It was important to conduct the interviews in such a way that the students were comfortable to provide honest answers. In an attempt to create such an environment, students were often assured that the interviews were not a test or an assessment; students were encouraged to talk freely about what they were thinking. When problem solving, students were asked to think out loud and vocalize their thought processes. It was important to keep the interviews informal, while maintaining an environment in which the participants felt motivated. To do so, the interviews focused on a limited number of tasks. This way, students should not have felt rushed or pressured to work quickly, but could take their time to search their concept images for necessary information.

Table 8 reviews the four primary tasks for each interview, and these are also available for review in Appendices B, C and D.

\begin{tabular}{|l|c|c|c|}
\hline Tasks & Interview 1 & Interview 2 & Interview 3 \\
\hline Task 1: Definition of the tangent line & $\mathrm{X}$ & $\mathrm{X}$ & $\mathrm{X}$ \\
\hline Task 2: Construction of tangent lines (six graphs) & $\mathrm{X}$ & $\mathrm{X}$ & $\mathrm{X}$ \\
\hline $\begin{array}{l}\text { Task 3: Sketch the derivative for a given function } \\
\text { graph (algebraic representation not provided) }\end{array}$ & & & $\mathrm{X}$ \\
\hline $\begin{array}{l}\text { Task 4: Graphically interpret the formal symbolic } \\
\text { definition of the derivative }\end{array}$ & & & $\mathrm{X}$ \\
\hline
\end{tabular}

Table 8. Summary of interview tasks.

Interview 1 included a few additional construction tasks in addition to the six graphs, and Interview 2 included an opening question about Newton's Method. These tasks were considered during beginning phases of analysis, but the majority of data for this dissertation comes from the four primary tasks in the table. 
Examining the columns of the table, notice that each interview was essentially a repeat of the previous one, with one additional task. The first interview consisted of tasks involving only tangent lines, whereas the second and third interviews included additional tasks related to applications of tangent lines. By changing the tasks overtime, I were able to analyze changes in students' concept images of tangency. In particular, these methods were implemented in order to examine the hypothesized factors of time and application from the differing results of the pilot studies. Would these students mimic those results and demonstrate weakening understandings as they encountered the tangent line in mathematically richer contexts?

Examining the rows of Table 8, notice that Tasks 1 and 2 were repeated for each interview- defining and constructing tangent lines. By students providing their concept definition for each interview, I was able to gauge how these increased or decreased in sophistication over the semester. Likewise, the six graphs task provided data for analyzing students' abilities to construct tangent lines overtime. By the second and third interviews, the students would see the six graphs and respond, "Not these again!" Whereas Task 1 represented students' concept definitions of the tangent line, Task 2 represented their concept images of the tangent line. These two sets of data were comparatively analyzed to examine how students' concept definitions and images relate to each other.

The four interview tasks were connected in that the first two showed us how students think about the tangent line and the last two showed us how students think about the derivative. These understandings were analyzed using the Derivative and Tangent Line Frameworks (Outlined in Chapter 3). In seeking to describe a student's concept image of the tangent line and the derivative in terms of alignment with these frameworks, my analysis considered what aspects of the frameworks were available to each student. What processes or objects did the student 
mention? Did they connect the limit process of the tangent line to the limit process of the derivative? Using the frameworks, students' understandings of the tangent line and the derivative were compared, and interpretations were made concerning how the structure of students' concept definitions of the tangent line affect their understanding of the derivative concept.

The research methods described in this section were aimed at collecting the following information:

- The students' concept definitions of the tangent line- regarded as "a form of words used to specify that concept" (Tall \& Vinner, 1981, p. 152). (Task 1)

- Descriptions of each student's concept image of the tangent line in terms of its overlap or lack of overlap with the Tangent Line Framework. (Task 2)

- Descriptions of each student's concept image of the derivative in terms of its overlap or lack of overlap with the Derivative Framework (Zandieh, 2000). (Tasks 3 and 4)

- An examination of the structure of each students' concept image of the tangent line and the derivative and how these concept images are related. (Comparative analysis- Tasks 1 and 2 to Tasks 3 and 4)

- An examination of differences in the understanding demonstrated by each student based on the mathematical situation.

- An examination of any instances of cognitive conflict and documentation of the evoked elements of the concept image that contributed to this conflict. 


\subsection{Data analysis}

The last section described the design of my dissertation study and the process of data collection. This section looks at the details concerning the analysis of the collected data. It begins with a review of the grounded theory approach to data analysis developed by Strauss and Corbin (1990) and continues with a description of the three-phase analytic process for my dissertation study.

\subsubsection{Grounded theory}

To analyze the data, I used the grounded theory approach to data analysis described by Strauss and Corbin (1990). This approach is valuable in its ability to both generate theory and ground that theory in data. Their theory of qualitative analysis is a process of interpretation "carried out for the purpose of discovering concepts and relationships in raw data and then organizing these into a theoretical explanatory scheme" (p. 11). The focus is on allowing the theory to emerge from the data. This approach to analysis involves Open, Axial, and Selective Coding as well as identifying properties and dimensions of categories. These types of analysis are not necessarily sequential steps but proceed quite naturally together. Each type of coding utilizes the analytic tools of Asking Questions and Making Theoretical Comparisons, which Strauss and Corbin (1990) describe as being essential for the development of theory.

For organizational purposes, I have divided this section into three phases of analysis, each phase building on the previous. In discussing each phase, I hope to accurately convey the techniques and procedures of analysis that I used while also elaborating on the grounded theory of Strauss and Corbin (1990).

Each phase of data analysis consisted of cycles of moving from the raw data, to the level of the abstract, and back to the raw data for validation and further analysis. Strauss and Corbin 
(1990) note that this interplay between making inductions (deriving concepts, their properties, and dimensions from the data) and deductions (hypothesizing about the relationships between concepts) is at the heart of theorizing. Throughout each phase of analysis, I strived to follow the advice of Strauss and Corbin (1990) of thinking creatively while maintaining a stance of objectivity and sensitivity towards the data.

\subsubsection{Phase 1- open and axial coding}

At the conclusion of data collection, I had a total of thirty-six interviews for analysistwelve students, three interviews each. To familiarize myself with the data, I viewed the series of interviews for each participant and assigned each interview a rating based on the student's performance (excellent, good, ok, or poor). Strauss and Corbin (1990) refer to this type of coding as "perusing the entire document" (p. 120). The following questions guided my analysis in assigning ratings: What are their concept definitions of the tangent line? Are they successful at completing the interview tasks? What level of explanation are they able to provide to justify their work?

Because the pilot studies yielded dramatically different results, similar expectations were in place for the ratings across the series. However, the participants' ratings remained fairly consistent. In some cases, the rating increased or decreased across interviews by at most one level, for example poor to ok or ok to good; the changes were never major, such as excellent to poor, which was the expectation in place from the pilot studies. Even at such a beginning stage of analysis, it was increasingly evident that our hypothesis of change from the pilot studies was not holding up under the new data. While there was some evidence of change, this change was insignificant compared to the results of the two pilot studies in that the students' performances in the pilot studies were dramatically different; students in one study could barely construct correct 
tangent lines while students in the other study almost always constructed correct tangent lines. The students of my dissertation study remained mostly consistent in their abilities to construct tangent line over the interview series.

The research questions I had set out to explore were somewhat trivially answered in that the change we were expecting was not happening. This led to the redevelopment of my originally proposed research questions. The methods of my study remained the same. What the data was conveying changed the questions I was asking. The development of this redirection is highlighted in this section.

Setting aside preconceived notions, I proceeded with data analysis through multiple viewings of the interviews. During this phase of open coding (identifying concepts and their properties and dimensions in the data), my goal was to let the data speak as I noted emerging concepts and possible categories. Many of the codes that emerged during this time were descriptors of how students' defined the tangent line. These were grouped together under the category Concept Definition of Tangent Line, and moving forward with analysis, I sought to discover properties and dimensions of this category in the data.

During this process, I made use of the analytic tool of diagramming discussed by Strauss and Corbin (1990). Diagramming was helpful at this stage, because it enabled me to gain some distance from the data and work at a more conceptual level. For example, one diagram consisted of a chart that served as a summary of the students' concept definitions of tangency across each interview. The rows of the chart displayed the students' names and the columns were labeled Interview 1, Interview 2, and Interview3. The chart was representative of all thirty-six interviews and documented each students' language for defining a tangent line. The chart also included additional notes that described aspects of students' concept images of tangency, such as their 
ideas on the relationship between tangent lines and the derivative, how they described places where a tangent line did not exist, how they determined the slope of the tangent line, the language they used to justify their work throughout the interviews, or anything they said that was particularly interesting. Constructing the chart allowed me to process the data at a more conceptual level. A striking theme began to emerge from the data- a connection between students' concept definition of the tangent line and their concept image of the derivative. Through the analytic process of diagramming, Concept Image of Derivative emerged as a second category.

Recognizing a connection in the data led to the process of validating my interpretations. To do so, I followed Strauss and Corbin's (1990) guidelines of making comparisons and asking questions. During this phase of axial coding (relating categories to subcategories and identifying relationships across categories), I compared students' definition of the tangent line (Interview 3, Tasks 1) to their understandings of the derivative (Interview 3, Tasks 3 and 4) and considered: What aspects of the Tangent Line Framework are represented in the data? What aspects of the Derivative Framework are represented in the data? How do these relate to each other? The following subcategories and properties related to the frameworks were used to develop the categories:

Subcategory1: Process

Properties: Ratio, Limit, Function

\section{Subcategory2: Object}

Properties: Slope, One Point, Derivative

Other concepts emerged during this process that described how students' thought about tangent lines, for example, Slope Zero, Curve, Concavity Change, Guessing, and Class. These 
codes were representative of the language used by the students when constructing tangent lines. Codes that incorporate the subjects' language are called in vivo codes.

In summary, Phase 1 of data analysis included both open and axial coding and made use of the analytic tools of thinking comparatively, asking questions, and diagramming. Two categories were identified: Concept Definition of Tangent Line and Concept Image of Derivative, and were analyzed in terms of subcategories and properties. Because change in students' understanding of the tangent line across the series of interviews was not a striking theme in the data, my originally proposed research questions were modified. As a review, the research questions noted in my dissertation proposal were the following:

1. What transformations do students' concept images of the tangent lines undergo over the course of fist-semester calculus?

2. What role does cognitive conflict play in the development of students' concept image of tangent lines?

The data of my dissertation study told a different story from what I encountered in the pilot studies. The reasons for this are not clear, but rather than pursuing explanations for these differences, my attention was refocused based on what I was seeing in the data- a very interesting relationship between how a student describes the tangent line and how they perform on tasks related to the derivative. In order to explore this emerging theme, I revised my research questions. To review, the research questions of this dissertation are the following:

1. What are first-semester calculus students' concept definitions and concept images of the tangent line? How do these relate to one another and compare to the formal definition of the tangent line at the first-semester calculus level? 
2. How do first semester calculus students connect the concepts of the tangent line and the derivative in a graphical representation?

The remainder of this chapter considers that data analysis for answering these two questions.

Restructuring the research questions was a necessary step that allowed me to move forward with analysis in a way that felt both meaningful and consistent with what was indicated in the data. Strauss and Corbin (1990) sum it up quite perfectly:

"And no matter how well thought out we think our project is at the beginning, there are always those unanticipated twists and turns along the way that lead us to rethink our positions and question our methods and to let us know that we are not as smart as we think we are.” (p. 55)

\subsubsection{Phase 2- selective coding}

Twelve students completed the series of three interviews. Phase 1 of analysis consisted of rating each of the thirty-six interviews (poor, ok, good, or excellent), creating a diagram that summarized students' concept definitions of the tangent line and documented their language for describing the derivative concept, and multiple viewings of each of the interviews. Through open and axial coding, the relationship between students' concept definition of the tangent line and concept image of the derivative became a pursuable theme in the data.

Moving into the second phase of analysis, I focused my attention on an in-depth analysis of a subset of the twelve subjects. Four students were selected by considering average interview ratings for the series, previous calculus experience, and the level of detail provided in the students' explanations during the interviews. The students of each average rating class are shown in the next table, with the four students shown in bold (Table 9). These are referred to as the core students- Aaron, Alice, Colton, and Jamie. 


\begin{tabular}{|l|l|}
\hline Ratings & Students \\
\hline Excellent & Aaron \\
\hline Good & Amanda, Carly, Corey, Colton \\
\hline Ok & Alice, Jacob, Mindy, \\
\hline Poor & Anita, Jamie, Kenneth, Kevin \\
\hline
\end{tabular}

Table 9. Average interview ratings and four core students.

Both Aaron and Colton had previous calculus experience, while Alice and Jamie were taking calculus for the first time. These four students also stood out in that the explanations they provided were quite lengthy compared to the other students in their rating class. This was helpful for analysis since more data would be available.

Once the subset of the subjects was selected, I continued axial and open coding for this subset of participants. To begin, I transcribed the series of three interviews for each of the four students, while keeping a journal that documented any thoughts or insight.

Strauss and Corbin (1990) describe microscopic analysis (microanalysis) as a technique for opening up the text, and point out that this type of analysis compels the analyst to "listen closely to what the interviewees are saying and how they are saying it" (p. 65). After transcribing the series of interviews for the four core participants, I began a line by line analysis of Jamie and Aaron's series. Aaron's interview series had an overall rating of excellent and Jamie's an overall rating of poor. I paid particular attention to the language used by the students, asking: What language is Aaron using in his descriptions that Jamie is not? Why is Aaron more successful at the interview tasks than Jamie? What distinguishes Aaron's interviews from Jamie's?

I constructed a language chart for Jamie and Aaron that consisted of quotes from each of their transcripts in describing the tangent line and derivative. The diagram assisted with developing categories in terms of dimensions (range along which general properties of a category vary). For instance, the property slope was characterized in terms of type: slope of graph, slope 
of zero, slope of tangent line. The diagram was also useful for identifying emergent themes and generating new codes. For example, I noticed that Aaron often referenced his concept definition of the tangent line when solving the interview tasks, whereas Jamie did not. Referencing became an emergent theme in the data. The in-depth analysis of Jamie and Aaron helped generate codes that could be explored in the remaining students' data.

Examining students' interview transcripts with the developed codes allowed me to group and refine the codes. During this phase of selective coding (integrating and refining the theory), I stayed open to identifying new codes and organizing these into the coding scheme. For instance, cognitive conflict was an emergent theme from Colton's data and was incorporated into the coding system.

To compare students' understandings per task and per interview, the Derivative and Tangent Line Frameworks were used to organize the data (Table 10). Using the frameworks, a comparative analysis was completed across interviews, concepts, and tasks.

\begin{tabular}{|c|c|c|c|c|}
\hline \multicolumn{2}{|l|}{$\begin{array}{l}\text { Charts for Tangent Line and Derivative } \\
\text { Frameworks }\end{array}$} & $\begin{array}{c}\text { Layer One } \\
\text { (Ratio) }\end{array}$ & $\begin{array}{c}\text { Layer Two } \\
\text { (Limit) }\end{array}$ & $\begin{array}{c}\text { Layer Three } \\
\text { (Function) }\end{array}$ \\
\hline \multirow{2}{*}{ STUDENT } & Tangent Line & & & N/A \\
\cline { 2 - 5 } & Derivative & & & \\
\hline
\end{tabular}

Table 10. Tangent Line and Derivative Frameworks.

Recall, the frameworks represent the mathematical community's notions of understanding the concepts of the derivative and the tangent line. These define the concepts in terms of layers of process-object pairs. If a student referenced the object of a layer, an open circle was placed in the corresponding cell, and if a student demonstrated an understanding of the process of a layer, a shaded circle was placed in the corresponding cell. The Tangent Line Framework is a subset of the Derivative Framework and was designed to analyze students' understandings of the tangent line and compare these to how they think about the derivative. However, a key distinction between the frameworks should be addressed- the Derivative 
Framework has multiple representations (slope, rate, velocity, and symbolic), whereas the Tangent Line Framework has just one, graphical. The expansion of the framework is a proposed endeavor for future research projects, but for the purposes of this study, I have focused on the graphical context of the tangent line for primarily two reasons: 1) Consistency with the type of tasks proposed in the interviews (graphical) and 2) Students explanations were overwhelming graphical (as evidenced by Tables 37 and 38 at the conclusion of the Data Chapter). Some students, particularly Colton, demonstrated understandings of the tangent line outside of its graphical meaning, such as instantaneous rate of change or velocity. These understandings were not captured by the framework but were documented as belonging to his concept image of the tangent line and were incorporated in the analysis of his data.

In regard to the hypothesis of change from the pilot studies, the completed charts verified that students' concept images of the tangent line did not undergo significant change over the series of interviews.

In summary, Phase 2 consisted of a blend of several analytic techniques and procedures developed by Strauss and Corbin (1990), used to develop subcategories, properties and dimensions, as well as generate dozens of possible codes. Through conceptual ordering (classifying events without necessarily identifying relationships), these codes were organized and refined into categories.

Table 11 summarizes the categories, subcategories, and properties that were developed during Phases 1 and 2 of analysis. These resulted from open, axial, and selective coding, and represent the coding system used to integrate and refine the theory. 
- Concept Definition of Tangent Line

○ Subcategory1: Process

- Properties: Ratio, Limit

○ Subcategory2: Object

- Properties: Slope, One Point

○ Subcategory3: Referencing

- Properties: Task 2, Task 3, Task 4

- Concept Image of Tangent Line

○ Subcategory1: Descriptions

- Properties: Location, Slope, Slope Zero, Derivative, Limit, Secant, Concavity Change, Zoom, Straight Line, DNE

○ Subcategory2: Reasoning

- Properties: Definition, Guessing, Rule, Class, Derivative, Slope, Method

- Concept Image of Derivative

○ Subcategory1: Process

- Properties: Ratio, Limit, Function

○ Subcategory2: Object

- Properties: Slope, One Point, Derivative

○ Subcategory3: Descriptions

- Properties: Slope, Slope Graph, Slope Tangent, Slope Zero, Increasing, Decreasing, Constant, Pointy, DNE

- Subcategory4: Reasoning

- Properties: Definition, Guessing, Rule, Class, Shape, Slope, Method

- Cognitive Conflict

○ Subcategory1: Type

- Properties: Concept Definition, Concept Image, Formal Definition

Table 11. Data analysis. Categories, subcategories, and properties.

\subsubsection{Phase 3- theorizing}

Strauss and Corbin (1990) define theorizing as "the act of constructing from data an explanatory scheme that integrates various concepts through statements of relationship" (p. 25). They describe validating theory as a "process of comparing concepts and their relationships against data during the research act to determine how well they stand up to such scrutiny" (p. 24). The StudioCode software was an analytic tool that assisted with theorizing and validating the theory.

I created movie packages for the video data (Interview 3), which consisted of a video file and a timeline (Figure 9). In addition to the movie packages, I also created a code window. This was a visual representation of the codes that were generated and refined during Phases 1 and 2 of data analysis (Figure 9), and that continued to be grouped and refined during this phase as well. 
The codes were used to create instances on the data timeline that could be grouped in various ways to help make sense of the data and organize these into a theoretical explanatory scheme.

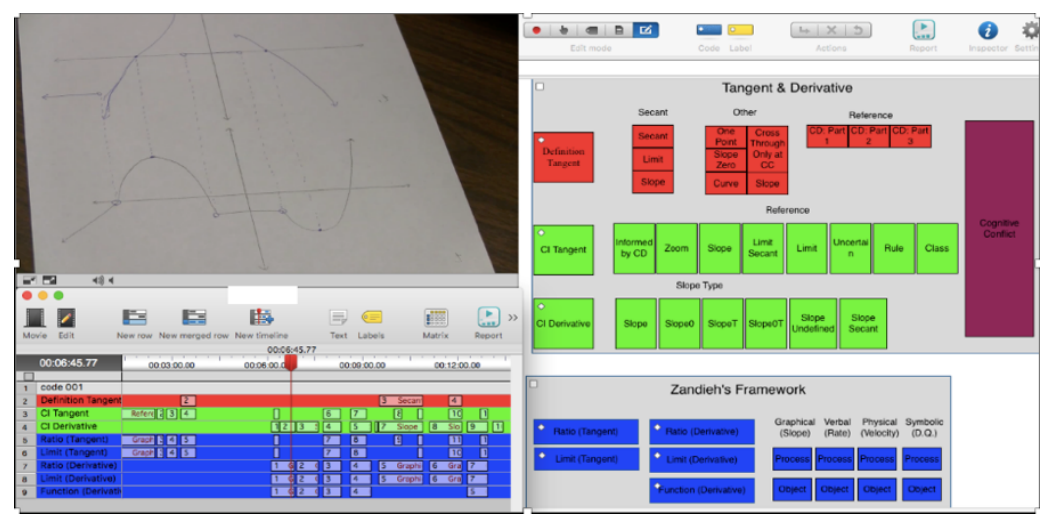

Figure 9. StudioCode- movie package and code window.

\subsubsection{Summary}

Strauss and Corbin's (1990) grounded theory was utilized in my three phases of data analysis described in this chapter. Open, axial, and selective coding were part of each phase of analysis as I cycled through different approaches of looking at the data. At times my approach to analysis was exploratory, at other times, very detailed, and at other times, more conceptual. Phase 1 was mainly exploratory and involved the analytic techniques of diagramming and journaling. Phase 2 was more detailed and included microanalysis, identifying categories, and developing them in terms of properties and dimensions. Phase 3 incorporated a more conceptual approach to analysis for the purpose of making comparisons and discovering relationships. All in all, data analysis was both a calculated and creative process that was guided by the ultimate goal of generating theory grounded in data. 


\section{Chapter 5 - Data}

\subsection{Introduction}

In this chapter, data is presented from all twelve subjects for the series of interviews, with an emphasis on data from Interview 3 of the four core students, Aaron, Colton, Jamie and Alice. Aaron and Colton had both previously taken first-semester calculus, while Jamie and Alice were taking calculus for the first time.

To review, the four tasks of Interview 3 were the following:

Task 1: Definition of a tangent line

Task 2: Construction of tangent lines

Task 3: Sketching the graph of the derivative for a given function graph

Task 4: Graphically interpreting the formal symbolic definition of the derivative

Data for Tasks 1 and 2 pertain to the students' concept definitions and images of the tangent line, and data for Tasks 3 and 4 pertain to their concept images of the derivative. A student's concept definition is regarded as "a form of words used to specify that concept," and a student's concept image is regarded as "the total cognitive structure that is associated with the concept, which includes all the mental pictures and associated properties and processes" (Tall \& Vinner, 1981, p. 152).

In this chapter, the students' concept images of the tangent line and the derivative are described in terms of elements of their understandings that were identified in the data. Elements of a student's concept image of the tangent line are labeled $t E$, and elements of his or her concept image of the derivative are labeled $d E$. For some students, a connecting element, denoted $c E$, was identified in the data. A connecting element is an explanatory statement that links two concept images. It may be thought of as a pathway from one concept image to the next. For example, 
"The derivative is equal to the slope of the tangent line at a point" would be a connecting element from one's concept image of the tangent line to that of the derivative. An expanded concept definition of the tangent line is also presented for each student. This definition was derived from personal concept definitions of the tangent line from Task 1 and identified concept image elements of the tangent line from Task 2. The expanded definition encapsulates the meaning a student associates with the words of his or her concept definition. It also serves as a concise description of a student's understanding for the tangent line.

As one reads the data presented in the following pages, keep in mind that the analysis considers an ideal concept image of the tangent line and derivative to contain the process-object pairs for the layers of the frameworks, since the frameworks represent the formal definitions for the concepts. In reviewing the data, consider how each student's definition of the tangent line aligns with the formal definition, and in turn, how his or her approach to reasoning about the derivative is influenced by this definition. 


\subsection{Aaron}

\subsubsection{Task 1: Concept definition of the tangent line}

The first table of this section, Table 12, shows Aaron's concept definition of the tangent line for the series of interviews.

\begin{tabular}{|l|l|}
\hline Aaron & Concept definition of the tangent line \\
\hline Interview 1 & $\begin{array}{l}\text { A tangent line is a line that intersects a function. Well, it touches, just briefly touches it } \\
\text { at one point, and it's involved where you find the, you differentiate a function, you find } \\
\text { the derivative of it, it's used in that ... It represents the um the slope of a function at a } \\
\text { single point .... As opposed to like a secant line which will be the average, it would } \\
\text { cross two points. That would be like the average slope between two points. That will be } \\
\text { the secant line. It's different than the secant. }\end{array}$ \\
\hline Interview 2 & $\begin{array}{l}\text { A tangent line would be a representation of the slope at a certain point. It would just } \\
\text { follow, you pick a certain point, like there, and then you would trace a line, and that } \\
\text { would represent the slope narrowing it down to that point right there. }\end{array}$ \\
\hline Interview 3 & $\begin{array}{l}\text { It is the line that represents the, it has a slope of a function at a certain point. As } \\
\text { opposed to a secant line, where it's like connecting two points on a function and is the } \\
\text { line that goes in between them. As the second point approaches the first point, it's like } \\
\text { the limit of that. And that's the tangent line. It represents the slope. }\end{array}$ \\
\hline
\end{tabular}

Table 12. Aaron. Task 1. Summary chart.

During Interview 1, one can almost see different elements of his concept image being activated and assisting in the construction of his concept definition. By Interview 3, he had a more concise and comprehensive definition.

Aaron mentioned slope and one point (or certain point) in each of his definitions. These were common phrases used for defining the tangent line among all twelve participants. "Slope" and "one point" are objects for the layers of the Tangent Line Framework. In terms of processes, Aaron did not describe a process for calculating slope in his definition, but did demonstrate an understanding for this process at other times in his interviews. For example, during Task 4 he referenced change in $y$ over change in $x$ as a formula for calculating slope. Thus, slope was not a pseudo-object for Aaron. Likewise, “one point," was not a pseudo-object element of his concept definition, since during Interview 3, he described the underlying limit process for this object. 
Aaron mentioned the limit process in his definition for the third interview, but not for the first two interviews. In this way, it may appear that his understanding of the tangent line underwent major changes. However, this was not the case. Although the limit process was not included in his concept definition, he did reference this process when constructing tangent lines during the first interview:

"You can't know at a single point. You're always having to... it'd be like $\frac{y_{2}-y_{1}}{x_{2}-x_{1}}$. And, you don't want to get zero in the denominator. So, it's like a limit. You're approaching that, this distance $\left[x_{2}-x_{1}\right]$ is getting smaller and smaller here, and then, once this distance is closed, you end up with just like one point there, and that's how you end up with the tangent line.” (Interview 1, Task 2)

The limit process was part of his understanding of the tangent line from the first interview but was only included in his concept definition of the tangent line during the third interview. This point is highlighted to note that his concept image of the tangent line was not necessarily undergoing any changes, but rather, the aspects of his concept image that he chose to include in his concept definition were more process-focused by the third interview.

\subsubsection{Task 2: Construction of tangent lines- six graphs}

The next table summarizes Aaron's tangent line constructions for the six graphs of Task 2. The table also shows excerpts from his Interview 3 transcript for some of the graphs. The quotes highlight his ways of thinking about constructing tangent lines or the tangent line not existing. 


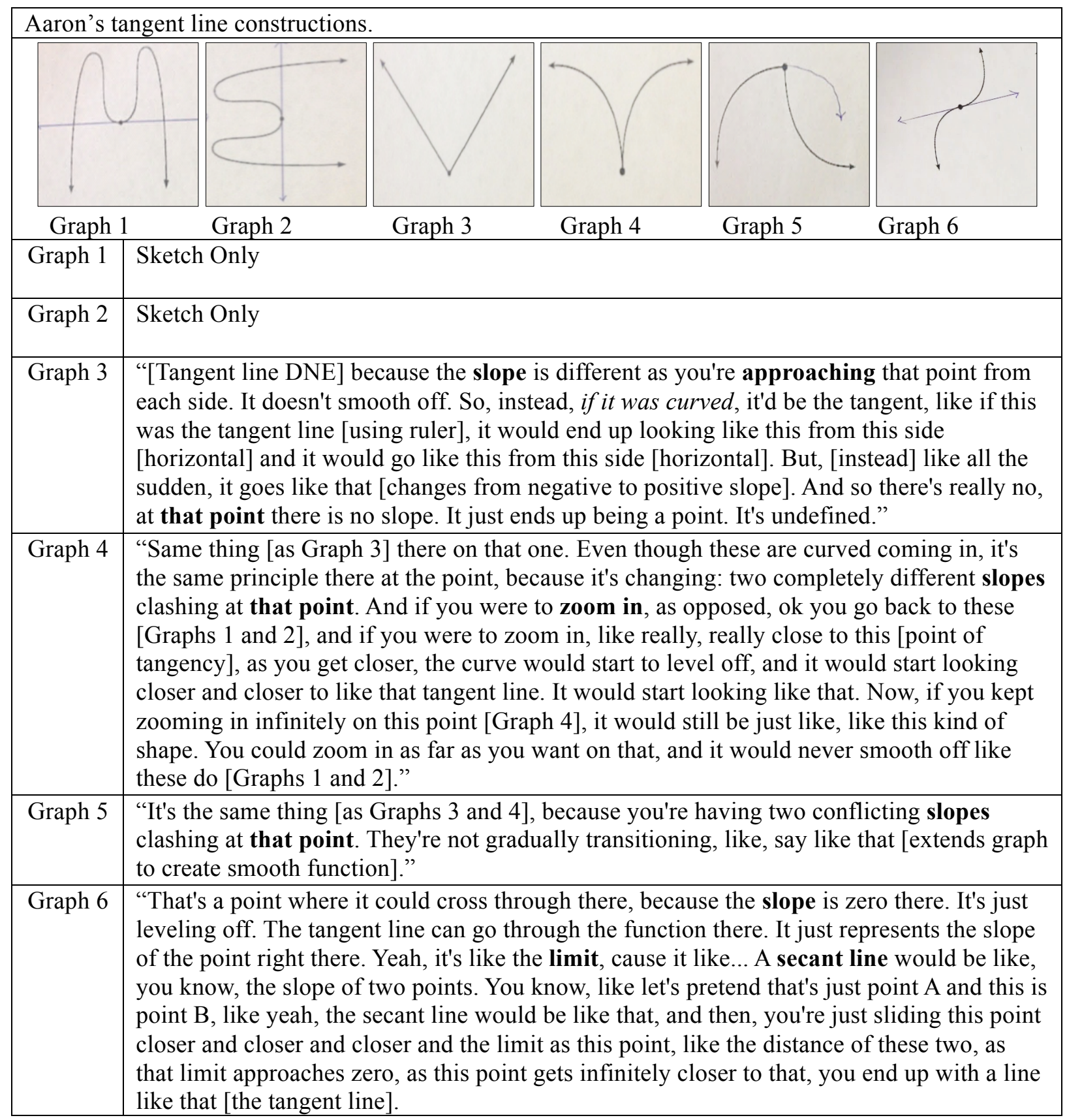

Table 13. Aaron. Task 2, Interview 3. Summary chart.

The quotes in the table represent Aaron's descriptions for what was being activated in his concept image in response to the sketching tasks. Notice that his language for describing the tangent line during Task 2 and defining the tangent line from Task 1 are very similar. Slope, one point, secant line and limit were emphasized elements of both his concept definition and image. 
To this extent, it is evident that his concept image and definition were informed by one another, and his reasoning flowed through both of these cells.

An important feature of Aaron's data was that he consulted his concept definition of the tangent line when reasoning about tangent lines during Task 2. For example, consider his response during Graph 6, when he said, "you're just sliding this point closer and closer and closer and the limit as this point, like the distance of these two, as that limit approaches zero, as this point gets infinitely closer to that, you end up with a line like that [the tangent line]." This directly corresponds to his definition of the tangent line from Task 1, which included, "As the second point approaches the first point, it's like the limit of that." These demonstrate that Aaron recognized the limit process as a defining feature of the tangent line and evoked this understanding in order to explain the slope of his tangent line sketches. In this way, the limit process was a prioritized element of his concept image, as he was able to easily access this knowledge in necessary settings. This feature of his data distinguished him from the remaining eleven participants of this study.

Aaron mentioned the idea of magnification, or zooming in, during his explanation for Graph 4. This feature of his understanding also distinguished him from his peers in this study. Although, to be clear, one student did imagine zooming in on a graph by using her two fingers to enlarge the graph as if on an iPhone. However, this was in order to demonstrate a one-point property with the curve, not local linearity. The principle of local linearity is closely related to that of differentiability. By mentioning the visual, geometric aspect of local linearity, Aaron revealed that his concept images for the tangent line and derivative were closely connected. However, at this point in the interview, he had not yet specified a relationship between the concepts. 
Based on Aaron's data from Tasks 1 and 2, elements of his concept image of the tangent line were identified (Table 14). These elements summarize the evoked portions of his concept image during the tasks. Considering these elements in conjunction with how he defined the tangent line, an expanded concept definition was derived. The expanded definition encapsulates the meaning of tangency that Aaron conveyed.

\begin{tabular}{|c|c|}
\hline Aaron & Tangent Lines (Tasks 1 and 2) \\
\hline $\begin{array}{l}\text { Concept } \\
\text { Definition }\end{array}$ & $\begin{array}{l}\text { It is the line that represents the, it has a slope of a function at a certain point. As } \\
\text { opposed to a secant line, where it's like connecting two points on a function and is } \\
\text { the line that goes in between them. As the second point approaches the first point, } \\
\text { it's like the limit of that. And that's the tangent line. It represents the slope. }\end{array}$ \\
\hline $\begin{array}{l}\text { Elements of } \\
\text { Concept Image }\end{array}$ & $\begin{array}{l}\text { tE1: A secant line is a line connecting two points on a function graph. } \\
\mathrm{tE} 2: \text { The tangent line is the limiting position of secant lines. } \\
\text { fE3: For the tangent line to exist at a point, the limit of the slopes of the function } \\
\text { tEx: The tangent line represents the slope of the graph at a certain point } \\
\text { tE5: If the tangent line exists at a point, then under magnification, the curve should } \\
\text { start to level off and look closer and closer to the tangent line around the point of } \\
\text { tangency. If the graph does not smooth off around the point of tangency, then the } \\
\text { tangent line does not exist at that point. }\end{array}$ \\
\hline $\begin{array}{l}\text { Expanded } \\
\text { Concept } \\
\text { Definition }\end{array}$ & $\begin{array}{l}\text { The tangent line is the limiting position of secant lines whose slope is representative } \\
\text { of the slope of the function at a certain point, and at this point, under magnification, } \\
\text { the tangent line and the graph look the same. }\end{array}$ \\
\hline
\end{tabular}

Table 14. Aaron. Tasks 1 and 2, Interview 3. Concept definition and image of tangent line.

\subsubsection{Task 3: Sketching the graph of $f^{\prime}(x)$, given the graph of $f(x)$}

The next task Aaron completed during Interviews 2 and 3 was sketching the graph of the derivative function for a given function graph, for which the formula was not provided. Table 15 summarizes his work for this task on Interview 3. The graphs shown in the table are replicas of Aaron's original sketch, and the labels on the $x$-axis were not part of his (or any of the students) original sketches but were added in order to describe the data. The quotes in the table represent his descriptions for the graph of the derivative on the indicated intervals. 


\begin{tabular}{|c|c|c|}
\hline Aaron & $\begin{array}{l}\text { Original function } \\
\text { graph }\end{array}$ & $\begin{array}{l}\text { Aaron's sketch of } \\
\text { derivative }\end{array}$ \\
\hline $\begin{array}{l}x=a: \\
\text { "You find the uh points where the slope is zero, the } \\
\text { slope of the tangent line is zero ... And then, I just } \\
\text { draw these points down ... put these points here at } \\
\text { zero." } \\
x=b, c \text { : } \\
\text { "And so the points where it's not as smooth, you } \\
\text { know, it's basically where it's like a point, those are } \\
\text { where the uh, the slope would be undefined. So, } \\
\text { there's open points there ... It's like two conflicting } \\
\text { slopes like meeting from each side." } \\
\text { Interval }(-\infty, a) \text { : } \\
\text { "Here the slope is positive and as you're approaching } \\
\text { this, you know, it goes towards zero ... the slope } \\
\text { over here entirely, like the slope is positive, but it's } \\
\text { becoming less positive as you go toward that point, } \\
\text { so, it's going down toward zero [on the graph of the } \\
\text { derivative]. } \\
\text { Interval }(a, b) \text { : } \\
\text { "And then, as you go past that it becomes negative so } \\
\text { it's going like this." } \\
\text { Interval }(b, c] \text { : }\end{array}$ & & \\
\hline $\begin{array}{l}\text { Interval }(c, d] \text { : } \\
\text { "Here, the slope is very negative. So, you're going to } \\
\text { go up until you have a slope of zero right there." } \\
\text { Interval }(d, e) \text { : } \\
\text { "And this is heading on up. Here [on the graph of the } \\
\text { derivative], it's just getting very, very positive and } \\
\text { keeps going up." } \\
\text { Interval }(e, \infty) \text { : } \\
\text { "And then, you run into a constant slope here. It's } \\
\text { just, you know, a straight line." }\end{array}$ & & \\
\hline
\end{tabular}

Table 15. Aaron. Task 3, Interview 3. Summary chart.

His sketch was mainly correct except for thinking that the derivative approached negative infinity to the right of $c$ and positive infinity to the left of $e$. It is fair to mention that he did not make this same mistake during Interview 2. For this interview, his sketch of the derivative was completely correct. 
Comparing Aaron's data for constructing tangent lines to that of sketching the derivative function, we see that he used similar language for these two tasks. For Graphs 3, 4, and 5 of Task 2, he said the tangent line would not exist at indicated points because of "undefined," "clashing," and "conflicting" slopes. Similar reasoning was demonstrated during Task 3, when he said the derivative would have "open points" for $x=b$ and $x=c$ since the original function had "undefined" and "conflicting" slopes at these places. The majority of participants of this study (ten of twelve) used open circles in their derivative sketches, not all did so correctly, however, for these undefined values, they often reasoned in terms of the shape of the graph- it being "pointy"- and not in terms of the slope of the graph, like Aaron.

Aaron's responses in the above table indicate that he connected the notion of slope to that of the derivative, and in one instance, $x=a$, he specified that slope to be the slope of the tangent line. In order to clarify how Aaron was determining the value for the derivative function, the interviewer asked him to discuss the meaning of the $y$-value for an indicated point on $f$ prime. He said,

"That is the um, the slope. The steepness of the slope. Like, let's say, yeah, like there [pointing to $y$-value on $f$ prime], that would be like negative one or something, so the [original] function at that point would be... it would have a slope... the tangent, the slope of the tangent line would be negative one at that point."

This was the first time in Aaron's interview that he described a relationship between the tangent line and the derivative. In fact, this was only the second time that he referenced the notions together, the first was at $x=a$ in the above table. Although his descriptions of the tangent line from Tasks 1 and 2 were prime for making connections to the derivative, he did not reference the derivative during these tasks. This was an interesting feature of his interview, and 
in many ways, distinguished him from the other participants, who often quickly associated the two notions. Some students referenced the derivative as early as Task 1, as part of their definition of a tangent line. These same students were unable to describe a relationship for the concepts in the context of Task 3. Other students often referenced the derivative during Task 2, in order to explain why the tangent line did not exist at corner points. However, their explanations often lacked meaning, such as, "I remember from class" or "Because the graph comes to a point." On the other hand, Aaron did not reference the derivative during Task 1 or 2, but instead, he reasoned about the tangent line in terms slope, limit process, magnification, secant lines, and the behavior of the graph near the point of tangency. This showed that his understanding of the tangent line was robust enough to include descriptions beyond associating it with the derivative in a meaningless way.

At the conclusion of Task 3, the question still remained as to what level of detail Aaron could provide in explaining his claim that the derivative was equal to the slope of the tangent line at a point. It was relevant to consider if this connection was pseudostructural in nature or if he was able to unpack this idea and describe the underlying processes. The data in the next section explores these aspects of his concept image.

\subsubsection{Task 4: Graphically interpreting the formal symbolic definition of the derivative}

Upon seeing the symbols that define the derivative function, Aaron quickly recognized, "you're just building on concepts." The limit symbol quickly activated the limit process in his concept definition of the tangent line and he pieced the two concepts together in a way that surpassed the ability demonstrated by the other eleven participants of this study, none of which had a concept definition of the tangent line that included a limit process.

Table 16 on the next page summarizes Aaron's data for Task 4. 


\begin{tabular}{|l|}
\hline \multicolumn{1}{|c|}{ Aaron's graphical interpretation of the formal definition of the derivative. } \\
\hline "I mean, it's similar to um, if you were to have, \\
again you're just building on concepts. Like, if \\
you were to have a secant line, it would be like, \\
you have points A, and then that's coordinates \\
$\left(x_{1}, y_{1}\right)$ and then another one up here would be \\
coordinates $\left(x_{2}, y_{2}\right)$. And, it's basically the same \\
concept as this [pointing to $\frac{f(x+h)-f(x)}{h}$ of the \\
definition], [which] represents the slope. We're \\
not quite to the tangent-line-derivative-thing yet \\
$\ldots$. It would be basically like $\frac{y_{2}-y_{1}}{x_{2}-x_{1}} \ldots$. You're \\
closing it in and as that distance, $h$, as you're \\
moving this point towards the other point, um the \\
$h$ grows smaller. And, as it approaches, the \\
distance approaches zero. As you're getting \\
infinitely closer to that [other point], this number \\
[circling $h$ ] keeps getting smaller and smaller and \\
towards zero. And then, you would find, you'd \\
solve that and then you would find the slope of \\
the tangent line at that point, or the derivative."
\end{tabular}

Table 16. Aaron. Task 4, Interview 3. Summary chart.

Note that Aaron referenced the process-object pairs for the first two layers of the

Derivative Framework. He correctly interpreted the difference quotient to be the slope of a secant line (object, layer one), and then, described a process for calculating slope, $\frac{y_{2}-y_{1}}{x_{2}-x_{1}}$

(process, layer one). He correctly described the limit process (process, layer two), and explained that it resulted in the slope of the tangent line (object, layer two).

A bit later in the interview, he discussed the function process, the third layer of the framework. The function process involves running through every point on the original function graph and extracting the instantaneous slope value. The object of the third layer is the derivative function. The following quote from Aaron's third interview highlights his knowledge of this layer:

"Like, let's put $3 x^{2}+2$ or something as the original function, and then, you would find the um derivative of that, and then, you would plug in an $x$-value there and that would be, 
into the derivative, the derivative function [pointing to emphasize the derivative function, $n o t$ the original function], and that would find the um slope of the tangent line at that $x$ point."

In conclusion, through analysis of Aaron's data for Tasks 3 and 4, seven elements of his concept image of the derivative were identified. A connecting element between his concept image of the tangent line and the derivative was also identified. The details of a connecting element were discussed in the Introduction to this chapter, but to review, a connecting element may be thought of as a pathway from one concept image to another. It is a statement that describes a relationship between two concepts. The identified elements of Aaron's concept image of the derivative and the connecting element are summarized in Table 17 on the next page. 


\begin{tabular}{|l|l|}
\hline Aaron & \multicolumn{1}{c|}{ Derivative (Tasks 3 and 4) } \\
\hline Elements & $\begin{array}{l}\text { dE1: The value of the derivative is equal to zero when the tangent line has a slope of } \\
\text { zero. } \\
\text { dE2: At places where } f(x) \text { is not smooth, the slope is undefined. At these places, the } \\
\text { graph of } f^{\prime}(x) \text { will have open circles. } \\
\text { dE3: The graph of the derivative goes up, down, or straight on a given interval based } \\
\text { on the behavior of the slopes of the graph of } f(x) \text { on the interval. } \\
\text { dE4: For a given point on the derivative graph, }\left(x, f^{\prime}(x)\right) \text {, the } y \text {-value represents the } \\
\text { slope of the tangent line at }(x, f(x)) \\
\text { dE5: The ratio of the definition of the derivative, } \frac{f(x+h)-f(x)}{h}, \text { is equivalent to } \frac{y_{2}-y_{1}}{x_{2}-x_{1}}, \\
\text { which represents the slope of the secant line through the points }\left(x_{1}, y_{1}\right) \text { and }\left(x_{2}, y_{2}\right) \\
\text { on the original graph. } \\
\text { dE6: As the distance between the two points that determine the secant line approach } \\
\text { zero, the secant lines approach the tangent line, and the limit of the slopes is equal to } \\
\text { the slope of the tangent line. } \\
\text { dE7: For a given } x \text {-value, the derivative function finds the slope of the tangent line at } \\
\text { that } x \text { point on the original function }\end{array}$ \\
$\begin{array}{l}\text { cE1: For any } x_{o} \in d o m f^{\prime}(x), f^{\prime}\left(x_{o}\right) \text { is equal to the slope of the tangent line at } \\
\left(x_{o}, f\left(x_{o}\right)\right) .\end{array}$ \\
\hline Elemecting
\end{tabular}

Table 17. Aaron. Tasks 3 and 4, Interview 3. Concept image of the derivative.

Aaron correctly described the graphical meaning for the symbols of the derivative function by accessing the limit process of his concept definition of the tangent line. The three remaining students discussed in this chapter did not define the tangent line in terms of a limit process, and they also struggled to identify the graphical meaning for the symbols of the derivative function in Task 4. In fact, the other eight participants of this study, also did not define the tangent line in terms of a limit process, and like Alice, Colton, and Jamie, also experienced difficulty on Task 4. One of the eight students was able to interpret the graphical meaning of the definition, but required a lot of prompting and was not able to provide as detailed of a description as Aaron. Table 18 below reviews Tasks 1 and 4 for these eight students. Notice the difficulty each student had in understanding the limit process for the definition of the derivativespecifically, the meaning of $h$. While looking at their data, keep in mind that Aaron defined the 
tangnet line in terms of a limit process and was able to quickly activate this during Task 4 . These

students, on the other hand, defined the tangent line in terms of pseudo-objects, not processes.

\begin{tabular}{|c|c|c|}
\hline \multicolumn{3}{|c|}{ Concept definition of the tangent line compared to graphical understaning of the derivative } \\
\hline Student & $\begin{array}{l}\text { Task 1- Concept definition of the tangent } \\
\text { line }\end{array}$ & $\begin{array}{l}\text { Task 4- Graphical interpretation of the formal } \\
\text { symbolic definiton of the derivative. }\end{array}$ \\
\hline Amanda & $\begin{array}{l}\text { "A line that passes through any point on } \\
\text { the curve but it has to like at that specific } \\
\text { time." }\end{array}$ & $\begin{array}{l}\text { "I've not thought about [what } h \text { means]. I've } \\
\text { always just done it... Would I be allowed to } \\
\text { use derivative rules to prove this is the } \\
\text { derivative? }\end{array}$ \\
\hline Anita & $\begin{array}{l}\text { "It's the slope of the secant line. It only } \\
\text { touches the graph at one point." }\end{array}$ & " $h$ is like, I don’t really know what $h$ is." \\
\hline Carly & $\begin{array}{l}\text { "A tangent line is the same as the } \\
\text { derivative at a point on a function" }\end{array}$ & $\begin{array}{l}\text { "I don't know how this (sketching the } \\
\text { derivative graph, Task } 3 \text { ) applies to this } \\
\text { (symbolic defintion, Task 4)...I wonder what } h \\
\text { is." }\end{array}$ \\
\hline Corey & $\begin{array}{l}\text { "It is a line that will show the } \\
\text { instantaneous rate of change at a point on } \\
\text { a graph." }\end{array}$ & $\begin{array}{l}\text { "I don't know [what } h \text { means]. Maybe I knew } \\
\text { when we first learned it." }\end{array}$ \\
\hline Jacob & $\begin{array}{l}\text { "A line that represents the slope of the } \\
\text { graph at a particular point." }\end{array}$ & $\begin{array}{l}\text { "I guess } h \text { would kind of represent, as the gap } \\
\text { between these two numbers gets smaller and } \\
\text { smaller." [circling } y_{2}-y_{1} \text { of the slope formula } \\
\text { he had written on his paper ]." }\end{array}$ \\
\hline Kevin & $\begin{array}{l}\text { "It skims the function. It can pass } \\
\text { through but it usually doesn't." }\end{array}$ & "I've never thought about that [what $h$ means]" \\
\hline Kenneth & $\begin{array}{l}\text { "The line parallel to the point that's } \\
\text { given, if there is one." }\end{array}$ & $\begin{array}{l}\text { "I don't really even know } h \text {. I mean, it's } \\
\text { approaching zero, but I only take it as a place } \\
\text { holder for the definition of derivative. That's } \\
\text { really only what I think of. " }\end{array}$ \\
\hline Mindy & $\begin{array}{l}\text { "A tangent line is the slope of a point, } \\
\text { like one point." }\end{array}$ & $\begin{array}{l}\text { "I probably wouldn't know what } h \text { is } \\
\text { [graphically]. That's pretty bad." }\end{array}$ \\
\hline
\end{tabular}

Table 18. Remaining eight participants. Tasks 1 and 4, Interview 3. 


\subsection{Colton}

\subsubsection{Task 1: Concept definition of the tangent line}

The first table of this section summarizes Colton's concept definition of the tangent line for the three interviews of the series. In his definitions, notice that Colton consistently referenced slope, one point, and a curve.

\begin{tabular}{|l|l|}
\hline Colton & Concept definition of the tangent line \\
\hline Interview 1 & The slope of a curve at a single point \\
\hline Interview 2 & The line that touches a curve at one point and has its slope at that point. \\
\hline Interview 3 & $\begin{array}{l}\text { A line or a plane that touches a curve at one spot and if you continue, won't touch it } \\
\text { again. }\end{array}$ \\
\hline
\end{tabular}

Table 19. Colton. Task 1. Summary chart.

Comparing Colton's concept definition of the tangent line to the Tangent Line

Framework, the two overlap only in terms of objects. He defined the tangent line in terms of slope and one point- the objects of the first two layers. However, he did not describe the ratio or limit processes of these two layers.

Colton's emphasis on a curve was an interesting aspect of his concept definition. In fact, the requirement for a curve narrowed his understanding of the tangent line. To quickly illustrate, he did not think the tangent line existed for any point on a linear function since these are "straight lines" and "there's no curve." This aspect of his concept definition caused him cognitive conflict during Task 3 when activated in conjunction with his definition of the derivative. The details of this conflict will be elaborated on in later sections, but it was brought about by his incorrect definition of the tangent line.

\subsubsection{Task 2: Construction of tangent lines- six graphs}

The second interview task considered how Colton constructed tangent lines and the data discussed is representative of what belonged to his concept image of the tangent line. For this task, Colton was presented with six graphs and was asked to sketch the tangent line at each 
indicated point of tangency. The following table shows his tangent line sketches for the six graphs and also transcript excerpts that highlight his thought processes.

\begin{tabular}{|l|l|}
\hline Colton's tangent line constructions \\
\hline Graph 1 & "That one's a tangent of zero. It's a concavity change, or it's a um minimum there." \\
\hline Graph 2 & $\begin{array}{l}\text { "And uh same goes for this one as well. Oh, that wouldn't be a function, so it only touches } \\
\text { the curve in one area." }\end{array}$ \\
\hline Graph 3 & "These two are not because they come to points [Graphs 3 and 4]." \\
\hline Graph 4 & "These two are not because they come to points [Graphs 3 and 4]." \\
\hline Graph 5 & $\begin{array}{l}\text { "And then this one's the same story [as Graphs } 3 \text { and 4]. It comes to a point there and } \\
\text { changes [concavity]. So there was no curve." }\end{array}$ \\
\hline Graph 6 & $\begin{array}{l}\text { "And then this one you could, but it's changing [concavity] there, so it's the end of, uh, it's } \\
\text { a concavity change on these two." } \\
\text { "Not sure. Still after the third one [interview], I'm still not sure. But I think that should be } \\
\text { ok, because you're, it would still be at the end. If you separated them, it would still be at the } \\
\text { end of this one [curve], so you could still zero that out and then that one." }\end{array}$ \\
\hline
\end{tabular}

Table 20. Colton. Task 2, Interview 3. Summary chart.

In analyzing Colton's responses for the Six Graphs, two aspects of his concept image of the tangent line called for consideration:

1) In his concept definition during Task 1 , he described a tangent line as having one point in common with the curve. However, his tangent line sketches in Task 2 have multiple points in common with the graph.

2) He often referenced concavity changes of the graph, but how was he relating this to the existence of the tangent line?

Data related to each of these points is considered next.

First, for Graph 1, Colton sketched a tangent line that had three intersection points with the graph. However, his concept definition from Task 1 seemed to restrict a tangent line to having only one point in common with the curve. When asked about this discrepancy by the 
interviewer, Colton explained that it was ok for there to be multiple intersecting points "because you've changed concavity" and "your curve is not the same as this curve here."

When he said "you've changed concavity" he did not mean at the point of tangency but that the graph as a whole had places where the concavity changed. He thought these concavity changes divided the graph into several curves. Figure 10 is my depiction of how Colton may see the graph divided in his mind.

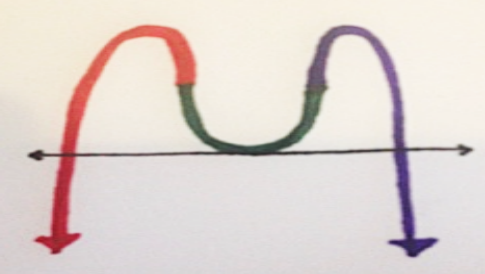

Figure 10. Colton. Multiple curves.

The figure can be used to demonstrate what he meant when he said, "your curve [red or purple] is not the same as this curve here [green]." Meaning, the tangent line he constructed for Graph 1, was only touching the curve for which the point of tangency was located [green] only once. By breaking the graph into several curves based on concavity changes, Colton was able to stay true to his concept definition of the tangent line as a line touching the curve in only one spot.

Second, for Graphs 3, 4, and 5, Colton correctly did not sketch a tangent line since these points were located at corner points of the graphs. When asked to explain this decision, he referred to his concept definition, and stated, "It's not a curve." The interviewer pressed about why the presence of a curve would be necessary for the tangent line to exist. He continued with his same line of thinking: "You'll just intersect there. It won't be a tangent line, because for it to be tangent, there has to be a curve." Ultimately, he reasoned that it was "part of the definition [of 
a tangent line]." It is interesting that he said "the definition." This insinuated that he regarded his personal concept definition to be the formal definition of the concept.

In addition to referencing his concept definition, Colton also explained that the tangent line would not exist at corner points, because "you can't derive a point." He used this phrase throughout his interview, and examining these instances in the data, it seems by 'can't derive a point," Colton meant at corner points or cusps of the original function, the derivative does not exist. And by using this phrase in the context of sketching the tangent line, he evidenced hat he connected the notions of tangent line and derivative. In particular, he evidenced a belief that, if the derivative does not exist for a certain point on a function graph, then the tangent line does not exist at that point either. This line of thinking was common among participants of the study. For example, Alice, who is discussed later in this chapter, also reasoned the tangent line would not exist for Graphs 3 and 4 based on the shape of the graph (coming to a point) and the derivative not existing.

Although Colton related the non-existence of the derivative and tangent line, he was not quite sure of the details of this statement in the context of the task. When asked, he said, "Because the concavities change," which implied a belief that the derivative does not exist when the graph changes concavity. Beyond this isolated quote, there was not data available to further clarify this feature of his understanding. However, there was available data for describing how Colton connected concavity changes in the graph to the tangent line not existing. The next set of quotes are related to graphs for which Colton believed the tangent line would not exist. Notice his references to concavity change:

"It comes to a point there and changes [concavity]."

"The concavities change. You can't derive it there." 
Also, for Graph 6, the point of tangency was located at a concavity change in the graph. Colton very hesitantly constructed a tangent line at this point. He did so correctly, but was uncertain about his decision. He said, "Not sure. Still after the third one [interview], I'm still not sure. But I think that should be ok." He thought the tangent line may not exist because of the concavity change in the graph. However, he was conflicted because the concavity change did not occur at a corner point of the graph. Since the graph did not come to a sharp point, he reasoned that the derivative would exist at this concavity change. He then reasoned that if the derivative existed so should the tangent line. Essentially, he had two conflicting lines of thought and was experiencing some cognitive conflict. He was trying to remedy the following elements of his concept image- 1.) There's a concavity change in the graph, so the tangent line should not exist. 2.) The derivative exists (since the graph does not come to a sharp point), so the tangent line should exist.

To convince himself that the tangent line he sketched was correct, he separated the graph into two curves at the concavity change, just like he had done for Graph 1, and reasoned:

"If you separated them, it would still be at the end of this one [curve], so you could still zero that out and then that one. But, it would be an inflection change, so. Um, well if you did it, your slope would approach zero there. So, I guess that's ok. If you took like, if you're looking at it from the limit perspective. As it changes it went down. It would zero out there, so."

To clarify, when he said "the limit perspective," he meant the limit of tangent lines "surfing" towards the point of tangency. He was using a ruler to represent the tangent line and was moving the ruler along the curve to watch the slopes approach zero at the point of tangency. 
In light of his tangent line sketch for Graph 6, he adjusted his concept definition. This new definition included an exception concerning concavity changes in the graph.

"A line or a plane that intersects with a curve at one point and won't cross through that [the graph] unless there's a concavity change."

From Tasks 1 and 2, five elements of Colton's concept image of the tangent line were identified in the data. These are listed in Table 21 on the next page, along with an expanded version of his concept definition of the tangent line, derived from the data. It is interesting to point out that, like his concept definition, Colton's concept image of the tangent aligned with the framework in terms of only objects. He did not make references to the ratio or limit processes of the formal definition. 


\begin{tabular}{|l|l|}
\hline Colton & \multicolumn{1}{c|}{ Tangent Lines (Tasks 1 and 2) } \\
\hline $\begin{array}{l}\text { Concept } \\
\text { Definition }\end{array}$ & $\begin{array}{l}\text { A line or a plane that touches a curve at one spot, and if you continue, won't touch it } \\
\text { again. }\end{array}$ \\
\hline $\begin{array}{l}\text { Clements of } \\
\text { tE1: For a tangent line to exist, there must be a curve; tangent lines do not exist } \\
\text { when the graph is a straight line or comes to a sharp point. } \\
\text { tE2: Concavity changes break the graph up into several curves. The tangent line will } \\
\text { touch the curve which contains the point of tangency only once. } \\
\text { tE3: In general, the tangent line does not exist at a concavity change in the graph. }\end{array}$ \\
$\begin{array}{l}\text { However, if the graph can be separated into two curves at the concavity change, } \\
\text { such that the tangent lines at the end of each of these curves have the same slope, } \\
\text { then the tangent line does exist at the concavity change. } \\
\text { tE4: The tangent line can cross through the curve at the point of tangency only if } \\
\text { there is a concavity change. } \\
\text { tE5: The derivative and the tangent line are connected, in that if the derivative } \\
\text { exists, so does the tangent line, and if the derivative does not exist, then neither } \\
\text { does the tangent line. }\end{array}$ \\
\hline $\begin{array}{l}\text { Expanded } \\
\text { Concept } \\
\text { Definition }\end{array}$ & $\begin{array}{l}\text { A tangent line touches the curve on the graph that contains the point of tangency at } \\
\text { only one spot, and if you continue, the tangent line will not touch that curve of the } \\
\text { graph again, but may intersect the other curves that make up the graph. }\end{array}$ \\
\hline
\end{tabular}

Table 21. Colton. Tasks 1 and 2, Interview 3. Concept definition and image of tangent line.

\subsubsection{Tasks 3: Sketching the graph of $f^{\prime}(x)$, given the graph of $f(x)$.}

The next task Colton completed was sketching the graph of the derivative function for a given function graph, for which the formula was not provided. Table 22 summarizes his work for this task. The quotes are representative of his justifications for each portion of the graph shown. 


\begin{tabular}{|l|l|}
\hline Colton & $\begin{array}{l}\text { Original function } \\
\text { graph }\end{array}$ \\
\hline $\begin{array}{l}\text { Interval }(-\infty, c) \\
\text { "From here }[(-\infty, a)], \text { you're all positive [slope] and } \\
\text { it's getting progressively less positive. The slope's } \\
\text { getting closer to zero at that point }[x=a] \text {. So, we're } \\
\text { going from extremely positive to less positive until } \\
\text { we hit zero there. And then, we'll uh, the slope will } \\
\text { be negative }[(a, b)] \text {. So, then we'll go negative until } \\
\text { here, I suppose }[x=b] \text {. And then, we'll have a slope } \\
\text { of zero because we're not going either way [(b, } c)] .\end{array}$ \\
\hline $\begin{array}{l}\text { Interval }[c, \infty): \\
\text { For here [interval }(c, d)], \text { you're going negative again } \\
\text { and getting progressively less negative. So, it'll be } \\
\text { discontinuous again [at } x=c] \text {. It'll start somewhere } \\
{[\text { degative] and be progressively less [negative] until }}\end{array}$ \\
$\begin{array}{l}\text { we get to here }[x=d] . \text { Fill that in }\left[f^{\prime}(x)=0 \text { at } x=\right. \\
d] . \text { And then, it }\left[f^{\prime}(x)\right] \text { will go positive again, so it } \\
\text { will be going up infinitely 'cause it looks like, well, } \\
\text { not infinitely, but yeah, till we go to there. And then, } \\
\text { because this }[\text { the graph of } f(x) \text { on }(e, \infty)] \text { is not } \\
\text { changing, we'll find whatever that slope is, say a } \\
\left.\text { slope of three, put a three [on the } y \text {-axis of } f^{\prime}(x)\right] \text { and } \\
\text { then we'll just go straight across like that. }\end{array}$
\end{tabular}

Table 22. Colton. Task 3, Interview 3. Summary chart.

Colton reasoned in terms of the slope of the graph. At this point, it was unclear what

Colton meant by "slope," but he later clarified that he thought of the derivative as the slope of the tangent line.

For $x=c$, he described the graph as being discontinuous. This point, along with $x=b$ and $x=e$, caused him a bit of confusion. He could not decide if the graph of the derivative should have open or filled in circles at these points: "I mean, one of them will be filled in, I guess. I don't know how you would determine which one will be filled in from this [the graph of $f(x)]$." In order to make this decision, he concluded that the symbolic formula for $f(x)$ was necessary: "Like if it was, if you had like a piecewise function. That's what I'm thinking of now is a piecewise function. So, I'm just saying that, you know, if that was less than or equal to, that one would be filled in, or if it was, you know, it if was equal to that, then it would have a solid 
dot." Towards the end of the sketching task, he changed his mind about filling in one of the circles and determined, "you'll have two open points at this, because that won't, you can't derive that." He justified this decision stating, "You're changing at that point when the function switched there" and "you won't have a tangent line." It is interesting that in a curve sketching situation it took Colton a while to recall that the derivative does not exist at a corner point, whereas during Task 2, when sketching tangent lines, he quickly activated this knowledge.

The graph of $f(x)$ had constant slope on intervals $(b, c)$ and $(e, \infty)$. On these intervals, he gave the derivative function values of zero and three, respectively. He based his decisions on the shape of the graph of $f(x)$ : for interval $(b, c)$ the graph was "not going either way" and for interval $(e, \infty)$ the graph was "not changing." Once he completed his sketch of $f^{\prime}(x)$, he realized that he had conflicting ideas concerning the decisions he made. The following transcript excerpt captures the development of this realization and highlights the cognitive conflict that he experienced.

Colton: $\quad$ Cause now, you're going completely flat and you can't derive a straight line.

Interviewer: So, there's not a derivative here [pointing to interval $(b, c)$ on $\left.f^{\prime}(x)\right]$ ?

Colton: Right. I don't think you'll have a derivative there cause you're a straight line [graph of $f(x)$ ].

Interviewer: So, this portion of your graph would need to go away? [pointing to interval $(b, c)$ on $\left.f^{\prime}(x)\right]$ ?

Colton: Ok. I'm trying to think. You got me.

Interviewer: I'm just following your lead.

Colton: $\quad$ So, ok, so um. See, I know if you sketch it $\left[\boldsymbol{f}^{\prime}(\boldsymbol{x})\right]$, it will be there, and your derivative is zero, so your graph would have a zero there, but, tangent of a straight line.

Interviewer: What does the tangent, so you just mentioned something about tangents. So why would we even want to look at tangents?

Colton: $\quad$ Because, I mean, our definition of $f$ prime would be the slope of the tangent to any of these points.

Interviewer: Ok. So, if we put a point, like let's say right here [places point on linear portion of graph of $f(x)$ ], what's the tangent line look like there?

Colton: It would be zero [refering to the derivative, not the slope of the tangent line]. Cause the slope is zero. It's not changing.

Interviewer: So, could you sketch it? [the tangent line]

Colton: So, well, I would think. What, a tangent line there?

Interviewer: Uh huh. 
Colton: $\quad$ See, I don't think there would be one because you're on a straight, you're not on a curve. So, I don't know, but then that throws my definition [of the derivative] out too because if you were sketching zero it would go from there to there. So um, I don't know.

Interviewer: So, you're convinced that the derivative exists on this interval and that it's equal to zero?

Colton: Yeah, I'm convinced the derivative there would be equal to zero but then that scratches my definition of a derivative.

Interviewer: But, you're not convinced that the tangent line exists here? But if you say the tangent line doesn't exist here, then you're back to maybe questioning whether or not the derivative does?

Colton: Right.

Interviewer: Is that what you're thinking?

Colton: Well, I mean, I'm pretty certain that this is how you're suppose to sketch the uh [derivative] when you do the curve sketching.

Interviewer: $\mathrm{Ok}$

Colton: $\quad$ So, um I don't know. That's really throwing me there. And then your next question will be on this one too because that will be a straight line so how did I get that too [interval $(e, \infty)]$. Um. Shoot. I don't know.

Interviewer: So, you're saying like if we would put a point here [on $f(x)$ on interval $(e, \infty)$, you're not sure if there's a tangent line at that point?

Colton: $\quad$ Right, because we're not a curve anymore, but then, that ruins my definition of a derivative because I'm basing my definition of a derivative off the tangent of the $y$ function. So, I don't know. Um, I don't know.

His apprehension was due to the requirement for a curve in his concept definition of the tangent line. It was interesting that he said, "I know if you sketch it $\left[f^{\prime}(x)\right]$, it will be there" and "I'm pretty certain that this is how you're supposed to sketch the uh [derivative] when you do the curve sketching." His statements evidenced an internal battle between his concept definition of a tangent line and a process for sketching $f^{\prime}(x)$ that belonged to his concept image of the derivative. Essentially, he knew his sketch of the derivative was correct (and it was), but because his definition of a tangent line required a curve, and because he defined the derivative as being equal to the slope of the tangent line at a point, these two together implied his sketch of the derivative was incorrect. But, he knew it wasn't.

To fix the problem, he considered changing his definition of the derivative so that his sketch of $f^{\prime}(x)$ would remain correct. 
Interviewer: So whenever you were talking about slopes before, you were talking about the shape of the graph and not about slopes of tangent lines?

Colton: Well, I mean, I was thinking about it as change in the slopes as being your derivative. So, when there was no change, I was making it zero, or I was making it, when there was a constant change, I was making it just the value of that ratio. So, but then, I guess that would be ok then, because if you're defining a derivative as instantaneous rates of change, then at these instances, you would have slopes of zero or constant slopes. So, by that definition it would be okay, but by the definition of a derivative being the slope of a tangent line, that makes a caveat. So, it depends, like I said, on the semantics of how you want to make a derivative.

Interviewer: I would like it to be consistent.

Colton: I know. Umm. You're pressing me for a definition. Um, from a physics standpoint, I want to say it's instantaneous rates of change, because, well physics. But, um I think I'm going to go with the derivative being instantaneous rate of change. But then, oh gosh, I don't know. It's killing me between those two. Between how I want to define the definition of the derivative. So, I mean, I want to go with instantaneous rates of change, because that will make my curve sketching valid, I guess.

$\cdots$

Colton: Well, on a curve they're the same [instantaneous rate of change and the slope of a tangent line]. But since we have things where our slope's constant or our slope's zero [linear portions of the graph], that's where I'm running into the issue with that, because then those two don't work [instantaneous rate of change equaling the slope of a tangent line]. Because the slopes of zero, there won't be a tangent on a line that's not a curve. So, that's why I want to run with instantaneous [as my definition of the derivative].That will work, because your slope's zero there and that's zero, but then that, if we do that [define a derivative as instantaneous rate of change], then I'm running into trouble with my definition of a tangent.

Interviewer: So what's causing you trouble is that the tangent line doesn't exist whenever you have a line, like here and like here [intervals $(b, c)$ and $(e, \infty)]$ ?

Colton: Right. So, it all boils down to my definition of a tangent as my problem... If I could, like I said, like go back, if I could just get my definition of a tangent right, I think all this would tie together perfectly. There wouldn't be any conflict. But, I still have my problem with, you can't, I don't think you can take tangents of straight lines.

His concept definition of a tangent line was the root cause of his cognitive conflict. He

reasoned to this conclusion on his own: "it all boils down to my definition of a tangent as my

problem." The interviewer did not probe him in the direction of redeveloping his concept definition of the tangent line, but for time reasons, moved to the next interview task, which was graphically interpreting the formal definition of the derivative. The next section explores the data related to this task. 


\subsubsection{Task 4: Graphically interpreting the formal symbolic definition of the derivative.}

For this task, Colton was asked by the interviewer to graphically interpret the symbols of the formal definition of the derivative. He provided a lengthy response to this question, which essentially consisted of three different attempts. He ultimately was unsuccessful at interpreting the meaning of the symbols, but it was not for a lack of effort. The following presentation of Colton's data is organized in terms of these attempts. A general description of each attempt is discussed first, followed by a table summarizing Colton's many definitions for the involved symbols.

\section{Attempt 1}

In response to being asked to explain the graphical meaning, Colton said, "This is what kills me. I don't know how to say it without just restating the formula." Trying to do something other than just restate the formula, he offered the following:

"You want time to go to zero, because that makes it more accurate. If there's no change in time, you're instantaneous. If your intervals are infinitely smaller, you'll have a more accurate $y$-value when you solve this."

The interviewer followed up with a question regarding what the "more accurate $y$-value" represented. He did not provide an answer, but instead discussed a very procedural interpretation of the definition. He wanted to define $f(x)$. He discussed "plotting many points" to define the function but quickly realized this would be a difficult task; one would have to be "really good at math" or "have a serious computer." Next, he tried to simplify the internal ratio by distributing $f$ to get $\frac{f(x) f(h)-f(x)}{h}=\frac{y f(h)-y}{h}$. He described $f(h)$ as a function of time.

The interviewer attempted to steer him in the direction of a graphical approach, but he did not concede to the prompts. The following are a few examples. First, the interviewer covered up 
the limit notation in the definition of the derivative and asked Colton what the remaining ratio represented graphically. In response, he described $f(x)=y$ as a function in terms of $x$ and $h$ and discussed substituting this into the formal definition and "implicitly differentiating it." Second, the interviewer sketched a general function graph labeled $f(x)$ and placed an $x_{1}$ on the $x$-axis. Colton was asked to graphically interpret $f^{\prime}\left(x_{1}\right)$ using the formal definition of the derivative. He said, "It'd be tricky because you don't have a function." He thought about plugging $x_{1}$ into the derivative function and simplifying the internal ratio to get $\frac{f(x+h)-y}{h}$.

During his explanations, Colton tried twice to recall the expanded version of the denominator as the quantity $(x+h)-x$ in order to assist with his interpretation of the definition of the derivative, but he could not remember "what it would be exactly."

In summary, his first attempt involved trying to use substitution and distribution (both incorrectly) to simplify the difference quotient of the definition. He also referenced implicit differentiation. For his next attempt, the interviewer suggested he refer to his sketch of the derivative from Task 3 and try to make a connection between his definition of the derivative as the slope of the tangent line and the symbols of the formal definition of the derivative.

\section{Attempt 2}

Following the interviewer's prompts, he referred to the graphs from Task 3 and stated, "When you change from $f(x)$ to $f^{\prime}(x)$, your $y$ in $f(x)$ is your location... and your $y$ [in $\left.f^{\prime}(x)\right]$ is your velocity." After this statement, he quit considering the original function graph and worked only with the graph of the derivative. He was using the graph of the derivative function to interpret the symbols in the definition of the derivative. This caused him to get way off track. 
For a point $(x, y)$ of $\boldsymbol{f}$ prime, he considered $\frac{y}{x}$ to be equal to $h$. He thought $\frac{y}{x}=h$ represented the slope of the tangent line to $f^{\prime}(x)$ at $(x, y)$. He wasn't completely satisfied with this solution, correctly so.

He worked insistently, but struggled to figure out the meaning of $h$. In his efforts he said, "I think I'm drawing a circle around something that doesn't exist." The following statement highlights his growing confusion (keep in mind, the mentioned $x$ and $y$ values are on the graph of $\left.\boldsymbol{f}^{\prime}(\boldsymbol{x}), \operatorname{not} f(x)\right)$ :

“Maybe $h$ doesn't equal your tangent then, because slope would be based, slope is a ratio based off of change in $y$ over change in $x$, over change in time. Right? I guess. Right. Cause you'd have. Right. So, slope would be instantaneous. It would be an instantaneous $y$ over $x \ldots$ Slope would be your ratio of $y$ over $x$. Ok. So, that definition works as long as your $h$ is one. Right? Ok, no, because there's no third value there. It would just be $x$ and $y$. But then, we're going for at what rate are those changing? So, you introduce a new variable. So, that would be your $f^{\prime}(x)$ which would be $h$ again $\ldots$ I don't know where $h$ would come into that. I'm trying, I really am."

At this point, it was nearing the end of the allotted time for the interview, and the interviewer offered him the opportunity to stop, but he wanted to "keep at it." This led to Attempt 3 .

Attempt 3

Continuing to work with the graphs of $f(x)$ and $f^{\prime}(x)$ from Task 3 , the interviewer suggested that Colton sketch the tangent line on the graph of $f(x)$ for a specific $x$ value. He did so correctly. Referring to this point of tangency he said, "So the change in this $x$ and this $y$ is the slope of this [pointing to $\left.f^{\prime}(x)\right]$." The interviewer questioned how he was determining "change" 
since he had only one $x$-value and one $y$-value. Unconvinced that it mattered, he said "I don't know" and again considered $\frac{y}{x}$ for the corresponding $(x, y)$ on the graph of the derivative.

He was then asked about the meaning of the limit in the formal definition of the derivative. He said, "I want $h$ to be the change in $x$ and change in $y$. But to do that, we'd have to have some kind of ratio, but the ratio of those two is a slope. So then, you can't do that, because then that screws up this definition [pointing to formal definition for $f^{\prime}(x)$ ]. Because if $h$ is your slope, then that doesn't make any sense. Because $h$ needs to be your change in time."

In a final effort to figure out $h$, he again considered the graphs from Task 3 , and this time, viewed them to represent position and velocity, respectively. He put forth the idea to "redefine" the $y$-axis of $f^{\prime}(x)$ to be "change in $y$," and he called this $h$. This new idea did not lead to anything and he concluded, "I don't know what that'd be. So, I don't know."

Colton's data indicated that even though he thought of the derivative function as representing instantaneous rate of change and the slope of the tangent line, as he described during Task 3 , he did not know how these descriptions of the derivative related to the symbols in the formal definition. He tried diligently to connect the dots, but came up short.

The data showed he did not reference his concept definition of the tangent line during Task 4. This was interesting since he had referenced his concept definition in all previous tasks. The reasoning for this seems to be the nature of his concept definition of the tangent line. It was object-based and did not contain any of the processes of the Tangent Line Framework. The formal symbolic definition of the derivative is given in terms of processes- ratio, limit, and 
function. Therefore, none of these activated elements of Colton's concept definition of the tangent line because these processes do not belong to his personal definition.

\title{
Summary of defined terms
}

During his three attempts, Colton offered various definitions for the terms involved in the

\author{
definition of the derivative. All of these were not discussed in the three attempts above, but are
}

summarized in Table 23. These data accentuate the difficulty Colton had with interpreting the definition of the derivative graphically.

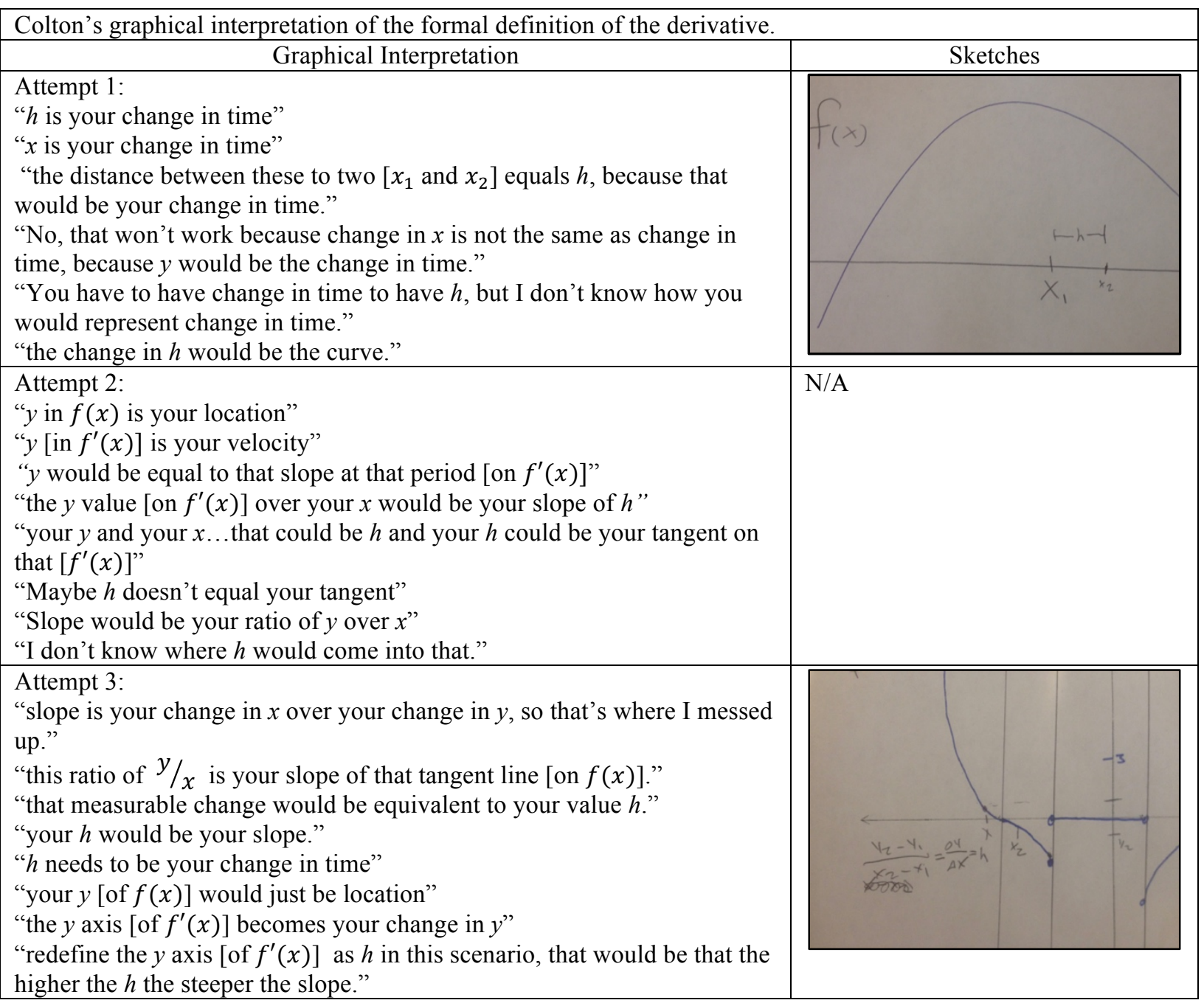

Table 23. Colton. Task 4, Interview 3. Summary chart. 
Colton changed his mind often during Task 4, which made identifying elements of his concept image of the derivative difficult. The elements that were identified are summarized in Table 24, below.

\begin{tabular}{|l|l|}
\hline Colton & \multicolumn{1}{c|}{ Derivative (Tasks 3 and 4) } \\
\hline Elements & $\begin{array}{l}\text { dE1: The slope of the graph of } f(x) \text { determines the value of the derivative function. } \\
\text { When } f(x) \text { has positive slope, the graph of the derivative is above the } x \text {-axis. When } \\
f(x) \text { has negative slope, the graph of the derivative is below the } x \text {-axis. When } f(x) \\
\text { has zero slope, the graph of the derivative is on the } x \text {-axis. When } f(x) \text { has constant } \\
\text { slope, the graph of the derivative is horizontal at a } y \text {-value equivalent to the slope of } \\
\text { the graph of } f(x) . \\
\text { dE2: The graph of the derivative has jump discontinuities at places where the slope } \\
\text { of the graph of } f(x) \text { switches. At these places, the tangent line does not exist and the } \\
\text { graph of the derivative has open circles. } \\
\text { dE2: The derivative of a function is defined as instantaneous rate of change as well }\end{array}$ \\
$\begin{array}{l}\text { as the slope of the tangent line at a point. For a given function graph, these } \\
\text { definitions of the derivative are equivalent when the graph is curved. However, when } \\
\text { the original graph is linear, the tangent line does not exist, so at these places, the } \\
\text { derivative is defined as instantaneous rate of change. } \\
\text { dE4: Infinitely smaller intervals are associated with more accurate "y-values." } \\
\text { dE5: Uncertain of the graphical meaning for the symbols in the definition of the } \\
\text { derivative. }\end{array}$ \\
\hline $\begin{array}{l}\text { CE1: The derivative is equal to the slope of the tangent line at a point. } \\
\text { Element }\end{array}$
\end{tabular}

Table 24. Colton. Tasks 3 and 4, Interview 3. Concept image of the derivative. 


\subsection{Jamie}

\subsubsection{Task 1: Concept definition of the tangent line}

Jamie's concept definitions of the tangent line over the series of interviews are reviewed in the next table, Table 25 . Notice, Jamie's concept definition emphasized the one-point property of tangency associated with its use in geometry. Also, her definition remained fairly consistent over the course of the semester.

\begin{tabular}{|l|l|}
\hline Jamie & Concept definition of the tangent line. \\
\hline Interview 1 & Where it hits one point on a graph. \\
\hline Interview 2 & Where the line intersects at one particular point. \\
\hline Interview 3 & A line that hits at like one point. \\
\hline
\end{tabular}

Table 25. Jamie. Task 1. Summary chart.

It is interesting to point out that even though she defined the tangent line in terms of one point, she did not apply this definition when sketching tangent lines. In a sketching situation, she was ok with the tangent line having multiple or even an infinite number of points in common with the curve. The interviewer addressed this inconsistency throughout the interview. In response, Jamie was typically indifferent towards the value of her definition. The following are a few examples.

During Interview 1, Jamie correctly sketched the tangent line for a given point on a linear function. The interviewer pointed out that the tangent line she constructed had infinitely many points in common with the function, which contradicted her "one-point" definition of the tangent line. The interviewer applied Jamie's definition and suggested a vertical line as the correct tangent line to the linear function. Despite it satisfying her definition, Jamie said that would be incorrect since it looked "kinda weird." Her response evidenced she reasoned based on her intuition rather than the definition of the concept. In other words, her reasoning flowed through her concept image and not her concept definition. 
The questions posed by the interviewer made Jamie aware that her tangent line constructions went against how she defined the tangent line. She realized her definition was insufficient, and said, "I don't know the real definition [of tangent]." She shrugged it off, unconvinced that an accurate concept definition mattered.

As another example, at a corner point on a graph, Jamie correctly said the tangent line would not exist (Task 2, Interview 2). However, based on Jamie's concept definition, one could construct infinitely many "tangent lines" at the given point. The interview asked Jamie about this discrepancy.

Interviewer: If you're going off the "one point" idea, like 'I just need to hit it at one point, then we could draw it like that [sketches incorrect tangent line].

Jamie: $\quad$ Or, you could draw it like that [provides another example of an incorrect tangent line].

Interviewer: $\quad$... So, how do you decide which one it is?

Jamie: The guessing game.

These instances in Jamie's data revealed that her concept definition was inadequate for helping her make decisions about the correct slope of the tangent line. Even though she knew her definition was insufficient, she did not seek to reconstruct her concept definition. It is interesting that in terms of her definition, what Jamie said, was not necessarily what she meant. She defined the tangent line in terms of a one-point property, but then constructed tangent lines with multiple points in common with the graph.

In terms of the Tangent Line Framework, her concept definition aligned with the framework only in terms of the object of the second layer (the limit layer). "One-point" may be thought of as the resulting object of the limit process; the second point on the curve which determines the secant line that approaches the common point. However, Jamie accepted the object without considering the process. This is what Zandieh (2000) calls a pseudo-object. 
Jamie's concept definition of the tangent line is an example of a pseudostructural understanding of tangency.

\subsubsection{Task 2: Construction of tangent lines- six graphs}

This section explores data related to Task 2 of Interview 3, which consists of sketching tangent lines on six graphs. In reviewing the data related to this task, constructing tangent lines, keep in mind Jamie's concept definition of the tangent line and consider the ramifications of this concept definition on her concept image. As shown in Table 26, Jamie constructed the correct tangent line for Graphs 1, 2, and 6, but incorrectly "guessed" the tangent line for the remaining graphs.

\begin{tabular}{|l|l|}
\hline Jamie's tangent line constructions. \\
\hline Graph 1 & "For this one, I'm putting it at the bottom because it is at the bottom of the line [graph]." \\
\hline Graph 2 & "And same [as Graph 1] with this one, cause it's like the same one just flipped." \\
\hline Graph 3 & $\begin{array}{l}\text { "This is how we did it in class. I remember. So, don't ask me why, because I don't know. } \\
\text { I'm just guessing." }\end{array}$ \\
\hline Graph 4 & \begin{tabular}{l} 
"I'm just guessing." \\
\hline Graph 5
\end{tabular} \begin{tabular}{l} 
"And then, with this one, I'm just getting a vibe. It's going to be there [horizontal]." \\
\hline Graph 6
\end{tabular} $\begin{array}{l}\text { "For this one, I feel like it's going to be this way, because the line right here [pointing to } \\
\text { graph near point of tangency]. I don't know, that's just how I feel." } \\
\text { "That's how we did it in class." }\end{array}$ \\
\hline
\end{tabular}

Table 26. Jamie. Task 2, Interview 3. Summary chart.

In her responses, notice that Jamie consulted her concept image and not her concept definition. She recalled processes for sketching the tangent, such as "putting it at the bottom" when the point of tangency was at a local minimum. She also referenced mental pictures of her 
concept image, recalling how she thought the tangent line was sketched "in class." Jamie's explanations show a lack of understanding or appreciation for the mathematical meaning of the tangent line. The understandings she demonstrated during the task do not overlap with the framework. She did not mention any of the process-object pairs of the layers.

To further explore Jamie's elements of Jamie's concept image, the interviewer asked Jamie to provide a bit more detail concerning what she was thinking when constructing her tangent lines

$\begin{array}{ll}\text { Interviewer: } & \text { Whenever you think about how to draw the tangent line to a curve, what } \\ \text { exactly are you trying to do? } & \text { Where it's the best position for it. } \\ \text { Jamie: } & \text { That's what you're trying to decide? } \\ \text { Interviewer: } & \text { Yeah. } \\ \text { Jamie } & \text { And then, how do you know what's the best position for it? What's that } \\ \text { Interviewer } & \text { mean? } \\ \text { Depending on where it is on the graph. So like, for this one [Graph 1], if } \\ \text { the [point of tangency] was here, the line would be this way. Whereas for } \\ \text { this one, it would be on this side. }\end{array}$

Jamie reasoned mainly in terms of the location of the point of tangency. She also referenced her memories from class, which was common for Jamie. Essentially, her process for constructing the tangent line was to consider whether the function was increasing or decreasing at the point of tangency, and then make the tangent line on the "outside" of the graph at the point. 
The location of the point of tangency played a key role in how Jamie thought about the tangent line at a point. As another example, consider her response to the interviewer's question about the slope of the tangent line.

$\begin{array}{ll}\text { Interviewer: } & \begin{array}{l}\text { So, you think of a line that hits at one point [referencing concept definition]. } \\ \text { What about the slope of it? }\end{array} \\ \text { Jamie: } & \text { Um. It is a slope. } \\ \text { Interviewer: } & \text { Ok. } \\ \text { Jamie: } & \text { I don't know. Well, like, do you mean like. } \\ \text { Interviewer: } & \begin{array}{l}\text { What makes it different from any other line? } \\ \text { Jamie: }\end{array} \\ \begin{array}{l}\text { Oh! Depending on where, I have to use my fingers [drawing a curve on the } \\ \text { table with her finger]. Depending on where the tangent line is going to be. } \\ \text { So, if it's like going to be at the top of something, then it will be straight, but if } \\ \text { it's like in the middle, it's going to be a curve. So, it just depends where it's at. }\end{array} \\ \begin{array}{ll}\text { Ok. And then once you know where it's going to be at, how do you decide } \\ \text { about the slope of it? }\end{array} \\ \begin{array}{ll}\text { Whether it's increasing or decreasing. }\end{array}\end{array}$

In response to the interviewer's question about slope, the location of the point of tangency was immediately activated in her concept definition. The preferable response would be for her to consult the limit process that defines the tangent line, like we saw with Aaron. However, Jamie demonstrated a lack of knowledge or lack of appreciation for the limit process. Since Jamie thought about the tangent line as always being on the "outside" of the graph, she considered her tangent line for Graph 6 to be an "exception to the rule," since it cut through the curve at the point of tangency.

Based on Jamie's data from Tasks 1 and 2, elements of her concept image of the tangent line were identified. These elements summarize the evoked portions of her concept image during the interview tasks. Considering these elements in conjunction with her concept definition, an expanded concept definition was derived. The expanded definition encapsulates the meaning of tangency that Jamie conveyed. Jamie's concept definition and expanded concept definition of 
the tangent line as well as identified elements of her concept image are summarized in Table 27 on the next page.

\begin{tabular}{|c|c|}
\hline Jamie & Tangent Lines (Task 1 and 2) \\
\hline $\begin{array}{l}\text { Concept } \\
\text { Definition }\end{array}$ & "A line that hits at like one point." \\
\hline $\begin{array}{l}\text { Elements of } \\
\text { Concept Image }\end{array}$ & $\begin{array}{l}\text { tE1: The tangent line may intersect the function more than once. } \\
\text { tE2: The location of the point of tangency and the shape of the graph determine the } \\
\text { correct tangent line. } \\
\text { tE3: My personal taste, examples from class, and guessing are also used to } \\
\text { determine the correct tangent line. } \\
\text { tE4: The tangent line can cut through the graph at the point of tangency. This is } \\
\text { considered an exception to the rule. }\end{array}$ \\
\hline $\begin{array}{l}\text { Expanded } \\
\text { Concept } \\
\text { Definition }\end{array}$ & $\begin{array}{l}\text { A tangent line is a line that touches the graph at one point and has slope based on } \\
\text { what I feel is the best fit to the graph at that point. }\end{array}$ \\
\hline
\end{tabular}

Table 27. Jamie. Tasks 1 and 2, Interview 3. Concept definition and image of tangent line.

In conclusion, Jamie's data related to Task 2 of Interview 3 revealed that Jamie constructed tangent lines in a way that conflicted with how she defined the tangent line. This did not seem to bother her, and she did not reconstruct her concept definition. Her concept definition of the tangent line was object-focused and did not contain the processes of the Tangent Line Framework. Likewise, her concept image of the tangent line did not contain or prioritize these processes.

\subsubsection{Tasks 3: Sketching the graph of $f^{\prime}(x)$, given the graph of $f(x)$.}

The next task Jamie completed was sketching the graph of the derivative function for a given function graph, for which the formula was not provided. Table 28 on the next page summarizes her work for this task. The quotes are representative of her justifications for each portion of the graph that she was sketching. As a reminder, the red font in the figures was not present during the interview, but was added later for ease of analysis and presentation. 


\begin{tabular}{|c|c|c|}
\hline Jamie & $\begin{array}{l}\text { Original } \\
\text { function graph }\end{array}$ & $\begin{array}{l}\text { Jamie's sketcl } \\
\text { of derivative }\end{array}$ \\
\hline $\begin{array}{l}\text { "Well, I know that from here to here it's a slope of zero. So, because } \\
\text { it's a slope of zero, I can't remember if it's opened or closed, but it's } \\
\text { going to be a line on here [the } x \text {-axis of } f \text { ']." } \\
\text { "Because of these pointy things." }\end{array}$ & & \\
\hline $\begin{array}{l}\text { "So, for this one }[\text { local min], it would still be a slope of zero cause this } \\
\text { is at the bottom, so that's a zero slope, so there's going to be a point } \\
\text { here." }\end{array}$ & & \\
\hline "And this one, I think this one is going to be open too." & & \\
\hline $\begin{array}{l}\text { "This is decreasing, and so I think it would still be like that." } \\
\text { Note: When she said, "this is decreasing", she was referring to the } \\
\text { graph of } f \text { on }(-\infty, b] \text {. }\end{array}$ & & \\
\hline $\begin{array}{l}\text { Made derivative negative where } f \text { was negative, and positive where } f \\
\text { was positive. }\end{array}$ & & \\
\hline $\begin{array}{l}\text { "I can't remember if like it's supposed to be, because like it's } \\
\text { decreasing, but it's like on the positive side and the negative side. So, } \\
\text { I'm confused." } \\
\text { "Well, I know like, it's gonna be something. Something's happening } \\
\text { between here } c c \text { and } d] \text {. I just can't remember if it's like increasing or } \\
\text { decreasing. And, I feel like it would be decreasing [the derivative] } \\
\text { since it's going down [the original function]. }\end{array}$ & & \\
\hline $\begin{array}{l}\text { "Oh that's a slope, too! I forgot about that one!" } \\
\text { (Changes her mind) } \\
\text { "So, that's a point [closed circle at } a \text { on the derivative graph]. So, that } \\
\text { is on the positive side [derivative] since } f \text { is positive [on }[a, b]] . "\end{array}$ & & \\
\hline $\begin{array}{l}\text { "And so then, this is decreasing [reading graph from right to left on (- } \\
\infty, a \text { ], and so I think it would still be like that. Wouldn't it? But, I } \\
\text { know this is right [pointing to the value of zero] for the slope. I know } \\
\text { that is, but this part I can't tell you if I'm right." }\end{array}$ & & \\
\hline
\end{tabular}

Table 28. Jamie. Task 3, Interview 3. Summary chart. 
There were many interesting aspects of Jamie's response to this task. Primarily, what the task revealed concerning how she related the derivative and the tangent line. Jamie placed open circles on the derivative graph where the original function was "pointy." She also placed points on the "derivative line" ( $x$-axis) where the graph of the original function had horizontal tangents. However, Jamie did not know why she was going through these motions.

Open circles:

Interviewer: So, why is that when it's like that [pointy] you draw it open? You're right that you do that, but why?

Jamie: Um, I'm not exactly sure. I can't remember. I remember we were told in class, but I can't remember.

Horizontal tangent lines:

Interviewer: So, how do you know that's a point? Why'd you just go down there and put that point [value of zero on the graph of $\left.f^{\prime}(x)\right]$ ?

Jamie: $\quad$ Because it has a slope of zero.

Interviewer: And why are you looking at slopes?

Jamie: $\quad$ Because that's how we were taught in class.

The interviewer asked Jamie what the tangent line would look like on the graph of $f(x)$ at the places where the graph was "pointy," where she had given the graph of the derivative “open circles" (Figure 11a). In response, Jamie sketched a "tangent line" (Figure 11b). Sketching a tangent line at this point demonstrated a lack of understanding concerning how the tangent line and derivative are mathematically connected. 


\begin{tabular}{|l|l|}
\hline a) Derivative sketch & b) Tangent line to original function \\
\hline &
\end{tabular}

Figure 11. Jamie. Derivative does not exist, but tangent line does.

Jamie's concept definition of the tangent line allowed for the tangent line to exist where the derivative did not. Interestingly, she did not see anything wrong with this discrepancy. Her concept definition of the tangent line put her at a disadvantage for being able to make meaningful connections to the derivative of a function.

When sketching $f^{\prime}(x)$, Jamie considered the slope of the original graph, but only at places where that slope was zero.

Interviewer: So, you're looking at slopes there (local minimum). Are you looking at slopes anywhere else? Or just at zeroes?

Jamie: Just at zeroes.

Interviewer: And then on the other parts that aren't zeroes, like right here, what do you think? Jamie: $\quad Y o u$ determine whether it [the graph of the derivative] goes like down, or it goes up, or if it's like this [an arch].

Interviewer: And then how do you determine that?

Jamie: $\quad$...I feel like if it [the original function] stays above...the zero line and it's positive, that it's going to stay above down here [in the derivative]. Whereas... this one, it's negative so it's going to be negative. I don't know.

Jamie used the horizontal tangent lines of the original function to partition the $x$-axis for the derivative graph. For these intervals, she tried to make the derivative graph correspond with the original graph in terms of positive and negative values. Obviously, she had difficulty making this happen. For example, on one interval, where $f(x)$ had both positive and negative values, she did not know what to do about the derivative function, and stated, "I can't remember if it's supposed to be, because like it's [the original graph] decreasing, but it's like on the positive side 
and the negative side. So, I'm confused." The interviewer suggested she consider a specific $x$ value and reason in terms of a relationship between $f$ and $f$ prime. Then, based on this, make interpretations about the value of the derivative. To get her started, the interviewer marked an $x$ on the $x$-axis of the original graph. The transcript excerpt below shows how Jamie responded to this prompt.

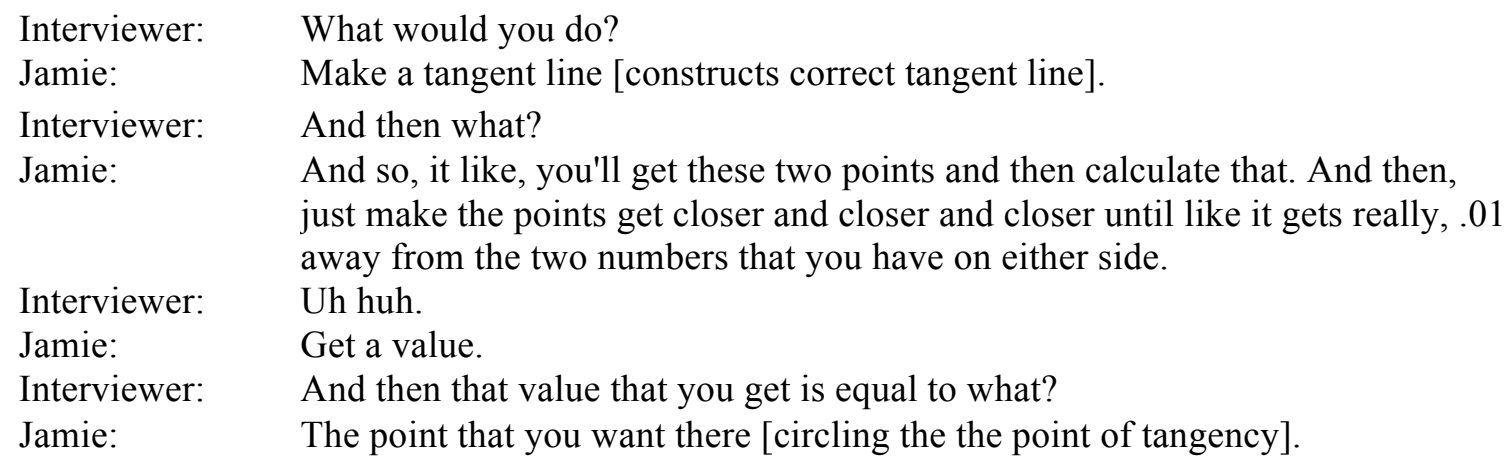

It is unclear what Jamie meant when she said "get these two points and then calculate that." Best case scenario, she was thinking of average rate of change, but there is no evidence of this in the data. She also discussed the points getting "closer and closer and closer," which is language associated with taking a limit. However, she was unable to relate these words to their graphical meaning. She may have been mimicking language from class without an understanding of what the words meant. She eventually concluded that she had "no clue" how the derivative and tangent line were connected.

In conclusion, Jamie related the phrases "tangent line" and "derivative," but never did say the derivative was equal to the slope of the tangent line. Her understandings concerning the exact relationship for these concepts were mixed. For example, she used horizontal tangent lines to assign the derivative values of zero, but also constructed tangent lines to the original graph at places where she said the derivative did not exist. Also, she correctly sketched a tangent line when asked to approximate the derivative, but then said the tangent line approximated the point 
of tangency, and then, never connected it to the derivative. Examining these behaviors in light of her definition of the tangent line, we see that Jamie's definition did not contain the limit process which would have enabled her to connect the concepts in a meaningful way. To this extent, her concept definition of the tangent line contributed to her confusions concerning the derivative.

\subsubsection{Task 4: Graphically interpreting the formal symbolic definition of the derivative.}

When given the symbolic formal definition of the derivative, Jamie said when she sees the symbols, she thinks about how she should "solve it." She proceeded by defining a function, $f(x)=2 x^{2}+x+1$, and plugging it into the definition. She explained, "Whenever there's an $x$, you put an $x+h$." Her ultimate goal was to "simplify it to where I don't have an $h$ on the bottom anymore."

The interviewer asked Jamie about the meaning of $h$ in the definition. She said, "I'm not exactly sure what $h$ is... Should I know what $h$ means?" She guessed, "The $h$ might be a slope of zero." She then sketched a curve and two tangent lines to the curve, one at a local minimum and the other at a local maximum (Table 29). This interpretation of $h$ paralleled her thinking during Task 3, where she considered tangent lines only at places on the graph that were local minimums or maximums.

The interviewer asked Jamie why she was thinking of tangent lines and how they related to the formal definition of the derivative. Jamie responded, "Maybe if you want to find like one exact point on it." The interview ended similar to how it had begun, with a focus on one point. Jamie was not able to reason about the meaning of the derivative beyond how she defined the tangent line. She was not able to evoke a limit or ratio process in her description of the derivative. She was also unable to describe the relationship between the derivative and tangent line, and ultimately concluded, "Maybe there is [a relationship]. Maybe there isn't." 
Jamie mainly viewed the definition of the derivative as something to plug a function into and solve for by canceling $h$ from the denominator. She understood that the resulting function was the derivative function, however, she did not understand the graphical meaning of the involved symbols. Her understanding of the formal definition of the derivative was limited by the pseudo object elements of her definition of the tangent line. Table 29 below summarizes Jamie's Task 4 data.

\begin{tabular}{|l|}
\hline \multicolumn{1}{|c|}{ Jamie's graphical interpretation of the formal definition of the derivative. } \\
\hline "I'm not exactly sure what $h$ is... Should I know \\
what $h$ means?" \\
"The $h$ might be a slope of zero." \\
"Maybe there is [a relationship to tangent lines]. \\
Maybe there isn't."
\end{tabular}

Table 29. Jamie. Task 4, Interview 3. Summary chart.

In conclusion, the elements of Jamie's concept image of the derivative identified from her Tasks 3 and 4 data are outlined in the next table (Table 30). Since Jamie reasoned inconsistently about the relationship between the tangent lines and the derivative, no connecting elements were identified in her data.

\begin{tabular}{|l|l|}
\hline Jamie & \multicolumn{1}{|c|}{ Derivative (Tasks 3 and 4) } \\
\hline Elements & $\begin{array}{l}\mathrm{dE} 1 . \text { There is an open circle on the } x \text {-axis of } f^{\prime}(x) \text { at places where } f(x) \text { is "pointy". } \\
\mathrm{dE} 2\end{array}$ There is a point on the $x$-axis of $f^{\prime}(x)$ at places where the slope of $f(x)$ is zero. \\
$\begin{array}{l}\text { negative. } \\
\text { nes: The definition of the derivative is used to compute } f^{\prime}(x) \text {, given } f(x) . \\
\underline{\mathrm{dE} 5} \text { : The graphical meaning of } h \text { in the definition of the derivative is a slope of zero. }\end{array}$ \\
\hline
\end{tabular}

Table 30. Jamie. Tasks 3 and 4, Interview 3. Concept image of the derivative. 


\subsection{Alice}

\subsubsection{Task 1: Concept definition of the tangent line}

Alice's concept definition across the series of interviews is documented in the next table (Table 31). In reviewing the table, keep in mind that Interview 1 took place the same day that Alice learned the definition of a tangent line, whereas Interview 3 took place at the end of the semester.

\begin{tabular}{|l|l|}
\hline Alice & Concept definition of the tangent line \\
\hline Interview 1 & $\begin{array}{l}\text { It's like a secant line, except on a graph it touches one point. It's a line more so for the } \\
d \text { of } y \text { over } d \text { of } x \text { instead of the change in } y \text { over the change in } x \text {. It's also, I guess, used } \\
\text { for the derivative. It's for the instantaneous rate of velocity, not the average rate of } \\
\text { velocity. }\end{array}$ \\
\hline Interview 2 & $\begin{array}{l}\text { Alice: The tangent line is, I can't remember. I don't know. } \\
\text { Interviewer: What comes to mind? } \\
\text { Alice: At peaks or dips in the graph it would be like a horizontal tangent line. It's } \\
\text { where it's only touching the graph at one point. That's really all that comes to mind. }\end{array}$ \\
\hline Interview 3 & $\begin{array}{l}\text { A tangent line is the slope of a line. I know it has to do with the derivative of a } \\
\text { function. Yeah, that's all I got. }\end{array}$ \\
\hline
\end{tabular}

Table 31. Alice. Task 1. Summary chart.

During Interview 1, it was evident that Alice had just learned a lot of facts related to the tangent line and was still forming her concept definition. She had not yet settled on her own personal meaning of tangent. Her definition resembled a list contrasting a tangent line from a secant line and their associated meanings. During Interview 2, she defined the tangent line as being horizontal "at peaks or dips in the graph." This horizontal aspect of her concept definition took on a more prominent role by the third interview. To demonstrate, consider the following quotes from her third interview:

"[When] I think about drawing tangent lines, I think about where the slope equals zero."

“...all tangent lines are at a point where the slope equals zero."

"Since you're taking the limit as $h$ goes to zero, you're taking it as the slope goes to zero, which is the definition of a tangent line." 
Her descriptions indicate the belief that all tangent lines have a slope of zero, and this will be further examined in the next section.

During Interview 3, she defined the tangent line to be "the slope of a line" that has to "do with the derivative." Based on her definition, one would think she connected the slope of the tangent line to the value of the derivative, however, throughout the entirety of her third interview, she never defined the derivative this way. In fact, she said she was unsure of how the tangent line related to the derivative. Perhaps, the word 'derivative' was present in her concept definition, rather than the concept of derivative.

The next section explores elements of Alice's concept image of a tangent line that were identified during the second interview task- constructing tangent lines to function graphs. Her explanations during the task paint a clearer picture of how she thinks about tangent lines and were used in conjunction with her Task 1 data to derive an expanded version of her concept definition of a tangent line.

\subsubsection{Task 2: Construction of tangent lines- six graphs}

For this task, Alice was presented with six graphs and was asked to sketch the tangent line, if it existed, for each of the given points. Table 32 on the next page summarizes her tangent line constructions and justification for the graphs. 


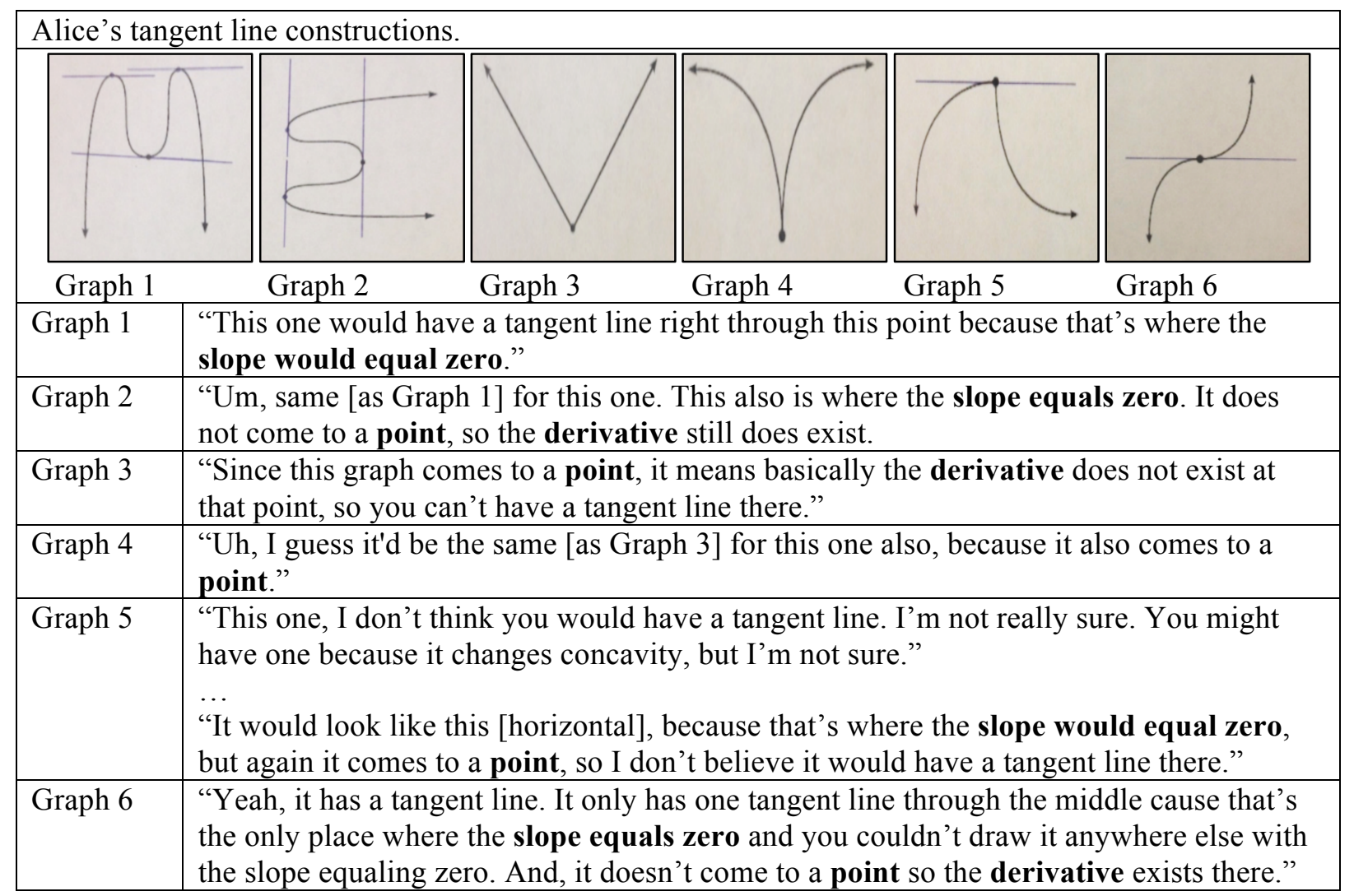

Table 32. Alice. Task 2, Interview 3. Summary chart.

Alice's descriptions primarily emphasized the notions of zero slope, the graph coming to a point, and the derivative not existing. Two of these- slope and derivative- were also referenced in her concept definition of the tangent line. However, some discrepancies exist. For instance, she defined the tangent line as 'slope' during Task 1, but during Task 2, described the tangent line in terms of 'zero slope'. This section explores the 'slope' and 'derivative' elements of Alice's concept image of the tangent line, and examines the meaning she associated with the terms.

Firstly, what does Alice believe about the slope of a tangent line? In the last section, Alice's horizontal tangent line perspective was explored. Her Task 2 data further supports this aspect of her concept image. For instance, consider her justification for her horizontal tangent line in Graph 6: "that's the only place where the slope equals zero and you couldn't draw it 
anywhere else with the slope equaling zero." This indicated the potential belief that tangent lines only exist when the slope of the graph is equal to zero. To explore the details of this potential element of her concept image, the interviewer referred Alice back to Graph 1 and asked about the total number of possible tangent lines for the graph. In response, Alice sketched three tangent lines, each with slope equaling zero. She described these as being the only tangent lines that existed for the graph.

Secondly, what does Alice believe concerning the relationship between tangent lines and the derivative? Looking at her concept definitions of the tangent line from the series of interviews, she referenced the derivative twice:

Interview 1: "It's also, I guess, used for the derivative."

Interview 3: "I know it has to do with the derivative of a function."

Thus, she does connect the phrases 'the derivative' and 'tangent line.' However, from her concept definition alone, the details of this connection are unclear. Her Task 2 data assisted in understanding how Alice connects these two concepts. Alice's justifications for Graphs 2 and 3 shown in the above table, indicate her belief that the shape of the graph determines the existence of the derivative, and in turn, the existence of the derivative determines the existence of the tangent line. For both graphs, her reasoning started with the existence of the derivative and followed to a conclusion about the tangent line. It is important to point out that she never reasoned in the opposite direction, using the tangent line to make interpretations about the derivative.

When asked to explain why the derivative wouldn't exist at places where the graph came to a point, she said, "I'm not really sure. I don't remember." Although she referenced the derivative in her concept definition and used the existence or non-existence of the derivative to 
justify her tangent line sketches, she could not unpack these statements and describe a relationship for the concepts.

For Graph 5, Alice originally stated, “I don't think you would have a tangent line," but then went on to sketch a horizontal tangent line at the given point. She had difficulty making up her mind and was a bit confused, recall:

"It would look like this [sketches horizontal tangent line] because that's where the slope would equal zero, but again it comes to a point, so I don't believe it would have a tangent line there."

Since the graph came to a point, she thought the tangent line would not exist, but since the graph approached a slope of zero from one side, she thought the tangent line should exist and have a slope of zero. These two elements of her concept image were evoked simultaneously and caused her cognitive conflict. Ultimately she did not resolve the conflict, and remained uncertain about what should happen at the point.

In analyzing Alice's data for Task 2, elements of her concept image of the tangent line were identified. These elements together with her concept definition from Task 1, describe how Alice thinks about tangent lines. The identified elements of her concept image provided details to help clarify the vague wording of her concept definition. These elements were used to develop an expanded version of her concept definition. Table 33 on the next page shows her concept definition as well as identified elements of her concept image. An expanded version of Alice's concept definition based on these elements is also displayed in the table. 


\begin{tabular}{|l|l|}
\hline Alice & \multicolumn{1}{c|}{ Tangent Lines (Tasks 1 and 2) } \\
\hline $\begin{array}{l}\text { Concept } \\
\text { Definition }\end{array}$ & $\begin{array}{l}\text { "A tangent line is the slope of a line. I know it has to do with the derivative of a } \\
\text { function. Yeah, that's all I got." }\end{array}$ \\
\hline Elements & $\begin{array}{l}\text { tE1: Tangent lines have a slope of zero. } \\
\text { tE2: Tangent lines do not exist when the graph comes to a 'point,' because the } \\
\text { derivative does not exist at these places. }\end{array}$ \\
\hline $\begin{array}{l}\text { Expanded Concept } \\
\text { Definition }\end{array}$ & $\begin{array}{l}\text { A tangent line is a line with slope always equal to zero. It is related to the } \\
\text { derivative in that if the derivative doesn't exist, then neither does the tangent line. }\end{array}$ \\
\hline
\end{tabular}

Table 33. Alice. Tasks 1 and 2, Interview 3. Concept definition and image of tangent line.

All in all, Alice thought of the tangent line as always having slope equal to zero. Her verbal descriptions of the tangent line indicated that she connected the words 'tangent' and 'derivative,' however, she could not describe a mathematical relationship between the concepts.. Her understandings of the tangent line were limited to the objects of the Tangent Line Framework. During Tasks 1 and 2, Alice made no reference to a ratio or limit process. In this way, her concept definition and concept image of the tangent line were similar in structure.

\subsubsection{Tasks 3: Sketching the graph of $f^{\prime}(x)$, given the graph of $f(x)$.}

This section reviews Alice's data for sketching the graph of the derivative function. In reviewing her data, notice that the element of her concept image concerning the non-existence of the derivative at a corner point was not activated in the context of this task. The next table (Table 34) summarizes Alice's Task 3 data. Be aware, often, what she said did not match what she did. 


\begin{tabular}{|c|c|c|}
\hline Alice & $\begin{array}{l}\text { Original function } \\
\text { graph }\end{array}$ & $\begin{array}{l}\text { Alice's sketch of } \\
\text { derivative }\end{array}$ \\
\hline $\begin{array}{l}\text { Interval }(-\infty, c) \text { : } \\
\text { "The slope is positive and it gets less positive as it } \\
\text { gets to this uh peak right here }(x=a) \text {. Cause when } \\
\text { it gets right to the peak it equals zero. And then from } \\
\text { there, it gets negative- gets less negative and then } \\
\text { gets more negative. The slope is constant there } \\
\text { (interval }(b, c)) \text {. I don't really know. This was my } \\
\text { worst part in calc. Uh, I don't really know. I think it's } \\
\text { just constant in the derivative also, so I'm just going } \\
\text { to draw a line there, I guess." }\end{array}$ & & \\
\hline $\begin{array}{l}\text { Interval }[c, \infty) \text { : } \\
\text { "From this point to this point (interval }(c, d)) \text {, it's } \\
\text { decreasing and the slope is negative, but it gets less } \\
\text { negative as it comes towards where the slope equals } \\
\text { zero. So, I'm going to make it go this way. From here } \\
\text { to here (interval }(d, e) \text { ), the slope starts at zero. It's } \\
\text { less positive and gets more positive as it approaches } \\
\text { the axis here. Um, and then, this is just a positive } \\
\text { constant slope (interval }(e, \infty)) \text {, so I'll do that again } \\
\text { cause it's constant." }\end{array}$ & & e \\
\hline
\end{tabular}

Table 34. Alice. Task 3, Interview 3. Summary chart.

Alice's descriptions seemed to indicate that she was making decisions about the graph of the derivative based on the slope of the graph of the original function. However, what she drew was not consistent with what she was saying. For example, consider interval $(c, d)$ from the above table. She described the slope of the original function as being negative on the interval, but she assigned the derivative both negative and positive values on this interval. For $x=d$, she correctly recognized the slope of the original function to be zero, but then gave the derivative a positive value. She made similar errors for $x=a$ as well as interval $(b, c)$.

The data indicated that she was using the slope of $f(x)$ to determine increasing, decreasing, or constant behavior for the graph of the derivative, but not positive, negative, or zero values. Because Alice did not give the derivative a value of zero at the appropriate places, the interviewer asked her if "there was anywhere the graph of $f^{\prime}(x)$ would be zero?" Alice 
responded, "I'm not sure. Maybe where there is a constant slope." It is intriguing that her concept image of a tangent line was dominated by a horizontal tangent perspective, but then when sketching the graph of the derivative, she neglected to consider horizontal tangent lines in order to determine zeros of the derivative function.

For Tasks 1 and 2, where the context was tangent lines, Alice referenced the derivative. However, during Task 3, where the context was the derivative, Alice did not reference tangent lines. Questions were posed by the interviewer to explore if Alice considered tangent lines when sketching the graph of the derivative, for example:

Interviewer: $\quad$ Talk to me a little bit about why you're looking at slopes.

Alice: $\quad$ Not really sure. Um, because the slope of $f(x)$ will determine if it's increasing or decreasing in $f^{\prime}(x)$, which is not what I drew obviously.

Interviewer: Say that sentence again.

Alice: $\quad$ Um, I think the slope of the line, the slope of the graph in $f(x)$ will determine if $f^{\prime}(x)$ is increasing or decreasing. I think. I'm not sure.

Interviewer: $\quad$ Ok. And then, how do you figure out the slope of the graph of $f(x)$ ?

Alice: $\quad$ Uh, I don't know. I just like look at it and determine if it's going this way, it's negative and if it's going this way, its positive. That's really all I do. [gesturing with her arm a line with negative slope and then a line with positive slope]

Interviewer: Do you think tangent lines play any role, and if so, what?

Alice: $\quad$ I don't think so. [shakes head 'No']

Alice said she used the slope of the original function to determine if the graph of the derivative would be increasing or decreasing. She stated "that's really all I do," which revealed the lack of depth of her understanding. A bit later in the interview, she said, "It's definitely something I really need to study for the final [sketching the derivative], because I just don't remember it and don't understand it, still."

In summary, Alice did not think about tangent lines when determining the graph of the derivative. She considered the slope of the graph of the original function to determine increasing, decreasing, or constant slope in the derivative, and did so correctly. However, she did not know how to determine zero values of the derivative function. This was interesting considering the 
prominent role of horizontal tangent lines in her concept image. She also did not take into consideration places where $f$ was not differentiable. Which was also interesting considering her numerous references to this during Task 2 . In any case, this knowledge was not evoked in the context of Task 3.

\subsubsection{Task 4: Graphically interpreting the formal symbolic definition of the derivative.}

For Task 4, Alice was asked to graphically interpret the symbols of the formal definition of the derivative. This task activated elements of Alice's understanding that were not evidenced in any prior tasks. Upon seeing the symbols of the definition, she said:

"If you were to take the limit away, I would think of this as the change in $y$ over the change in $x$, which would just be the slope of a secant line of the function, and then, when you take the limit as $h$ goes to zero, it uh, then it becomes the tangent line."

Alice demonstrated knowledge of the ratio process when she said, "change in $y$ over change in $x$." She also mentioned secant lines, which was new vocabulary for her third interview. She went on to say that "it becomes the tangent line." However, she did not demonstrate an understanding of how these words related to the limit process is the definition. When asked to elaborate on the limiting process, she explained:

"Since you're taking the limit as $h$ goes to zero, you're taking it as the slope goes to zero, which is the definition of a tangent line. It's where the slope equals zero on the derivative of a function."

She related the limit towards zero with slopes approaching zero, and then immediately connected this to "the definition of a tangent line," saying it is where "the slope equals zero." This was a valuable piece of data in that it was consistent with her expanded concept definition of the tangent line from Tasks 1 and 2, which emphasized a horizontal perspective of tangency. 
Also note, Alice referenced "slope," "tangent line," and "derivative" in the above quote, but on the previous task, she never used the slope of the tangent line to reason about the derivative.

Following her explanation, she was asked to provide a sketch. She very hesitantly put together the sketch shown in the next table (Table 35). Despite her correct sketch, she evidenced much uncertainty, and ultimately shied away, stating, "I can't. I'm sorry." As such, her sketch most likely represented a memorized figure from class stored in Alice's concept image.

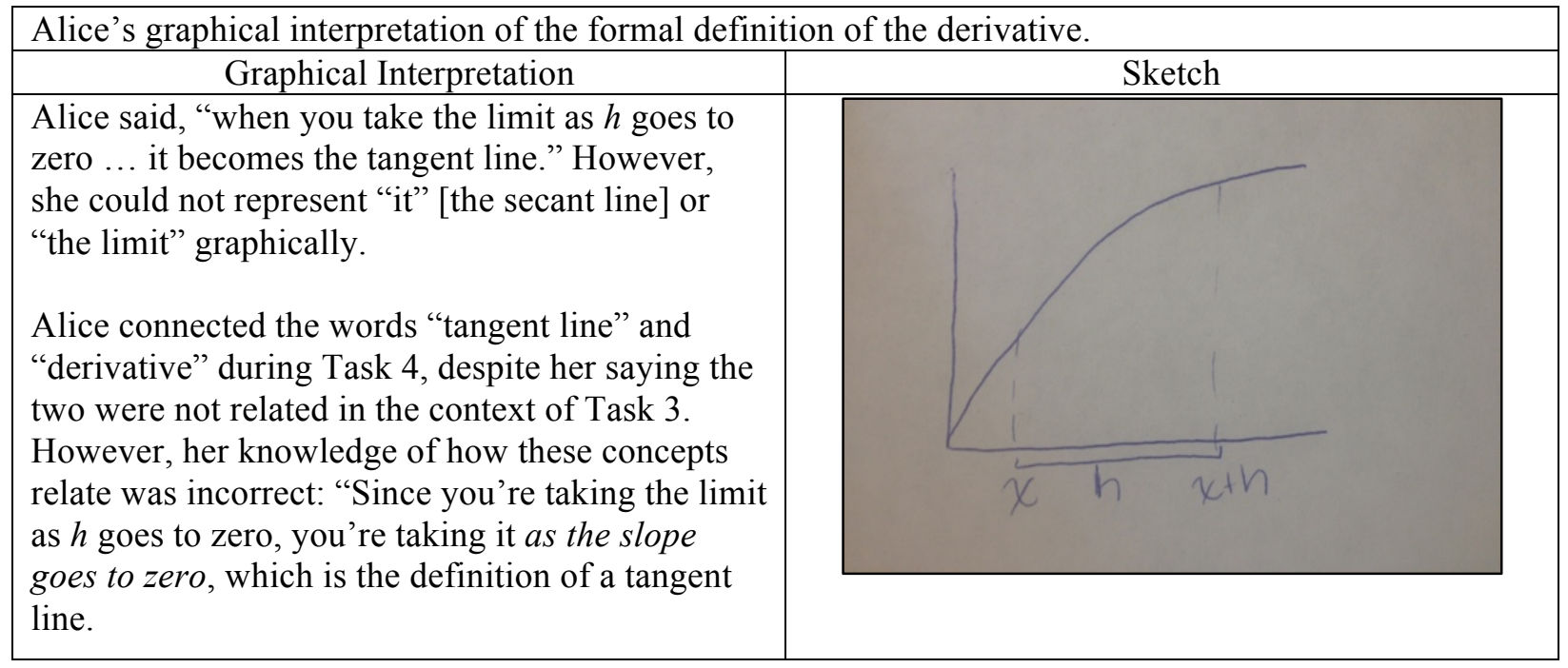

Table 35. Alice. Task 4, Interview 3. Summary chart.

In summary, Alice was not able to accurately describe the limit process of the definition of the derivative. Comparatively, her concept definition of the tangent line also did not include the limit process. Likewise, for both the derivative and the tangent line, Alice emphasized slopes of zero. These data indicate ways Alice's understandings for the tangent line and derivative overlapped.

The identified elements of Alice's concept image of the derivative are reviewed in the next table (Table 36). Although Alice occasionally associated the words tangent line and derivative, she never equated the slope of the tangent line at a point to the value of the derivative 
function at that $x$. Due to her inconsistencies, no connecting elements were identified in her concept image.

\begin{tabular}{|l|l|}
\hline Alice & \multicolumn{1}{|c|}{ Derivative (Tasks 3 and 4) } \\
\hline $\begin{array}{l}\text { Concept Image } \\
\text { of the }\end{array}$ & $\begin{array}{l}\text { dE1: The slope of the graph of } f(x) \text { determines if the graph of } f^{\prime}(x) \text { is increasing, } \\
\text { decreasing, or constant. } \\
\text { dE2: Tangent lines do not play a role in determining the graph of the derivative, } \\
f^{\prime}(x), \text { for a given function graph, } f(x) . \\
\text { dE3: In the definition of the derivative, } \frac{f(x+h)-f(x)}{h} \text { represents change in } y \text { over } \\
\text { change in } x, \text { which is the slope of a secant line. Taking the limit as } h \text { goes, results in } \\
\text { the tangent line. } \\
\text { dE4: Tangent lines are related to the definition of the derivative because as } h \text { goes to } \\
\text { zero, the slopes are going to zero, and the definition of the tangent line is where the } \\
\text { derivative has a slope of zero. }\end{array}$ \\
\hline $\begin{array}{l}\text { Connecting } \\
\text { Element }\end{array}$ & \begin{tabular}{l} 
N/A \\
\hline
\end{tabular}
\end{tabular}

Table 36. Alice. Tasks 3 and 4, Interview 3. Concept image of the derivative. 


\subsection{Tangent Line Framework}

The concept definitions of the tangent line for the twelve participants of this study are reviewed in this section. Table 37 shows these definitions for the four core students, and Table 38 shows these data for the remaining eight participants. Student references to the process-object pairs labeled: L1O, L1P, L2O, and L2P denote the objects and processes for layers one and two, respectively. In reviewing the tables, notice students' tendencies towards defining the tangent line in terms of pseudo-objects. 


\begin{tabular}{|c|c|}
\hline \multicolumn{2}{|r|}{ Concept definition of the tangent line over the series of interviews. } \\
\hline \multicolumn{2}{|r|}{ Aaron } \\
\hline Int. 1 & $\begin{array}{l}\text { A tangent line is a line that intersects a function... well, it touches, just briefly touches it at one } \\
\text { point (L2O) and um it's involved where you find the, you differentiate a function you find the } \\
\text { derivative of it, it's used in that. }\end{array}$ \\
\hline Int. 2 & $\begin{array}{l}\text { A tangent line would be a representation of the slope (L1O) at a certain point. It would just } \\
\text { follow um... you pick a certain point }(\mathbf{L 2 O}) \text {, like there, and then you would trace a line, and } \\
\text { that would represent the slope (L1O) narrowing it down to that point }(\mathbf{L 2 O}) \text { right there. }\end{array}$ \\
\hline Int. 3 & $\begin{array}{l}\text { It is the line that represents the, it has a slope (L1O) of a function at a certain point (L2O). As } \\
\text { opposed to a secant line (L1O), where it's like connecting two points on a function and is the } \\
\text { line that goes in between them. As the second point approaches the first point, it's like the } \\
\text { limit of that (L2P). And that's the tangent line (L2O). It represents the slope. }\end{array}$ \\
\hline \multicolumn{2}{|r|}{ Colton } \\
\hline Int. 1 & The slope (L1O) of a curve at a single point (L2O) \\
\hline Int. 2 & The line that touches a curve at one point (L2O) and has its slope (L1O) at that point. \\
\hline Int. 3 & $\begin{array}{l}\text { A line or a plane that touches a curve at one spot (L2O) and if you continue, won't touch it } \\
\text { again. }\end{array}$ \\
\hline \multicolumn{2}{|r|}{ Alice } \\
\hline Int. 1 & $\begin{array}{l}\text { It's like a secant line (L1O), except on a graph it touches one point (L2O). It's a line more so } \\
\text { for the } d \text { of } y \text { over } d \text { of } x \text { instead of the change in } y \text { over the change in } x \text {. It's also, I guess, used } \\
\text { for the derivative. It's for the instantaneous rate of velocity, not the average rate of velocity. }\end{array}$ \\
\hline Int. 2 & $\begin{array}{l}\text { At peaks or dips in the graph it would be like a horizontal tangent line. It's where it's only } \\
\text { touching the graph at one point (L2O). }\end{array}$ \\
\hline Int. 3 & A tangent line is the slope (L1O) of a line. I know it has to do with the derivative of a function. \\
\hline \multicolumn{2}{|r|}{ Jamie } \\
\hline Int. 1 & Where it hits one point (L2O) on a graph. \\
\hline Int. 2 & $\begin{array}{l}\text { Where the line intersects at one particular point (L2O), but it can hit other points, but you } \\
\text { just want to find the number point. }\end{array}$ \\
\hline Int. 3 & A line that hits at like one point (L2O). \\
\hline
\end{tabular}

Table 37. Tangent Line Framework. Four core students. 
Concept definition of the tangent line over the series of interviews.

Jacob

1 A line that connects to one point (L2O) along a graph, and it can intersect the graph.

2 A line that represents the slope (L1O) at a particular point (L2O)

3 A line that represents the slope (L1O) of the graph at a particular point (L2O).

\section{Corey}

1 First thing I think of is back in geometry when you have a circle and the tangent line intersects the circle at only one point (L2O), but now, being in calculus, I know that it's more than just that. It still just intersects one point on a curve, but it is the instantaneous slope (L1O), or rate of change, at that point.

2 The slope (L1O) at that point. The equation of a line showing a slope at a certain point, intersecting the original function in one specific place (L2O).

3 It is a line that will show you the instantaneous rate of change at a point (L2O) on a graph.

Anita

1 The tangent line was like, we had a graph drawn, and you were manipulating it on the computer, and it was the line that was just kinda like going along the outside of the graph.

2 It only intersects one point (L2O), I think. I'm always confused about that, because I feel like there are exceptions, so I'm not sure. It's more so just like finding the slope (L1O).

3 It's the slope of the secant line (L1O). It only touches the graph at one point (L2O). I'm not sure about the formal, formal definition, but I know that's what it is.

\section{Mindy}

1 I thought it was like only the trig function, but I realized, it's like a line that touches one point (L2O) only on a function.

2 The slope (L1O) of the function, I think, at a given point (L2O).

3 The slope (L1O) of a point, like one point (L2O).

Kevin

1 A line that skims the function, so at the curve of the function it skims it.

2 It's the opposite of a secant line. It kind of skims the function, barely touches it. It's where it's right at the curve.

3 It skims the function. It can pass through, but it usually doesn't. The closer the tangent line and the uh, I'm drawing a blank, the other line, the closer they are together, the more they are, they're going to line up with the function itself.

\section{Amanda}

1 A line that only touches a curve at one point (L2O).

2 It only hits the graph at one point (L2O), but it can cross through other parts of the graph, but it's only at like a certain point.

3 A line that passes through any point of the curve but it has to at that specific time (L2O).

\section{Kenneth}

1 A line that intersects a graph or another line, whether it be continuous or not, at the point, at one point (L2O) only.

2 The line that's tangent to it when you draw it, it's supposed to be as close to the curve as possible when it reaches the point.

3 The line parallel to the point that's given.

$$
\text { Carly }
$$

1 There's a point on the line, and it's a line that touches the point on a function.

2 Where the point is on the graph, and then the line would be showing the slope (L1O) at that point (L2O). It's just a straight line.

3 The tangent line is the same as the derivative at a point (L2O) on a function.

Table 38. Tangent Line Framework. Remaining eight participants. 


\section{Chapter 6 - Results}

\subsection{Introduction}

In this chapter, the understandings evidenced by the students are defined in terms of overlap or lack of overlap with the Tangent Line and Derivative Frameworks. These frameworks describe the formal definition of the concepts in terms of layers of process-object pairs and were outlined in Chapter 3 of this dissertation. To this extent, these are used to illustrate the aspects of the formal definition to which each student had accessed. Additionally, the frameworks serve as tools for comparing these aspects across concepts, for the tangent line and the derivative. As such, the results concerning how these understandings relate are discussed.

The previous chapter provided an in depth analysis of four of the twelve participants in the research study. In this chapter, I will compare that analysis to the other eight students in the study and discuss major themes related to the students' concept definitions and images of the tangent line and the derivative.

The results of data analysis presented in this chapter are sub-divided into six sections, the first two of which pertain to the research questions that guided this dissertation study:

Research Question 1: What are first-semester calculus students' concept definitions and concept images of the tangent line? How do these relate to one another and compare to the formal definition of the tangent line at the first-semester calculus level?

Research Question 2: How do first semester calculus students connect the concepts of the tangent line and the derivative in a graphical representation?

Following the results for each research question, this chapter concludes with a discussion of general themes, implications for teaching, limitations of the study, and future research 
projects. Throughout this chapter, assume the existence of two different "cells" in a student's cognitive structure- one for the concept definition and the other for the concept image. 


\subsection{Research Question 1: What are first-semester calculus students' concept definitions and concept images of the tangent line? How do these relate to one another and compare to the formal definition of the tangent line at the first-semester calculus level?}

\subsubsection{Task 1}

This section discusses the analysis of the twelve students on the first interview question of the series, which asked them to define the tangent line. Each student's response was considered his or her concept definition of the tangent line. These definitions are listed in Tables 37 and 38 of Chapter 5.

The next table, Table 39, demonstrates which aspects of the formal definition were emphasized by the students in their definitions of the tangent line for the series of interview. The number in each cell represents how many students (out of twelve) referenced that aspect of the formal definition.

\begin{tabular}{|l|c|c|c|c|}
\hline \multirow{2}{*}{$\begin{array}{c}\text { Overlap with the } \\
\text { framework }\end{array}$} & \multicolumn{2}{|c|}{ Layer One (Ratio) } & \multicolumn{2}{c|}{ Layer Two (Limit) } \\
\cline { 2 - 5 } & Process: Ratio & Object: Slope & Process: Limit & Object: One Point \\
\hline Interview 1 & 0 & 3 & 0 & 9 \\
\hline Interview 2 & 0 & 8 & 0 & 10 \\
\hline Interview 3 & 0 & 4 & 1 & 8 \\
\hline
\end{tabular}

Table 39. All twelve participants' concept definitions. Alignment with Tangent Line Framework.

The overwhelming majority of students defined the tangent line by emphasizing the "objects" of the layers, either slope or one point, and only one student defined the tangent line in terms of a limit process (Aaron, Interview 3).

Examining the last column of the table, we see that students remained fairly consistent in their references to a one-point property with the curve. However, examining the object column for layer one, we do not see this same trend, but rather, a reference to slope occurred twice as often during the second interview. This may due to the timing of Interview 2, which took place as participants were learning applications of tangent lines. These applications often emphasize 
slope and therefore, this aspect of the definition was more prominent in their thinking and thus, more accessible to them during Interview 2.

Another interesting result from the table is that overlap with the framework did not increase as the series of interviews progressed. This is surprising, since educators may often expect a student's concept definition to increase in alignment with the formal definition over the course of a semester, as he or she develops a deeper understanding of calculus. However, the students of this study did not demonstrate such a tendency; their definitions did not increase in sophistication over the course of the semester, in terms of overlap with the framework.

In terms of Interview 1, which occurred shortly after students' first encounters with the formal definition, a reference to the ratio or limit process was still lacking. None of the twelve students mentioned these processes in their personal definitions of the concept. This result points to a hesitancy on the part of students to accept these processes as defining features of the tangent line. It also demonstrates students' casual approach to the meaning of tangency in calculus, and their tendency to describe the concept in terms of pseudo-objects. This may be due to students' difficulties with the notion of limit in calculus (Tall, 1992), or may be attributed to their difficulties with the very nature of mathematical definitions (Edwards \& Ward, 2004).

The mathematical community's notion of understanding the concept of the tangent line and the way first-semester calculus students define the concept, differ most greatly in that students' typically neglect the processes inherent in the formal definition. At this point, one may wonder if these processes, although absent from a student's concept definition of the tangent line, may belong to his or her concept image. The next section explores results related to this possibility. 


\subsubsection{Task 2}

Task 1 data represents the students' concept definitions of the tangent line, whereas Task 2 data helps to explore the students' concept images. This section discusses the results related to how these two sets of data compare. It is often the case that a student's concept image is a much larger structure compared to his or her concept definition. For this reason, it is quite possible that even though a student does not define the tangent line using a ratio or limit process, these processes may belong to his or her concept image of the tangent line.

For Task 2, students were provided with six graphs and were asked to construct the tangent line, if it existed, for each indicated point on the graphs. The graphs for which the tangent line existed, the students were asked to justify the correctness of their tangent line sketch and explain their thought processes for sketching the tangent line. In cases of graphs on which the tangent line did not exist, the students were asked to explain why. In order to provide accurate justifications, students would need to access their knowledge of the formal definition. In this way, the sketching tasks served as stimuli for eliciting the ratio and limit processes within a student's concept image, if, in fact, he or she possessed knowledge of these processes. To this extent, students were provided an opportunity to demonstrate a deeper level of understanding than evidenced by their definitions in the previous task.

The results revealed that students' ways of thinking about the tangent line, in large part, paralleled the phrases they used to define the concept. In other words, students' justifications for the slope of a tangent line and their descriptions for how to construct a tangent line, did not add to the understandings evidenced by their definition of a tangent line. The next two tables model the knowledge demonstrated by each student in terms of alignment with the formal definition of the tangent line in calculus. Table 40 represents their concept definitions and Table 41 represents 
their concept images. Recall from previous descriptions, shaded circles denote structural understandings, whereas open circles signal pseudostructural understandings.

\begin{tabular}{|l|c|c|}
\hline & \multicolumn{2}{|c|}{ Tangent Line Framework } \\
Layers: & Layer One & Layer Two \\
\hline Aaron & 0 & 0 \\
\hline Colton & 0 & 0 \\
\hline Alice & 0 & 0 \\
\hline Jamie & & \\
\hline
\end{tabular}

Table 40. Concept definition of the tangent line. Interviews 1-3, Task 1. Four core students.

\begin{tabular}{|l|c|c|}
\hline & \multicolumn{2}{|c|}{ Tangent Line Framework } \\
Layers: & Layer One & Layer Two \\
\hline Aaron & 0 & 0 \\
\hline Colton & 0 & 0 \\
\hline Alice & 0 & 0 \\
\hline Jamie & & \\
\hline
\end{tabular}

Table 41. Concept image of the tangent line. Interview 3, Task 2. Four core students.

The identical models reveal a key result of this study- students' concept definitions of the tangent line indicate the prioritized elements of their concept images. Students who neglected the limit process in their definitions, demonstrated a general lack of knowledge of this process for the tangent line. These students placed higher value on memories from class or personal intuitions than on the formal definition. For example, consider Jamie, who when sketching the tangent line for Graph 3 said, "I'm going to go with this way, because I think this is how we did it in class. I remember. So, don't ask me why, because I don't know. I'm just guessing." The "tangent line" she recalled from class was incorrect. Contrast that with Aaron, who recognized the limit process as a defining feature of the tangent line and used it to justify the correctness of his sketches. For instance, on Graph 6, he said, "The secant line would be like that, and then, you're just sliding this point closer and closer and closer and the limit as this point, like the distance of these two, as that limit approaches zero, as this point gets infinitely closer to that, you end up with a line like that [the tangent line]." 
Results related to Tasks 1 and 2 indicate a close connection between the words or phrases used by a student to define the tangent line and the elements prioritized within their concept images. The direction of influence is uncertain. Are their concept definitions insufficient as a result of underdeveloped concept images or are their concept images weakened by lacking concept definitions? In either case, the results indicate that students tend not to associate the tangent line with a limit process, but instead, reason about the tangent line based on incorrect intuitions. Little, if any, attention is given to developing an accurate concept definition that aligns with the formal definition. In turn, students tend to demonstrate concept-image based ways of reasoning about the tangent line. These are primarily influenced by classroom memories or procedures aimed at constructing a tangent line with one point in common with the graph. 


\subsection{Research Question 2: How do first semester calculus students connect the concepts of the tangent line and the derivative in a graphical representation?}

\subsubsection{Task 3}

For Task 3, the students were given a general function graph, for which the algebraic representation was not provided, and were asked to sketch the derivative. For this type of task, first-semester calculus students are typically taught to estimate the value of the derivative at any value of $x$ by considering the tangent at the point $(x, f(x))$ and estimating its slope. The slope of the tangent line becomes the $y$-value on the graph of $f^{\prime}(x)$. The task draws upon the relationship between the value of the derivative function and the slope of the tangent line, and in this way, was incorporated in the interview protocol to access student knowledge of this connection.

Of the four core participants, and even when considering all twelve, Colton was the only student who provided a sketch of the derivative function that was considered correct, other than the filled in circle at the first jump, which he later corrected. Aaron's sketch was basically correct with two minor errors- the graph approaching negative and positive infinity for the last two discontinuities. On the other hand, Jamie and Alice's sketches were mainly incorrect, but did contain some correct features. Hence, Aaron and Colton performed similarly on the task as did Alice and Jamie. Likewise, the two groups evidenced comparable thought processes- Aaron and Colton consulted a definition for the derivative during the task, whereas Alice and Jamie did not. The next table, Table 42, displays each student's derivative sketch for Task 3. 


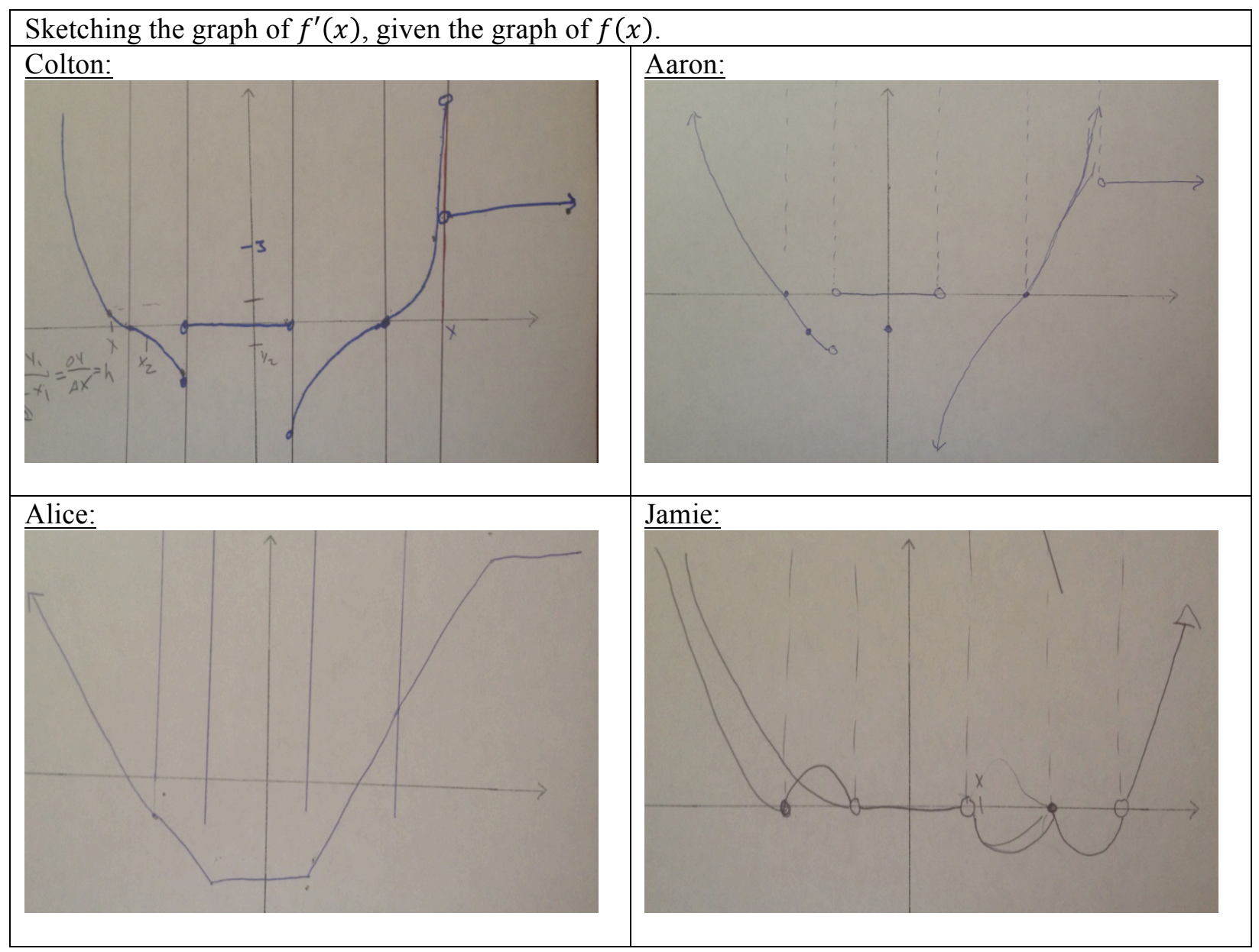

Table 42. Interview 3, Task 3. Summary chart. Four core students.

Jamie and Alice were able to produce graphs of the derivative that had some correct features. Alice's graph increases, decreases, and is constant on the correct intervals, and Jamie's has the correct $x$-intercepts and correctly identified values for which the derivative does not exist. However, these behaviors were characteristic of pseudo-analytical behaviors, which are produced by pseudo-analytical thought processes (Vinner, 1997). These terms identify, respectively, behaviors and processes of thought that take place without understanding. For example, reflecting on her sketch, Alice said, “I personally don't think it's right... I never really understood. It's definitely something I really need to study for the final, because I just don't remember it and don't understand it still." 
On the other hand, Colton and Aaron were able to produce (mainly) correct graphs of the derivative. Both students defined the derivative to be equal to the slope of the tangent line at a point, and both students used this definition to reason about the graph of the derivative. However, Colton experienced cognitive conflict during the task due to his definition of the tangent line, whereas Aaron did not. Colton's definition of the tangent line as not existing for linear functions implied his sketch of the derivative graph was incorrect, but Colton knew his sketch was correct. When his definition of the tangent line was activated in conjunction with his process for sketching the derivative, and these two did not align, he experienced cognitive conflict. He reasoned about this dissonance quite well, stating, "It all boils down to my definition of a tangent as my problem... If I could just get my definition of a tangent right, I think this would all tie together perfectly." Contrastingly, Aaron's definition of the tangent line was compatible with the formal definition and did not cause him any problems. Thus, although both students were successful at sketching the derivative, because of how they defined the tangent line, one experienced cognitive conflict and the other did not. Aaron's definition served as a stepping stone for making meaningful connections to the derivative, whereas Colton's was a hurdle that limited his understanding and caused him cognitive conflict.

The different categories of thought demonstrated by the participants during Task 3 may be best represented by Vinner's models of thinking during problem solving (1991). These depict mental actions of consulting one's concept definition or image when working on a mathematical task (Figure 12). Aaron and Colton are representative of the first thought process (Figure 12a), where the subject consults both his or her concept image and definition, while Jamie and Alice are representative of the second thought process (Figure 12b), where the subject consults only his or her concept image. 


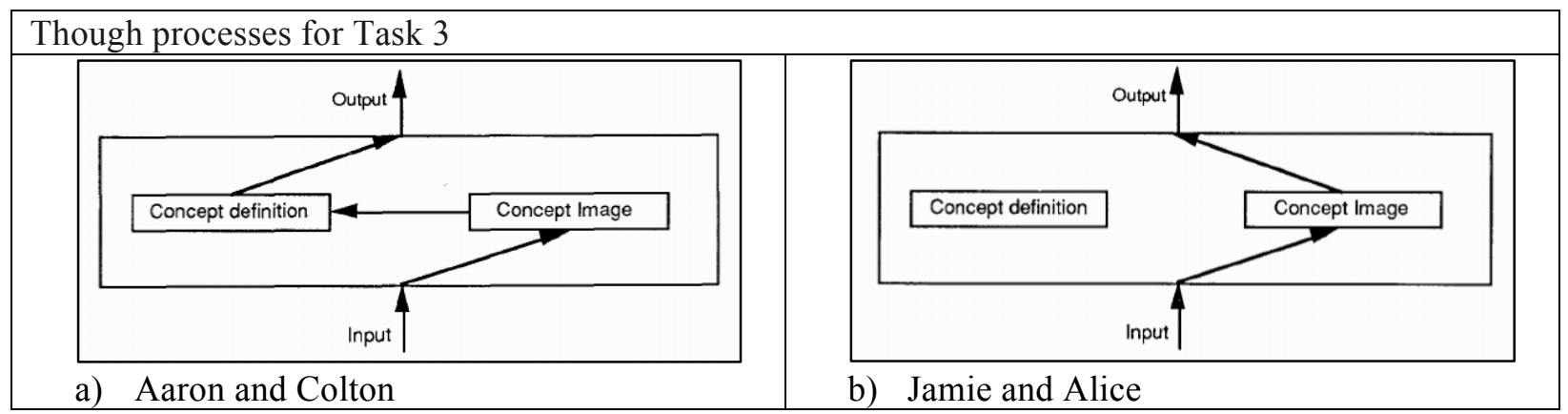

Figure 12. Two different thought processes.

Comparing students' thought processes when sketching the derivative to their definitions of the tangent line, there are interesting results to consider. Alice and Jamie defined the tangent line in terms of pseudo-objects, and then, did not consult a definition for the derivative during the sketching task. In fact, neither student ever stated that the slope of the tangent line is equal to the derivative, and when asked if tangent lines and derivative were related, Alice stated, 'I don't think so," while shaking her head "No," and Jamie said she had "no clue." They demonstrated concept-image based ways of reasoning. They attempted to apply procedures for sketching the derivative to which they associated little meaning. For instance, Jamie assigned the derivative a value of zero when the tangent line to the original graph was horizontal "because that's what we were taught in class." Contrastingly, Aaron defined the tangent line in terms of a limit process, and then, did consult a definition for the derivative during the sketching task. Somewhat in the middle, Colton, like Alice and Jamie, defined the tangent line in terms of pseudo-objects, but, like Aaron, did consult a definition for the derivative during the sketching task. However, unlike Aaron, he experienced cognitive conflict due to his definition of the tangent line. Together, these results suggest that students' abilities to apply definitions or processes for sketching the derivative are influenced by their definitions of the tangent line. Students with more sophisticated definitions, like Aaron, were more likely to demonstrate fluency in recalling and applying necessary information related to the derivative, whereas students with less formal 
definitions of tangency, like Alice, Colton, and Jamie, were less likely to demonstrate this ability and more likely to experience cognitive conflict. These results demonstrate the far reaching implications of students' concept definitions of the tangent line.

Cognitive barriers exist for students' with inadequate definitions of tangency. Most notably, a tendency to not equate the value of the derivative and the slope of the tangent line at a point. The analysis suggests that students' pseudo-object based definitions of the tangent line are the primary factors contributing to these restricted understandings.

\subsubsection{Task 4}

Results of the first research question indicate calculus students' emphasis on pseudoobjects when defining the tangent line, and an overwhelming tendency to neglect the ratio and limit processes of the formal definition. This section considers results related to students' abilities to elicit these processes in a different context, the formal symbolic definition of the

derivative. For Task 4, students were provided the symbols of the definition, $\lim _{h \rightarrow 0} \frac{f(x+h)-f(x)}{h}$, and were asked to discuss their graphical meanings.

Table 43, on the next page, reviews the four cores students' definitions of the tangent line from Interview 3 and excerpts from that interview for their graphical interpretation of the derivative definition. 


\begin{tabular}{|c|c|c|c|}
\hline \multicolumn{4}{|c|}{ Interview 3} \\
\hline \multirow[t]{2}{*}{ Student } & \multirow{2}{*}{$\begin{array}{l}\text { Task 1- Concept } \\
\text { definition of the tangent } \\
\text { line }\end{array}$} & \multicolumn{2}{|c|}{$\begin{array}{l}\text { Task 4- Graphical interpretation of the formal symbolic } \\
\text { definiton of the derivative. }\end{array}$} \\
\hline & & Limit & Difference quotient \\
\hline Aaron & $\begin{array}{l}\text { "It is the line that } \\
\text { represents the, it has a } \\
\text { slope of a function at a } \\
\text { certain point. As opposed } \\
\text { to a secant line, where it's } \\
\text { like connecting two points } \\
\text { on a function and is the } \\
\text { line that goes in between } \\
\text { them. As the second point } \\
\text { approaches the first point, } \\
\text { it's like the limit of that. } \\
\text { And that's the tangent line. } \\
\text { It represents the slope." }\end{array}$ & $\begin{array}{l}\text { "You're closing it in and as that } \\
\text { distance, } h \text {, as you're moving } \\
\text { this point towards the other } \\
\text { point, um the } h \text { grows smaller. } \\
\text { And, as it approaches, the } \\
\text { distance approaches zero. As } \\
\text { you're getting infinitely closer } \\
\text { to that [other point], this } \\
\text { number [circling } h \text { ] keeps } \\
\text { getting smaller and smaller and } \\
\text { towards zero. And then, you } \\
\text { would find, you'd solve that and } \\
\text { then you would find the slope } \\
\text { of the tangent line at that point, } \\
\text { or the derivative." }\end{array}$ & $\begin{array}{l}\text { "If you were to have a } \\
\text { secant line, it would be like, } \\
\text { you have points A, and then } \\
\text { that's coordinates }\left(x_{1}, y_{1}\right) \\
\text { and then another one up } \\
\text { here would be coordinates } \\
\left(x_{2}, y_{2}\right) \text {. And, it's basically } \\
\text { the same concept as this } \\
\text { [pointing to } \frac{f(x+h)-f(x)}{h} \text { of } \\
\text { the definition], [which] } \\
\text { represents the slope." }\end{array}$ \\
\hline Alice & $\begin{array}{l}\text { "A tangent line is the slope } \\
\text { of a line. I know it has to } \\
\text { do with the derivative of a } \\
\text { function. Yeah, that's all I } \\
\text { got." }\end{array}$ & $\begin{array}{l}\text { "Since you're taking the limit } \\
\text { as } h \text { goes to zero, you're taking } \\
\text { it as the slope goes to zero, } \\
\text { which is the definition of a } \\
\text { tangent line. It's where the } \\
\text { slope equals zero on the } \\
\text { derivative of a function." }\end{array}$ & $\begin{array}{l}\text { "I would think of this as the } \\
\text { change in } y \text { over the change } \\
\text { in } x \text {, which would just be } \\
\text { the slope of a secant line of } \\
\text { the function." }\end{array}$ \\
\hline Colton & $\begin{array}{l}\text { "A line or a plane that } \\
\text { touches a curve at one spot } \\
\text { and if you continue, won't } \\
\text { touch it again." }\end{array}$ & "Your $h$ would be your slope." & $\begin{array}{l}\text { "Slope is your change in } x \\
\text { over your change in } y . "\end{array}$ \\
\hline Jamie & $\begin{array}{l}\text { "A line that hits at like one } \\
\text { point." }\end{array}$ & $\begin{array}{l}\text { "I'm not exactly sure what } h \\
\text { is... Should I know what } h \\
\text { means?". }\end{array}$ & $\begin{array}{l}\text { "The } h \text { might be a slope of } \\
\text { zero." }\end{array}$ \\
\hline
\end{tabular}

Table 43. Tasks 1 and 4, Interview 3. Four core students.

Notice that Aaron's rich definition of tangency allowed him to easily relate the symbols

of the derivative to their graphical meaning. Making such a connection was expected of Aaron since his definition of the tangent line involved the limit process. Somewhat unexpected was Colton, Alice, and Jamie's inability to recall these relationships within the given context. Tall and Vinner (1981) point out that interactions with different stimuli evoke different aspects of a student's concept image. For instance, Aaron, Alice, and Colton did not define the tangent line in 
terms of a ratio process, but this process was activated in the context of Task 4 because of the difference quotient stimulus. In a similar way, the limit symbol of the formal definition could have activated the limit process in their concept images, but it did not. These results are concerning since educators may expect students who do not emphasize the limit process when defining the tangent line to be able to elicit this process in other contexts, such as the formal definition of the derivative. However, the students of my study did not demonstrate such an ability. Table 44, below, illustrates how each student's concept definition of the tangent line compares to his or her knowledge of the formal definition of the derivative

\begin{tabular}{|c|c|c|c|c|}
\hline \multicolumn{2}{|c|}{$\begin{array}{l}\text { Coded Charts for Tangent Line and } \\
\text { Derivative Frameworks }\end{array}$} & $\begin{array}{l}\text { Layer One } \\
\text { (Ratio) }\end{array}$ & $\begin{array}{l}\text { Layer Two } \\
\text { (Limit) }\end{array}$ & $\begin{array}{l}\text { Layer Three } \\
\text { (Function) }\end{array}$ \\
\hline \multirow{2}{*}{ Aaron } & Tangent Line & & & --------------------- \\
\hline & Derivative & & & \\
\hline \multirow{2}{*}{ Colton } & Tangent Line & ) & 0 & ----- \\
\hline & Derivative & & D & 0 \\
\hline \multirow{2}{*}{ Alice } & Tangent Line & ) & & ------ \\
\hline & Derivative & & 0 & \\
\hline \multirow{2}{*}{ Jamie } & Tangent Line & & 0 & -------------------- \\
\hline & Derivative & 0 & 0 & \\
\hline
\end{tabular}

Table 44. Tasks 1 and 4. Tangent Line Framework. Four core students.

Aaron was the only student who described the limit process for the Derivative and Tangent Line Frameworks. Recall from the Data Chapter, upon seeing the symbols of the definition in Task 4, he quickly associated these to his concept definition of the tangent line, stating, “...you're just building on concepts.” His structural understanding (shaded circle) of the limit process for the tangent line, transferred to a structural understanding of the limit process for the derivative. A similar transfer of knowledge took place for Colton, Alice, and Jamie; their pseudostructural understandings (open circles) of the limit process for the tangent line, transferred to pseudostructural understandings of the limit process for the derivative. 
In conclusion, students' verbal expressions for connecting the notions of tangent line and derivative are typically not representative of meaningful thought processes. For instance, Alice defined the tangent line as having "to do with the derivative of a function," Colton defined the derivative to be equal to the slope of the tangent line at a point, and Jamie associated zeros of the derivative graph with horizontal tangent lines. Each of these students associated the words tangent line and derivative but were unable to connect the concepts. Graphically contrasting results related to Tasks 1 and 4 (Table 44), a key factor of this conceptual gap is the lack of limit process in students' concept definitions of the tangent line.

\subsection{Conclusions}

\subsubsection{Major themes}

Analysis of the four primary interview tasks revealed interesting results concerning how students think about the tangent line and how these understandings relate to their knowledge of the derivative. The key results related to the four tasks are the following:

Task 1: First-semester calculus students tend to define the tangent line in terms of pseudo-objects, most notably, slope and one-point. As a result, they neglect the processes which formally define the concept.

Task 2: Students' concept definitions of the tangent line indicate the prioritized elements of their concept images of the tangent line.

Task 3: Research based mental blockages associated with the derivative, such as understandings dominated by algebraic techniques, difficulties with visualization, or inability to equate the derivative to the slope of the tangent line at a point (Habre \& Abboud, 2006, Asiala et al., 1997), were not experienced by students with accurate definitions of tangency, whereas such mental blockages were experienced by students 
with less sophisticated tangent line definitions. These results suggest a few possibilities: students' pseudo-object based definitions of the tangent line produce and/or contribute to such mental blockages, students' pseudo-object based definitions are inadequate for overcoming such mental blockages.

Task 4: Overwhelmingly, students who did not define the tangent line in terms of a limit process, demonstrated an inability to evoke this process in the context of the formal symbolic definition of the derivative.

\subsubsection{Discussion}

A student's concept image of the tangent line contains all the mental pictures and processes that he or she associates with that concept. This internal structure is considered to be much larger than a student's corresponding concept definition, which is the form of words used by the student to define the tangent line. Interestingly, my research has shown that the words a student uses to define the tangent line are indicative of the ideas they most strongly associate with that concept. In other words, a student's concept definition represents the prioritized elements of their concept image.

Students who define the tangent line in terms of pseudo-objects, also reason about the tangent line in terms of pseudo-objects. When probed and given the opportunity to demonstrate deeper understandings, these students did not reference the processes which formally define the concept. In this way, their concept definitions and concept images were equivalent in terms of alignment with the framework of this dissertation.

Not to be misunderstood, a pseudo-object is not meant to always carry a negative connotation. It is often simpler (and even necessary) to describe a concept using pseudostructural 
"objects." However, deep understandings are demonstrated by an ability to evoke this information when probed or in mathematically appropriate contexts.

Although it may not be surprising that calculus students' definitions of the tangent line fall short of the formal definition of the concept, it is surprising that their concept images do not pick up the slack, so to speak. The limit process of the formal definition should be one of the top elements activated within a student's concept image when asked to give a rationale for the slope of the tangent line at a point. However, the students of this study who did not define the tangent line in terms of a limit process, also did not elicit this process during other tasks involving tangent lines. These results point to the importance of the words and phrases used by a student to define tangency.

This dissertation study also looked at first-semester calculus students' understanding of the derivative in a graphical representation. Students with a more sophisticated concept definition of the tangent line, such as Aaron, defined the derivative as the slope of the tangent line and used this definition to reason about the derivative. Students with less sophisticated concept definitions, such as Alice, Colton and Jamie, experienced various obstacles as well as cognitive conflict.

In conclusion, the prevailing tendency of first-semester calculus students is to define the tangent line using short-cut definitions, and in turn, allow these notions to dominate the way they think about the tangent line at large. Unfortunately, their concept definitions tend not to evolve over the course of a semester, and as a result, their understandings related to applications of tangent lines appear to be only surface level. 


\subsection{Teaching implications}

At the secondary and collegiate level, many instructors expect a one-way process for the formation of a student's concept image; they expect the concept image to be formed and controlled by the concept definition (Vinner, 1991). In the case that a student's concept definition does not align with the formal definition of that concept, this ideal notion of concept formation is problematic. This dissertation study highlighted this problematic situation in regard to students' concept definitions and images of the tangent line. In light of these results, what instructional practices may facilitate students' development of a structural conception of the tangent line?

Sfard (1991) noted in her work that the "formation of a structural conception is a lengthy, often painfully difficult process" (p. 9). We see this theory playing out in students' conceptions of the tangent line. Aaron and Colton, who had previous calculus experience, demonstrated deeper understandings of the tangent line compared to Alice and Jamie, who were taking calculus for the first time. In fact, a similar trend is present in the data for all twelve participants. Comparing average interview ratings to previous calculus experience, students with previous calculus experience tended to perform better than their peers who were taking calculus for the first time. This data is presented in Table 45 on the next page. The table is organized by average interview ratings- the students in the upper half (blue) received ratings of Excellent or Good and the students in the bottom half (gold) received ratings of Ok or Poor. 


\begin{tabular}{|l|l|l|l|l|l|l|l|l|}
\hline Name & Aaron & Colton & Amanda & \multicolumn{2}{l|}{ Carly } & \multicolumn{2}{l|}{ Corey } \\
\hline Average Rating & Excellent & \multicolumn{2}{|l|}{ Good } & Good & \multicolumn{2}{l|}{ Good } & \multicolumn{2}{l|}{ Good } \\
\hline $\begin{array}{l}\text { Previous Calculus } \\
\text { Experience }\end{array}$ & Yes & \multicolumn{2}{|l|}{ Yes } & \multicolumn{2}{l|}{ Yes } & \multicolumn{2}{l|}{ Yes } & \multicolumn{2}{l|}{ Yes } \\
\hline Name & Alice & Jacob & Mindy & Jamie & Anita & \multicolumn{2}{l|}{ Kenneth } & Kevin \\
\hline Average Rating & Ok & Ok & Ok & Poor & Poor & Poor & Poor \\
\hline $\begin{array}{l}\text { Previous Calculus } \\
\text { Experience }\end{array}$ & No & Yes & No & No & No & Yes & Yes \\
\hline
\end{tabular}

Table 45. Average interview rating compared to previous calculus experience.

The table indicates that students with an average interview rating of Excellent or Good all had previous calculus experience, whereas the majority of students with average interview ratings of Ok or Poor were taking calculus for the first time (four of seven). None of the firsttime calculus students received an average rating of Excellent or Good. Thus, when teaching tangent lines in calculus, it is important to be cognizant of students who are meeting the notion for the first time and try to provide them with multiple opportunities to become acquainted with the formal definition of the concept. One suggestion is to refrain from descriptions that emphasize pseudo-object aspects of the concept, such as "a line that touches the curve at one point," and instead, incorporate process-focused explanations, such as "the tangent line is the limiting position of secant lines." As often as possible, provide visual representations of secant lines approaching the tangent line and encourage students to sketch this process as well. Repeated exposure to the formal definition as well as visual representations can aide students in the process of forming a structural conception of the tangent line.

Interestingly, Aaron was the only student who had taken calculus at both the high school and collegiate level, and was also the only student to demonstrate a concept image and definition of tangency influenced by the formal definition of tangent as a limit process. Because of his limit definition of tangency, Aaron was able to grasp the derivative concept at a deeper level than his peers in the study. These results indicate that an emphasis on limit is imperative for students to 
build an accurate concept definition and image of tangency that will allow them to make meaningful connections to the derivative. However, research has indicated that limit-based concepts, like the tangent line or the derivative, are often developed without a connection to the formal definition of limits (Beynon \& Zollman, 2015). Without understanding the essential components of a formal limit definition, students often struggle to understand limit based mathematical concepts (Roh, 2010). In contrast, students who possess an accurate and workable concept definition of limits are more likely to be successful in solving limit-based calculus problems (Przenioslo, 2004). Research has also indicated that even though instructors are fluent in thinking of limit as a number (or end state) and limit as a process, students tend to only think of limit as a process (Gulcer, 2012). This may be why students of my study tended not to connect the slope of the tangent line (a number or end state) to a limit process. To discourage pseudostructural conceptions of slope related to tangency, educators should emphasize that the slope of the tangent line is a number and that this number is a limit.

The process-object pairs of the Tangent Line Framework refer to inseparable, though dramatically different, facets of the same thing. Calculus instruction should take this duality into consideration and refrain from emphasizing objects (slope and one point) over processes (ratio and limit). Edwards and Ward (2008) noted that although textbooks and many calculus instructors do not emphasize formal definitions, we must begin to consider the implications of these pedagogical decisions on students' mathematical understandings. Instruction should encourage concept-definition based reasoning over concept-image based reasoning.

The students of this study often referenced their instructors' tangent line sketches in order to justify the correctness of their own tangent lines, and in some cases, recalled these tangent lines incorrectly. A recommendation for educators is to encourage students to engage in 
construction tasks, rather than passively watching the instructor. This may help increase students' abilities to recall correct tangent lines. Additionally, students should engage in tasks related to graphically illustrating the limiting position of secant lines. This may help students form an association between the tangent line and the limit process, and assist with moving this process to the foreground of their concept images.

The challenge for educators is to give students experiences that will enable each of them to come to a complete understanding of the process-object pair for each layer of the Tangent Line Framework. Since the processes of the framework are not intuitively prioritized by students, extra care must be taken to encourage appreciation for these processes.

\subsection{Limitations}

A limitation of this dissertation study was the graphically focused context of the interview questions. Given different stimuli, such as tasks involving instantaneous rate of change or velocity, students may have demonstrated more robust understandings. However, Zandieh's study (2000) showed that calculus students prefer graphical contexts (by the end of the semester). In her study, five of the nine students used 'slope' most often to describe the derivative, while the other four students used 'rate' most often, with 'slope' as a close second. To this extent, students who did not elicit the limit process in a graphical representation, may be less likely to do so in other representations of the concept.

Another limitation of the current study was using the frameworks to structure and describe students' understandings of the tangent line and the derivative. The frameworks were valuable tools for analyzing an individual's understanding in terms of the formal definition of that concept and comparing these understandings across concepts, however, the frameworks were limited in that they did not capture students' "incorrect" ways of thinking. 
Other limitations include the involved subjects and interview procedures. For instance, the twelve students of this study may not be representative of all calculus students at the institution in which the study took place, and may certainly not be representative of calculus students at other universities. Also, it is possible that the most dedicated students were the ones who volunteered for the study, and in this case, again, the subjects would not be a representative sample. The interview procedures may also have influenced the students' performances on the interview tasks. The presence of the video camera may have inhibited students' responses. Some students may have felt more comfortable in front of the video camera, and in turn, were able to think clearer and provide responses that were representative of their knowledge on the subject, whereas other students may have experienced anxiety due to the presence of the video camera, and as a result, were unable to elicit necessary information. One way to address this perhaps bias, would be to provide students with the interview questions and allow them time to think about the concepts prior to turning on the video camera. Another option would be to send a follow up email or schedule an appointment for students to view their interviews. These would give students the opportunity to expound on their thought processes and possibly display understandings outside of what they demonstrated in their interviews. Furthermore, while I had very narrow windows for the timing of each interview, it was possible that some subjects received more or less instruction prior to each interview.

\subsection{Future Research}

For future research projects, I would like to conduct a similar study with subjects enrolled in higher level mathematics courses, such as Calculus II or III or Real Analysis. It would be interesting to explore these students' concept definitions and images of the tangent line and see 
what aspects of the framework they emphasize as well as identify other understandings and incorrect ways of thinking outside of the framework.

In this study, I did not collect data concerning classroom instruction. For future research projects, I would like to look at calculus classroom discourse for the topics of the tangent line and the derivative. The frameworks would serve as tools for documenting which aspects of the concepts are involved in student reasoning and teacher questions and explanations. This would involve expanding the Tangent Line Framework to include representations other than graphical, such as verbal, physical, and symbolic. 


\section{REFERENCES}

Amit, M., \& Vinner, S. (1990). Some misconceptions in calculus: Anecdotes of the tip of the iceberg? In George Booker, Paul Cobb, \&T.N. de Mendicuti (Eds.), Proceedings of the $14^{\text {th }}$ International Conference for the Psychology of Mathematics Education, 1, (pp. 3-10). Oaxtepee, Mexico: CINVESTAV.

Asiala M., Cottrill J., Dubinsky, E. \& Schwingendorf, K.E. (1997). The development of students' graphical understanding of the derivative. Journal of Mathematical Behavior, 16:339-431.

Biza, I. (2011) Students' evolving meaning about tangent line with the mediation of a dynamic geometry environment and an instructional example space. Technology, Knowledge and Learning. 16(2), 125-151.

Biza, I., Christou, C., \& Zachariades, T. (2008). Students' perspectives of the relationship between a curve and its tangent in the transition from Euclidean Geometry to Analysis. Research in Mathematics Education, 10 (2), 53-70.

Beynon, K. \& Zollman, A. (2015). Lacking a rigorous concept of limit: Advanced nonmathematics students' personal concept definitions. Investigations in Mathematics Learning, $8(1), 47-62$.

Davis, R., \& Vinner, S. (198). The notion of limit: Some seemingly unavoidable misconception stages. Journal of Mathematical Behavior, 5, 181-203

Edwards, B., (1997). Undergraduate mathematics majors' understanding and use of formal definitions in real analysis, unpublished doctoral dissertation, Pennsylvania State University.

Edwards, B.S., \& Ward, M.B. (2004). Surprises from mathematics education research: Student (mis)use of mathematical definitions. The American Mathematica; Monthly, 111(5), 411-424

Edwards, B.S., \& Ward, M.B. (2008). The role of mathematical definitions in mathematics and in undergraduate mathematics courses. In Making the Connection: Research and Teaching in Undergraduate Mathematics Education (Vol. 73, pp. 223-232.)

Fischbein, E. (1987). Intuition in science and mathematics: An educational approach. Dordrecht, The Netherlands: Reidel.

Goldin, G. (2000). A scientific perspective on structured, task based interviews in mathematics education research. In A. E. Kelly \& R. Lesh (Eds.), Research design in mathematics and science education (pp. 517-545). New Jersey: Lawrence Erlbaum.

Gulcer, B. (2012). Examining the discourse on the limit concept in a beginning-level calculus classroom. Educational Studies in Mathematics, 82 (3), 439-453.

Gravemeijer, K. (1999). How emergent models may foster the constitution of formal mathematics. Mathematics Thinking and Learning, 1, 155-177. 
Habre, S. \& Abboud, M. (2006). Students' conceptual understanding of a function and its derivative in an experimental calculus course. The Journal of Mathematical Behavior, 25(1), 57-72.

Hahkioniemi, M. (2006). Associative and reflective connections between the limit of the difference quotient and limiting process. Mathematical Behavior (25). 170-184.

Harel, G., \& Tall, D.O. (1991). The general, the abstract, and the generic in advanced mathematics. For the Learning of Mathematics, 11(1), 38-42.

Meel, D. (2003). Models and theories of mathematical understanding: Comparing Pirie and Kieren's model of the growth of mathematical understanding and APOS theory. CBMS Issues in Mathematics Education, 12, 132-181.

Orton, A. (1983). Students' understanding of differentiation. Educational Studies in Mathematics, 14, 232-250.

Przenioslo, M. (2004). Images of the limit of function formed in the course of mathematical studies at the university. Educational Studies in Mathematics, 55(1-3), 103-132.

Roh, K.H. (2010). An empirical study of students' understanding of a logical structure in the definition of limit via the $\varepsilon$-strip activity. Educational Studies in Mathematics, 73(3), 263279.

Sfard, A. (1991). On the dual nature of mathematical conceptions: Reflections on processes and objects as different sides of the same coin. Educational Studies in Mathematics, 22, 1-36.

Stewart, J. (2015). Calculus: Early transcendentals ( $8^{\text {th }}$ ed.). Boston, MA, United States: Brooks/Cole, Cengage Learning.

Strauss, A., \& Corbin, J. (1990). Basics of qualitative research: Techniques and procedures for developing grounded theory. Newbury Park, CA: Sage.

Tall, D.O. (1987). Constructing the concept image of a tangent. In J.C. Bergerom, N. Herscovics $\&$ C. Kieran (Eds.), Proceedings of the $11^{\text {th }}$ conference of the international group for the psychology of mathematics education (Vol. 3, pp. 69-75). Montreal, Canada.

Tall, D. (1992). The transition to advanced mathematical thinking: Functions, limits, infinity, and proof. In D.A. Grouws (Ed.), Handbook of Research on Mathematics Teaching and Learning (pp. 49-511). New York: MacMillan Publishing Company.

Tall, D. \& Vinner, S. (1981). Concept image and concept definition in mathematics with particular reference to limits and continuity. Educational Studies in Mathematics, 12, 151169.

Vincent, B., LaRue, R., Sealey, V., \& Engelke, N. (2015). Calculus students' early concept images of tangent lines. International Journal of Mathematical Education in Science and Technology. 46(5), 641-657. 
Vinner, S., \& Dreyfus, T. (1989). Images and definitions for the concept of function, Journal for Research in Mathematics Education, 20, 356-366.

Vinner, S. (1991). The role of definitions in the teaching and learning of mathematics. In D. Tall (Ed.), Advanced Mathematical Thinking (pp. 65-80). Dordrecht: Kluwer Academic Press.

Vinner, S. (1997). The pseudo-conceptual and the pseudo-analytical thought processes in mathematics learning. Educational Studies in Mathematics, 34, 97-129.

Winicki-Landman, G. \& Leikin, R. (2000). On equivalent and non-equivalent definitions: Part 1. For the Learning of Mathematics, 20(1), 17-21.

Zandieh, M. (2000). A theoretical framework for analyzing student understanding of the concept of derivative. In E. Dubinsky, A.H. Schoenfeld, \& J. Kaput (Eds.), Research in Collegiate Mathematics Education IV. (pp. 103-127). Providence, RI: American Mathematical Society.

Zandieh, M. \& Rasmussen, C. (2010). Defining as a mathematical activity: A framework for characterizing progress from informal to more formal was of reasoning. The Journal of Mathematical Behavior, 29(2), 57-75.

Zimmerman, W. (1991). Visual thinking in mathematics. In W. Zimmerman \& S. Cunningham (Eds.), Visualization in teaching and learning mathematics (pp. 127-137). Washington, DC: Mathematical Association of America. 


\section{APPENDIX A: Questionnaire}

Name

e-mail

phone number

May I send you a text message reminder for each of your interviews? yes or no (circle one)

1. Compared to other subjects, my confidence level in math is (Circle one)

$\begin{array}{cccc}1 & 2 & 3 & 4 \\ (\text { low }) & & & \text { (high) }\end{array}$

2. Have you learned about tangent lines before?

3. How would you define a tangent line?

4. Sketch two to three examples of tangent lines. 
5. Sketch the tangent line at the following points, if it exists.

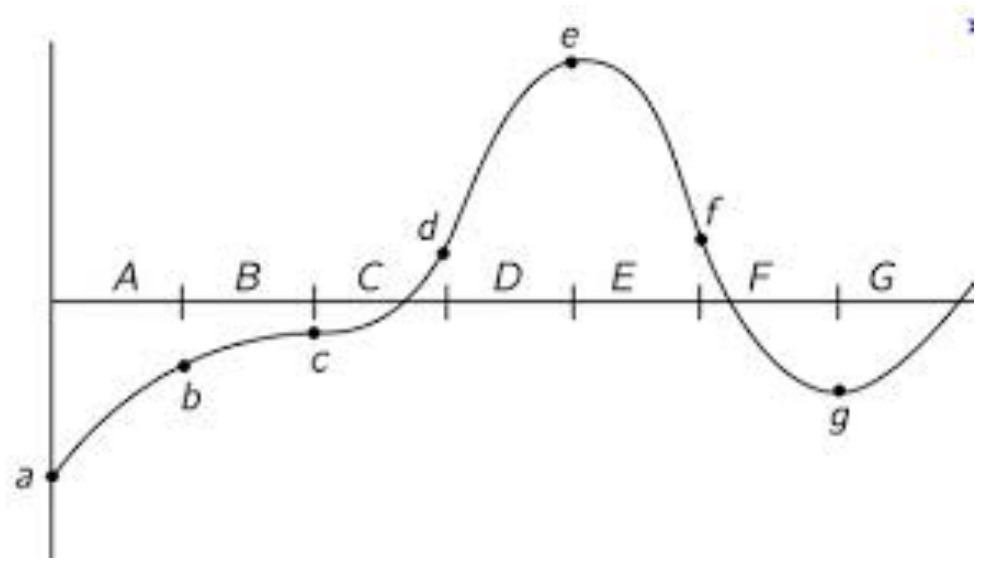

6. When sketching a tangent line to a curve, what are you thinking?

7. In your previous math courses, how were you taught to sketch tangent lines? Do you still think about that process when constructing tangent lines?

8. Have you seen tangent lines in geometry as a tangent to a circle?

9. How does the tangent to a circle in geometry relate to tangent lines used in calculus (if you've had calculus)? 
8. What is your current status at WVU (Circle one)?

Freshman Sophomore Junior Senior Graduate Student Other (please specify)

9. What is your major? (List your desired major if it has not yet been declared.)

10. How long has it been since you took (and passed with a $\mathrm{C}$ or higher) a math course (Circle one)?

Less than one year ago

$1-2$ years ago

$2-5$ years ago

more than 5 years ago

11. Have you previously taken a calculus course (Circle one)? This includes high school/college, passed/failed, or courses dropped.

Yes, I have previously taken a calculus course No, I am taking calculus for the first time.

12. If your answer to question 11 was "No", you may skip to question 13. If you have previously taken calculus, please answer the following series of questions.

a) Have you taken calculus at WVU in a previous semester? Circle all that apply.

MATH 150: Applied Calculus

MATH 153: Calculus 1a with Precalculus

MATH 154: Calculus 1b with Precalculus MATH 155: Calculus 1 (Non-Engineering)

MATH 155: Calculus 1 (Engineering)

b) Have you taken calculus at another college Circle all that apply?

I took calculus at another college and received transfer credit

I took calculus at another college but did not receive transfer credit.

c) Did you take calculus in high school? Circle all that apply.

AP Calculus I took another calculus class in high school (not AP)

13. Participating in this research project requires you to complete a series of three interviews throughout the semester. It is important that you are committed to participating in each interview. You will be paid $\$ 10$ per interview plus a bonus for completing all interviews. Below are the expected dates. Please review the following dates, and then, circle ANY interview that you will NOT be able to attend.

INTERVIEW 1: February 10, 11, or 12

INTERVIEW 2: March 18, 19 or 20

INTERVIEW 3: April 28, 29, or 30 
APPENDIX B: Interview 1

Name

1.) What is the definition of a tangent line?

2.) Can you provide an example?

3.) Constructing tangent lines:
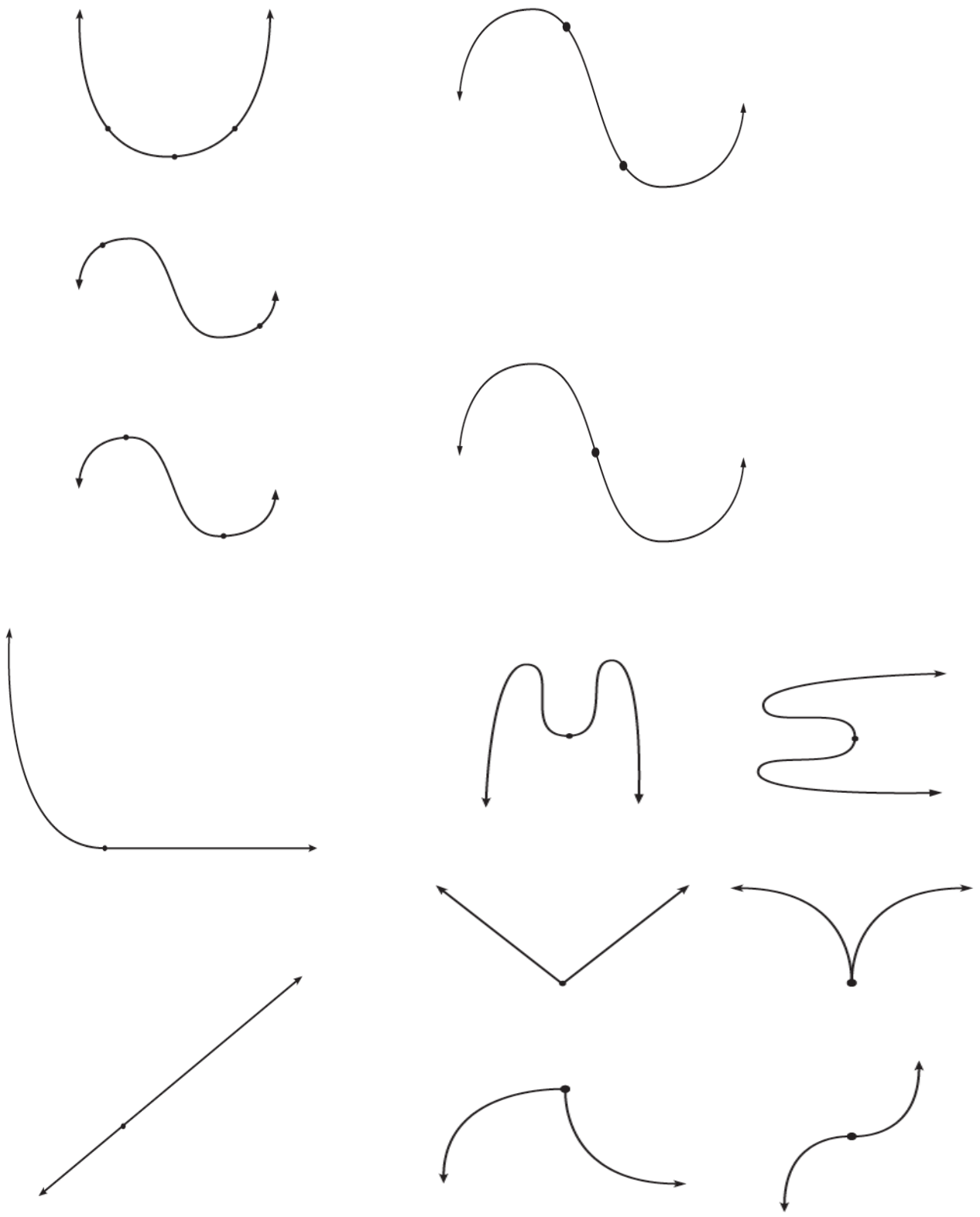
1.) What is a secant line?

2.) Describe the relationship, if any, between secant lines and tangent lines.

3.) Describe the relationship, if any, between the tangent function $(f(x)=\tan (x))$ and the tangent line? 


\section{Appendix C - Interview 2}

1. What is the definition of a tangent line?

2. Tangent line construction tasks- Six graphs.
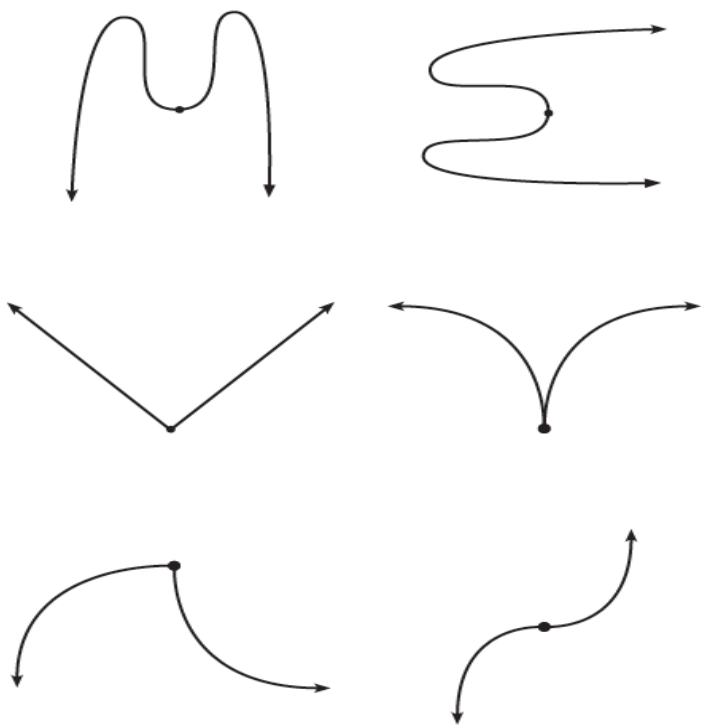

3. Sketch the graph of the derivative for the given function graph.

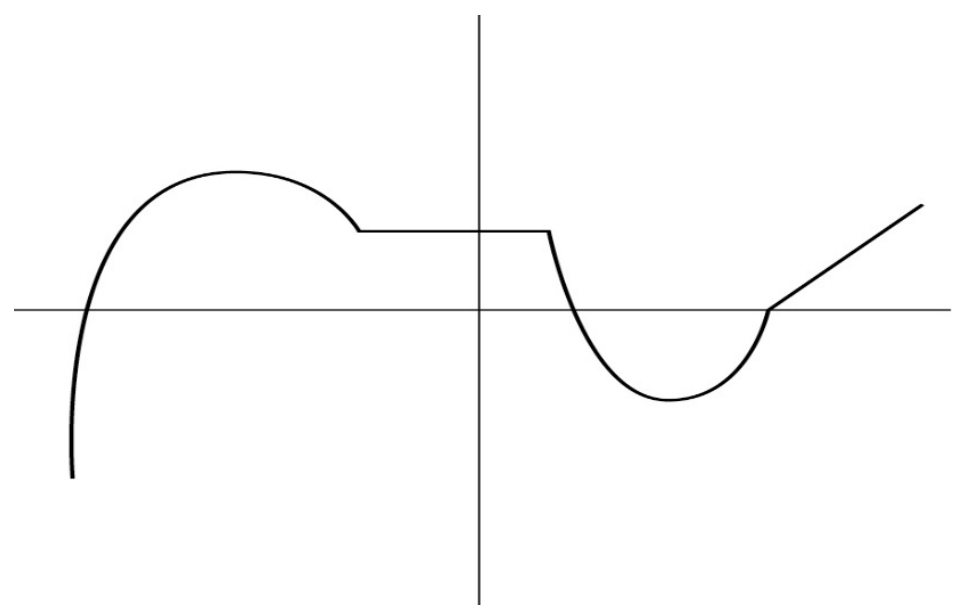




\section{APPENDIX D: Interview 3}

1. What is the definition of a tangent line?

2. Tangent line construction tasks- Six graphs.
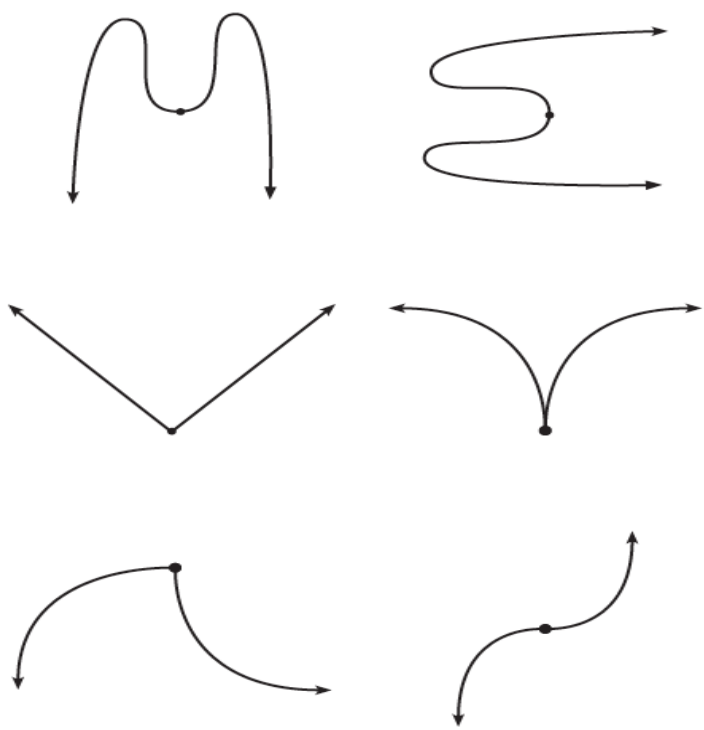

3. Sketch the graph of the derivative for the given function graph.

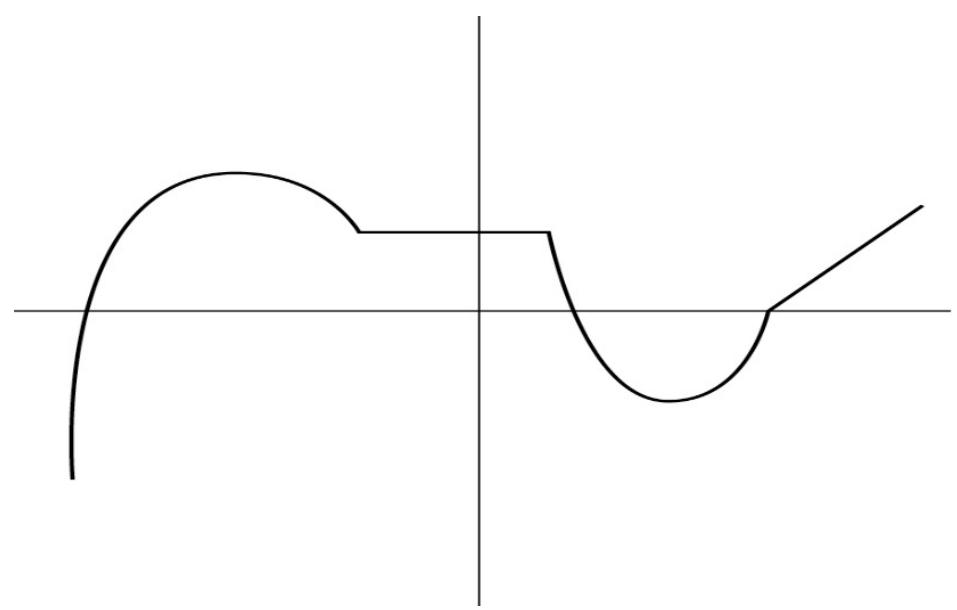

4. Graphically interpret, $\lim _{h \rightarrow 0} \frac{f(x+h)-f(x)}{h}$. 